\title{
Effect of Electrode Reversal on Induced Nonlinearity in Specialized Silicon Structures
}

\author{
by
}

Tahseen Haque, B.Eng. (Carleton) 2015

\begin{abstract}
A thesis submitted to the
Faculty of Graduate and Postdoctoral Affairs

in partial fulfillment of the requirements for the degree of
\end{abstract}

Master of Applied Science in Electrical Engineering

Ottawa-Carleton Institute for Electrical and Computer Engineering

Department of Electrical and Computer Engineering

Carleton University

Ottawa, Ontario

September, 2017

(C)Copyright

Tahseen Haque, 2017 


\section{Abstract}

Silica glass is a centrosymmetric material that does not support even-ordered nonlinearity. Despite the fact, its vast importance in photonic systems motivated researchers to develop techniques to break the centrosymmetric structure. However, to this day the effective nonlinearity in silica glass remains too low for practical applications in active nonlinear devices. In this study, a model of sub-dividing glass samples into multiple, alternating doped and undoped silica layers is investigated for its ability to enhance nonlinearity in silica glass. The glass samples are fabricated using a standard PECVD process. The dopant in the layers is germanium. Nonlinearity is induced in the samples using corona poling and the samples are characterized using Maker fringe measurements. The primary focus of this research is to analyze the distinctive properties of the nonlinearity profile that emerge due to certain alterations in the poling conditions. The following two steps introduce the alterations. In the first step, a set of two identical samples are poled with a positive voltage, but one of the samples is oriented in the opposite orientation inside the poling setup. One can alternatively think of it as a reversal of the electrodes. In the next step, another identical set of samples is poled in the same manner, but with a negative poling voltage. Thus, four glass samples are produced that possess unique nonlinearity profiles as shown by the Maker fringe measurements. It is observed that the intensity of SHG from the samples increases as the square of the intensity of the applied laser beam. The nonlinearity was stable and permanent for months. Different dopant types affect the Maker fringe patterns. A MATLAB hypothesis suggested that the modulation fringes for certain samples occur due to the formation of two nonlinear regions near the electrodes, each of width $5 \mu \mathrm{m}$. The samples which did not show modulation fringes only have a single nonlinear layer of width $9 \mu \mathrm{m}$. The findings of this research project can be implemented to understand the charge migration mechanism due to poling, thus enhancing the optimization capability of inducing nonlinearity in silica glass. 


\section{Acknowledgments}

I would like to first express my gratitude towards my supervisor, Professor Christopher Smelser for giving me the opportunity to be his student. I also sincerely appreciate the chance to work with Professor Jacques Albert. I feel fortunate to have played a small role in transforming their brilliant ideas into reality. Their help and kindness motivated me to work passionately for this project.

I am very grateful to Seyed Hamed Jafari for his immense help with my experiments and my understanding of all the "not so simple" concepts. And I must also mention that I thoroughly enjoyed working with my teammate Salah Abdullah.

My special thanks and admiration goes to Dr. Ksenia Yadav for her astounding work in Ph.D. research. Her Ph.D. thesis is a masterpiece which I found to be the best guidance out there for writing my own thesis. She set a standard that I hope to attain someday.

I would like to acknowledge the contributions of the Photonics and Laser technology department at Algonquin college. Their cooperation and patience with regards to our needs for research time and space in their facilities made this project possible.

I thank Professor Steve McGarry for allowing us to use his equipment for poling our samples. And I sincerely thank Nagui Mikhail and Albane Laronche for their support with lab apparatus and operations.

Thank you to all my dear friends at Carleton University, Dr. Mohamed Mohamed, Dr. Raisul Alam, Faisal, Sakib, Usama, Asif, Hassan and Saif, only to name a few, whose companionship rejuvenated me and helped me keep my head down till the finish line of graduate studies.

Lastly and most importantly, my sincere gratitude goes to my mother, father, and brother for their selfless help throughout my life. Their care and support are among the biggest reasons for all my success in life. 


\section{Table of Contents}

Abstract $\quad$ ii

Acknowledgments

Table of Contents $\quad$ iv

List of Figures $\quad$ vi

1 Introduction 1

1.1 Silica Glass as a Nonlinear Material . . . . . . . . . . . . . . . 3

1.1.1 Poling Methods . . . . . . . . . . . . . . . . . . 3

1.2 Limitations of Poled Silica . . . . . . . . . . . . . . . . 4

1.3 Charge Migration Model . . . . . . . . . . . . . . . . . . 5

1.3.1 Frozen-In Electric Field . . . . . . . . . . . . . . . . 7

1.3.2 Orientation of Hyper-Polarizable Entities . . . . . . . . . 9

1.4 General Equation to Describe Nonlinearity . . . . . . . . . . . . 9

1.5 Maker Fringe Measurements . . . . . . . . . . . . . . . . . 9

1.5.1 Coherence Length . . . . . . . . . . . . . . . . . . 10

1.5.2 Power of SH Signal . . . . . . . . . . . . . . . . 11

1.5.3 Maker Fringe Patterns . . . . . . . . . . . . . . . . . 14

1.6 Development of Multilayer Silica Structures . . . . . . . . . . . . 14

1.7 Poling of Single Stack Multilayer Structures with Alternate Voltages and Electrodes . . . . . . . . . . . . . . . . 19

1.8 Thesis Organization . . . . . . . . . . . . . . . . . . . . 20

2 Experimental Procedure $\quad 22$

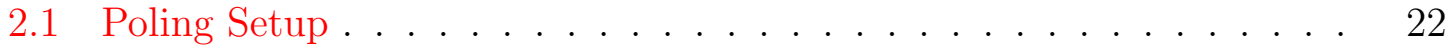


2.2 Maker Fringe Setup . . . . . . . . . . . . . . . . . . . . . . . . 24

2.3 Measurement Apparatus, Experimental Conditions, and Errors . . . . 26

2.3.1 Corona Poling . . . . . . . . . . . . . . . . . . . . 26

2.3.2 Maker Fringe Experiment . . . . . . . . . . . . . 27

3 Maker Fringe Characterization $\quad 29$

3.1 Maker Fringe Patterns . . . . . . . . . . . . . . . . . . . . . . . . 29

3.1 .1 Positive poling . . . . . . . . . . . . . . . . . . . . . 29

3.1 .2 Negative poling . . . . . . . . . . . . . . . . . . 31

3.2 Comparison of Maker Fringe Patterns . . . . . . . . . . . . . . 34

3.2.1 Similarity Between Maker Fringe Patterns . . . . . . . . . 36

3.2 .2 SH Peak Intensities . . . . . . . . . . . . . . . . . . 37

3.2.3 Reversal of Sample Orientation During Maker Fringe Experiment 38

3.3 Reproducibility of Results . . . . . . . . . . . . . . . . . . 42

3.3.1 Variation of Laser Beam Power . . . . . . . . . . . . . . 42

3.3.2 Effect of Aging on Induced Nonlinearity . . . . . . . . . . 44

3.3.3 Phosphorous Doped Multilayer Structure . . . . . . . . . . . 45

4 Matlab Simulation $\quad 50$

4.1 Numerical Estimate of Induced Nonlinearity . . . . . . . . . . . 51

4.1.1 Maker Fringe Pattern with Modulation Fringes . . . . . . . 51

4.1.2 Maker Fringe Pattern without Modulation Fringes . . . . . . 51

5 Summary and Future Work $\quad 56$

5.1 Recommendations of Future Work . . . . . . . . . . . . . 57

$\begin{array}{ll}\text { List of References } & 59\end{array}$ 


\section{List of Figures}

1.1 Setup diagrams for the most popular poling methods. . . . . . . . . 4

1.2 Silica glass matrix before poling. . . . . . . . . . . . . . 6

1.3 Charge migration model for the poling-induced second-order nonlinearity in glasses (assuming a non-blocking anode electrode) . . . . . 7

1.4 Charge migration model for a blocking anode condition . . . . . . 8

1.5 Power conversion between fundamental and SHG waves in a nonlinear medium. . . . . . . . . . . . . . . . . . 10

1.6 Propagation of fundamental wave and the corresponding second harmonic wave, through a nonlinear medium . . . . . . . . . . . . 11

1.7 Maker fringe patterns of different types of nonlinearity profile . . . 15

1.8 Waveguide layers fabricated by Arentoft et al. . . . . . . . . . . 16

1.9 Thin film multilayer silica structure developed by Fleming et al. . . . 17

1.10 Thin film multilayer silica structure with alternating doped layers, developed by Yadav et al. . . . . . . . . . . . . . . . . 18

1.11 Multilayer silica structure with forty alternating doped layers, developed by Yadav et al. . . . . . . . . . . . . . . . . . . . 19

1.12 Progression diagram showing the various stages in this research project 21

2.1 Thin film samples with 8 alternating layers . . . . . . . . . . . 23

2.2 Setup diagrams for poling voltage and orientation. . . . . . . . . 25

2.3 Setup for Maker Fringe Experiments . . . . . . . . . . . . . 26

2.4 Maker fringe pattern obtained from a Quartz sample . . . . . . 28

3.1 Maker fringe pattern for SPbulk. . . . . . . . . . . . . 30

3.2 Maker fringe pattern for SPup. . . . . . . . . . . . . . 31

3.3 Maker fringe pattern for SPdown. . . . . . . . . . . . . . . 32

3.4 Maker fringe pattern for SNbulk. . . . . . . . . . . . . . 33

3.5 Maker fringe pattern for SNup. . . . . . . . . . . . . 33 
3.6 Maker fringe pattern for SNdown. . . . . . . . . . . . . . . . 34

3.7 Charge migration model of the poled samples . . . . . . . . . . 35

3.8 Comparison of Maker fringe patterns of SPup and SNdown . . . . . 36

3.9 Comparison of Maker fringe patterns of SPdown and SNup . . . . . 37

3.10 Comparison between the SH intensities of the maker fringe patterns of SPup, SPdown and SPbulk . . . . . . . . . . . . . 38

3.11 Comparison between the SH intensities of the maker fringe patterns of SNup, SNdown and SNbulk . . . . . . . . . . . . . . . 39

3.12 Alternate orientations of the samples during Maker fringe characteri-

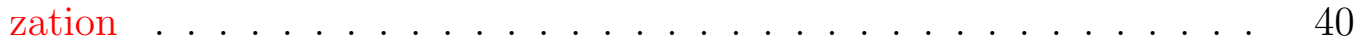

3.13 Comparisons between Maker fringe pattern from the two faces of SPup. 40

3.14 Comparisons between Maker fringe pattern from the two faces of SPdown. 41

3.15 Comparisons between Maker fringe pattern from the two faces of SNup. 41

3.16 Comparisons between Maker fringe pattern from the two faces of SNdown. . . . . . . . . . . . . . . . . . 42

3.17 Graph showing the applicability of the relationship $I_{2 \omega} \propto I_{\omega}^{2}$ for the multilayer structures . . . . . . . . . . . . . . . . . 44 43

3.18 Comparison between Maker fringe patterns from repeated measurements of sample SPup, input power $<1 \mathrm{~mW} \ldots \ldots \ldots$. . . . . . 45

3.19 Comparison between Maker fringe patterns from experiments 12 days apart (sample SPup). . . . . . . . . . . . . . . . . 46

3.20 Comparison between Maker fringe patterns from experiments 7 days apart (sample SNdown). . . . . . . . . . . . . . . . . . 47

3.21 Maker fringe patterns from positively poled phosphorous doped sample. 47

3.22 Maker fringe patterns from negatively poled phosphorous doped sample. 48

$3.23 \mathrm{P}_{2} \mathrm{O}_{5}$ formation in silica matrix $\ldots \ldots \ldots \ldots \ldots$

4.1 MATLAB simulation vs. experimental Maker fringe patterns for samples SPup and SNdown . . . . . . . . . . . . .

4.2 MATLAB simulation vs. experimental Maker fringe patterns for samples SPdown and SNup . . . . . . . . . . . . . . . .

4.3 Simulated charge migration model of the poled samples. The labels indicate the extent and depth of the induced nonlinearity . . . . . . . 


\section{Chapter 1}

\section{Introduction}

The role of photonics has become increasingly important in enhancing the technological capabilities in today's world. Through intense research and development, scientists are producing new possibilities in the areas of communications, life sciences, healthcare, security and safety, imaging, broadband internet, and manufacturing, among many others. In fact, in telecommunications, optical communications systems have superseded traditional radio systems in the form of fiber-optic communications.

The field of photonics was revolutionized by the invention of lasers in 1960. Lasers helped produce unique optical phenomena by generating light at very high intensities. Theories of linear optics dominated the pre-laser era. In linear optics, superposition

of light applies, optical properties of materials are independent of light intensity, the frequency of light is independent of the medium of travel, and light cannot be used to control light in a region. The revolutionary discovery of non-linear optics revealed that at very high intensities we could, in fact, manipulate light in ways that were perceived to be impossible.

Silica glass plays a vital role for commercial applications in the field of photonics. Its applications range from chip fabrication to optical communication systems that span continents. Besides its low cost and wide availability, its exceptional physiochemical properties proved to be remarkably advantageous. For example, it is immune to electromagnetic interference and supports high transmittance for various wavelengths, including infrared radiation. This property is suitable for long distance communications. It has a very high optical damage threshold, making it excellent for applications with high powered lasers. It exhibits low thermal expansion and can be formed into many desired shapes, sizes or thicknesses with a broad range of 
fabrication processes, thus making its production and implementation relatively easy. Silica's amorphous structure results in it not being birefringent [1].

On account of its amorphous structure, silica is also centrosymmetric. This means that the crystal structure has a center through which it displays inversion symmetry. Consequently, silica does not possess an even ordered nonlinearity. This property limits silica from exhibiting the second-order nonlinear optical process, one of which is second harmonic generation (SHG). The SHG technique has found several key applications today, in areas such as laser frequency conversion (frequency doubling), nonlinear spectroscopy, quantum cryptography [2], surface study and characterization of second order nonlinear optical (NLO) properties of materials [3].

At present, many NLO applications use crystalline dielectric materials, such as Lithium Niobate $\left(\mathrm{LiNbO}_{3}\right.$ ), Potassium Titanyl Phosphate (KTP) and Potassium Titanyl Phosphate (KDP). The molecular structure of the crystals lacks a center of symmetry or inversion symmetry, thus inherently exhibiting nonlinear optical effects. Besides a high conversion efficiency for SHG, the crystals are stable at room temperature and relatively easy to manufacture into different sample structures.

There are still some properties that cause drawbacks for a major group of NLO applications. $\mathrm{LiNbO}_{3}$ crystals are prone to the effect of optical damage. Optical damage is a semi-permanent alteration in the refractive index due to the intense light passing through the sample. The affected region gives rise to beam distortion. Industrial applications introduce dopants such as Magnesium and Zinc to improve the optical damage threshold of these crystals [4]. Another significant disadvantage arises from the lack of compatibility with the existing silica based infrastructure. $\mathrm{LiNbO}_{3}$ and other crystalline materials cannot be made into fibers. There are also significant differences in the optical and thermal properties when compared to silica. Therefore, manufacturers need to implement complicated designs, to adapt and minimize losses in the systems during integration. These issues add to the cost of today's high-speed optical networks.

Ongoing research explores appropriate modifications, perhaps even replacements of the nonlinear materials in conventional optical systems. The 1970s and '80s saw substantial research on the nonlinear interaction effects in glass optical fibers. In 1986, Margulis et al. reported efficient SHG coming from a Ge-doped glass fiber [5]. This discovery triggered a spark of interest within the field of Photonics, because of the prospects of eliminating most losses and integration difficulties by building active 
nonlinear devices with only one material, silica.

\subsection{Silica Glass as a Nonlinear Material}

The second order susceptibility tensor of silica, $\chi^{(2)}=0$, depicting the inversion symmetry of the molecular structure and the lack of any nonlinear optical properties. Its lowest nonlinear coefficient arises from the third order susceptibility tensor, $\chi^{(3)}$. Despite the fact, Margulis et al. reported SHG in silica-based optical fiber, which meant a non-zero $\chi^{(2)}$ of silica glass. In the experiment, they coupled a high-intensity pulsed beam into a Ge-doped silica-based optical fiber and observed frequency doubling after several hours of constant illumination. The magnitude of the output steadily increased with time. After about 12 hours, the nonlinear process finally saturated. The average power was measured to be $0.7 \mathrm{~mW}$, with a peak power as high as $1.6 \mathrm{~mW}$. This experimental procedure is now called optical poling or photoinduced SHG process. The phenomenon is understood to be a consequence of poling of the fiber to break its symmetry.

\subsubsection{Poling Methods}

Researchers developed new ways of poling, to raise the amount of induced secondorder nonlinearity (SON) in silica glass. This section contains a summary of poling methods developed over the years.

\section{Thermal Poling}

In 1991, Myers et al. developed the thermal poling process [6]. As shown in Figure 1.1, a silica glass sample is heated with an ambient temperature of 250 to $325{ }^{\circ} \mathrm{C}$ while applying a constant voltage of 3 to $5 \mathrm{kV}$ across it. After 5 to 30 minutes, the heating is turned off to allow the sample to cool down to room temperature. The electric field must be in place until the sample reaches room temperature. Characterization techniques such as Maker fringes, second harmonic (SH) microscopy, layer peeling (LP) and etching are used to characterize induced SON [7] [8]. The magnitude of the induced SON coefficient was measured to be $\sim 1 \mathrm{pm} / \mathrm{V}$. It is apparent that the SON coefficient of silica glass is much lower than the $81 \mathrm{pm} / \mathrm{V}$ obtained from $\mathrm{LiNbO}_{3}$ 
crystals. Nonetheless, scientists were enthusiastic to find out more about the underlying mechanism for the cause of induced SON in silica glass. Section 1.3 discusses the two possible mechanisms of modification of the silica glass structure that occur during the poling process.

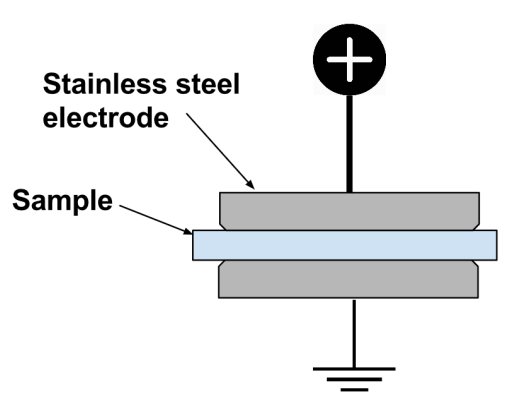

(a) A thermal poling setup.
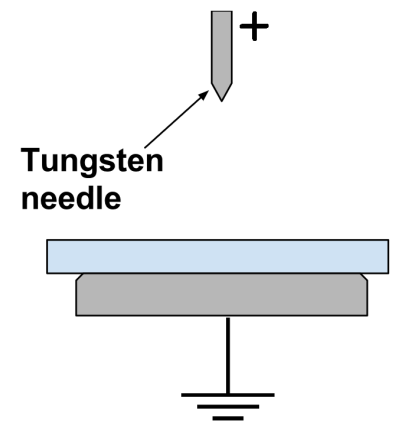

(b) A corona poling setup.

Figure 1.1: Setup diagrams for the most popular poling methods.

\section{Corona Poling}

Another method of inducing nonlinearity in silica glass is the corona poling method. It is a variation of thermal poling, where the top electrode is replaced with a tungsten needle $\sim 1 \mathrm{~cm}$ above the sample, as shown in Figure 1.1 [9].

Researchers developed many more poling methods aiming to enhance the induced SON, such as $\mathrm{CO}_{2}$ laser assisted poling [10], UV poling [11], UV-fs poling [12], electron-beam poling [13] and proton implantation poling [14].

\section{$1.2 \quad$ Limitations of Poled Silica}

The poling methods discussed in 1.1 .1 produced SON only comparable to that of thermal poling. The magnitude of the effective nonlinearity still fell very short of practical requirements. The most successful experiment used germanosilicate thin films and produced a SON coefficient of only $1.6 \mathrm{pm} / \mathrm{V}$ [15]. Although this value is much lower than the strongest SON tensor element of $\mathrm{LiNbO}_{3}\left(\chi_{33}^{(2)}=81 \mathrm{pm} / \mathrm{V}\right)$, it is comparable to the $\chi^{(2)}$ values obtained from other nonlinear materials such as KDP and quartz. Therefore, poled silica can achieve reasonable levels of frequency doubling 
in industrial applications, especially with devices that support long interaction lengths (e.g. silica waveguide or fiber based devices).

A more significant issue arises due to the small extent of the generated nonlinear region in silica glass (discussed in section 1.3) [6], resulting in incompatibility with the conventional waveguide and fiber dimensions. The overlap between the propagating optical wave and the nonlinear region is far too small for sufficient nonlinear interaction [16]. An adequate measure of second harmonic generation would require impractically long device lengths. One should note that glasses with low impurity content such as Suprasil, sustains nonlinear regions spanning tens of micrometers. However, the effective $\chi^{(2)}$ also falls off by an order of magnitude counteracting the gain in this case.

Poling at a greater temperature or electric field, or for a longer duration can not improve the induced nonlinearity. During the poling process, a positively charged layer forms at the anode (discussed in section 1.3). Raising the parameters of poling conditions, cause the positive charges to migrate into the bulk of the sample. The charges combine with the negatively charged non-bridging oxygen ions, which initially formed due to the depletion of alkali ions. This a detrimental process through which the depletion region is reduced in size, thus reducing the effective SON of silica glass as well.

Another issue with silica glass is the sensitivity of the induced SON to the variability of the concentration of impurity content, $N_{0}$. Impurity content is low in silica glass (e.g. $0.01 \mathrm{ppm}$ for glass manufactured by Heraeus). At these low levels, impurity content typically vary significantly (e.g. $0.04 \mathrm{ppm}$ is still low, but it is four times $0.01 \mathrm{ppm})$. Since the nonlinear layer width, $w$ is inversely proportional to $\sqrt{N_{0}}$, a four-fold increase in $N_{0}$ results in $w$ to being reduced by a factor of two [17]. Hence, it is difficult to control the extent of nonlinearity and the magnitude of the $\chi^{(2)}$ in silica glass.

\subsection{Charge Migration Model}

It is worthwhile to investigate the underlying mechanisms taking place for inducing SON in silica glass. This section will present the work of several research groups that came up with models of charge migration. Various studies offered different explanations in their models, thus instigating much debate in the field. 
To begin with, we consider the molecular structure of silica glass, as shown in Figure 1.2. It consists of silicon ions that are the network formers. It has oxygen ions of two types. When two tetrahedron shares one oxygen ion between themselves, it is called a bridging oxygen. On the other hand, when a oxygen ion bonds to only one silicon ion, it is referred to as a non-bridging oxygen. The non-bridging oxygen ions have a net negative charge of 1-. The modifier ions, also called impurities, are alkali or alkaline earth metal ions such as $\mathrm{Na}^{+}, \mathrm{Li}^{+}$and $\mathrm{Ca}^{+}$. These ions have a net positive charge of $1+$.

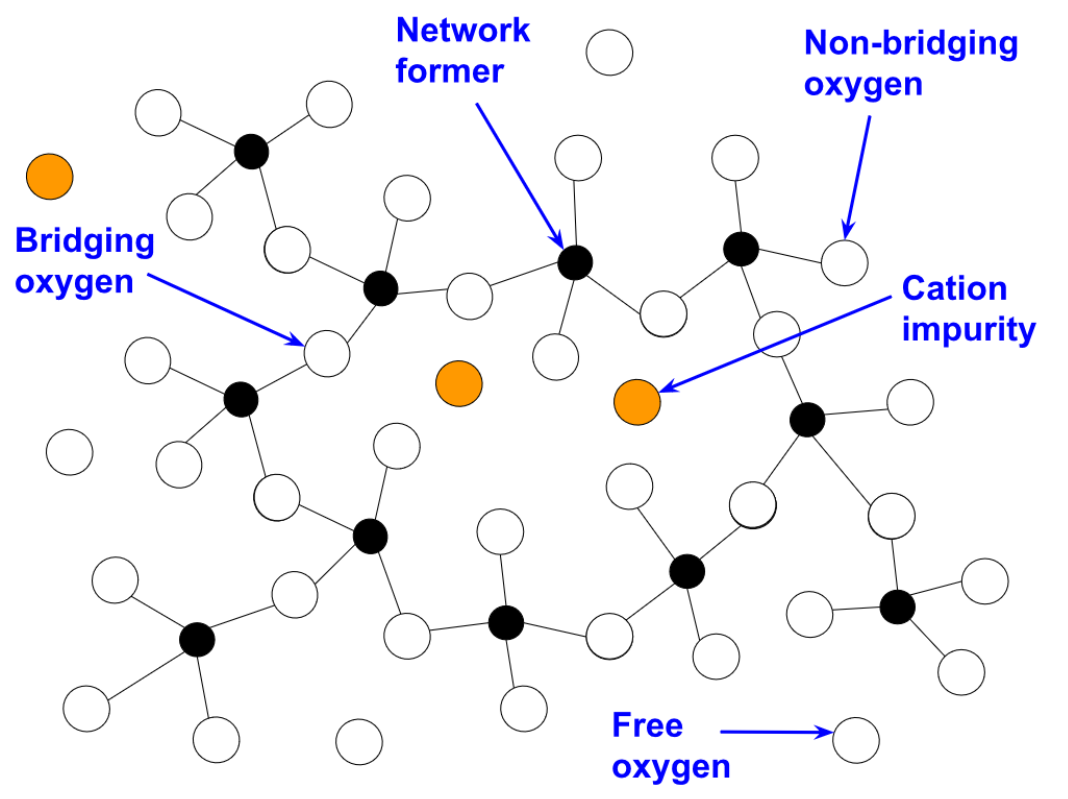

Figure 1.2: Silica glass matrix before poling.

Non-linearity in silica glass is said to occur due to an electric field-induced ion migration [18]. During the poling process, the elevated temperature causes the ions to be more mobile. Under the applied external electric field, the most mobile alkali ions or cations, migrate away from the anode, and are neutralized by incoming electrons at the cathode [19]. The migrating cations leave behind the negatively charged nonbridging oxygen ions. Thus, a negative space-charge forms in the anode region, with a spatial extent of 2 to $14 \mu \mathrm{m}$ beneath the anode [6] [20] [21]. Beyond this point, experimental evidence suggests two possible mechanisms of charge migration; Frozenin electric field and orientation of hyper-polarizable entities. The following sections describe the two phenomena. 


\subsubsection{Frozen-In Electric Field}

This model considers two cases of poling conditions; nonblocking electrode and blocking electrode.

\section{Nonblocking Electrode}

In this case, where the anode is free to supply cations, a positive charge layer forms at the anode-glass surface, as shown in 1.3. The positive charges are frequently considered to be $\mathrm{H}_{3} \mathrm{O}^{+}$or $\mathrm{H}^{+}$ions, supplied by air. Alternatively, the formation of this layer may also be due to the emission of electrons from the silica glass sample to the anode [22]. Nevertheless, the applied external field mainly drops across the depleted region and ultimately contributes in inducing SON in silica glass.

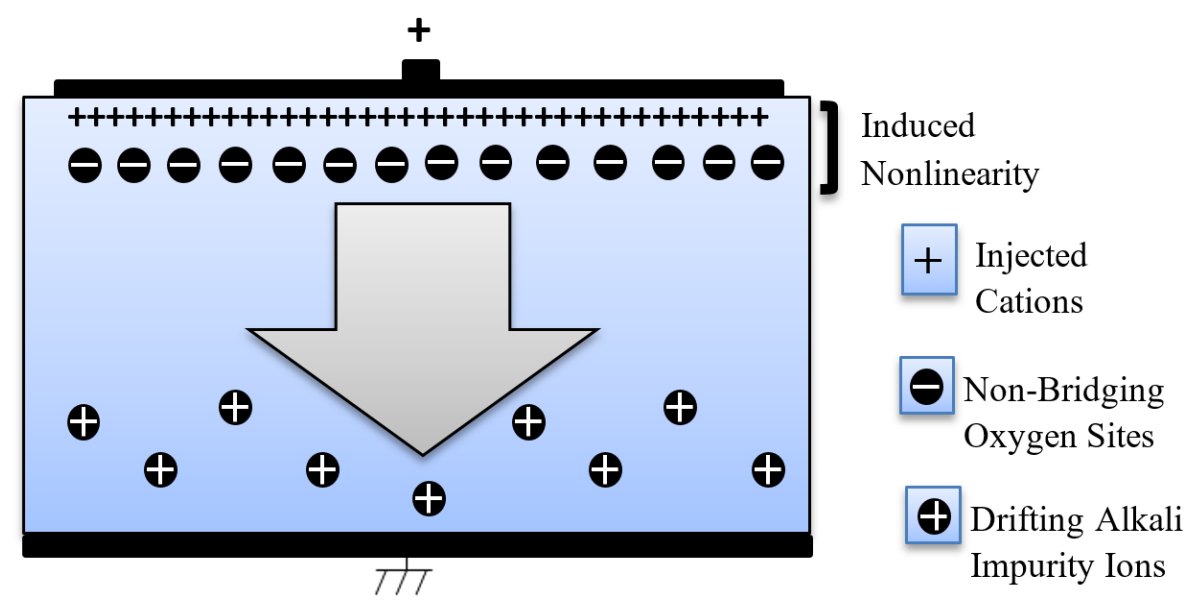

Figure 1.3: Charge migration model for the poling-induced second-order nonlinearity in glasses (assuming a non-blocking anode electrode)

\section{Blocking Electrode}

In the case of a blocking anode, where the supply of cations is restricted, experimental evidence suggest that positive charges still compensate the negative space-charge region, indicating that some form of ionization due to the high electric field takes place. However, the mechanism of charge compensation for this phenomenon is not clear [21]. The "anodic bonding community" believes that the high temperature and electric field conditions, aid in the breaking of the bonds between the nonbridging 
oxygen ions and the respective tetrahedra (silicon ion). The oxygen ions with net charge 1- tend to travel towards the anode, leaving behind silicon sites with valence 3+, like shown in Figure 1.4 [23]. The applied external field mainly drops across the depleted region. On the other hand, the "glass bonding community" believes that the activation energy of the nonbridging oxygen ions is too high, restricting them of any movement. Rather, with appropriate structural rearrangements, electrons are released, which hop over towards the anode through the nonbridging oxygen sites [24], forming a positive space-charge within the depletion region. Nearly all of the applied electric field drops across this depletion region, which ultimately contributes in inducing SON.

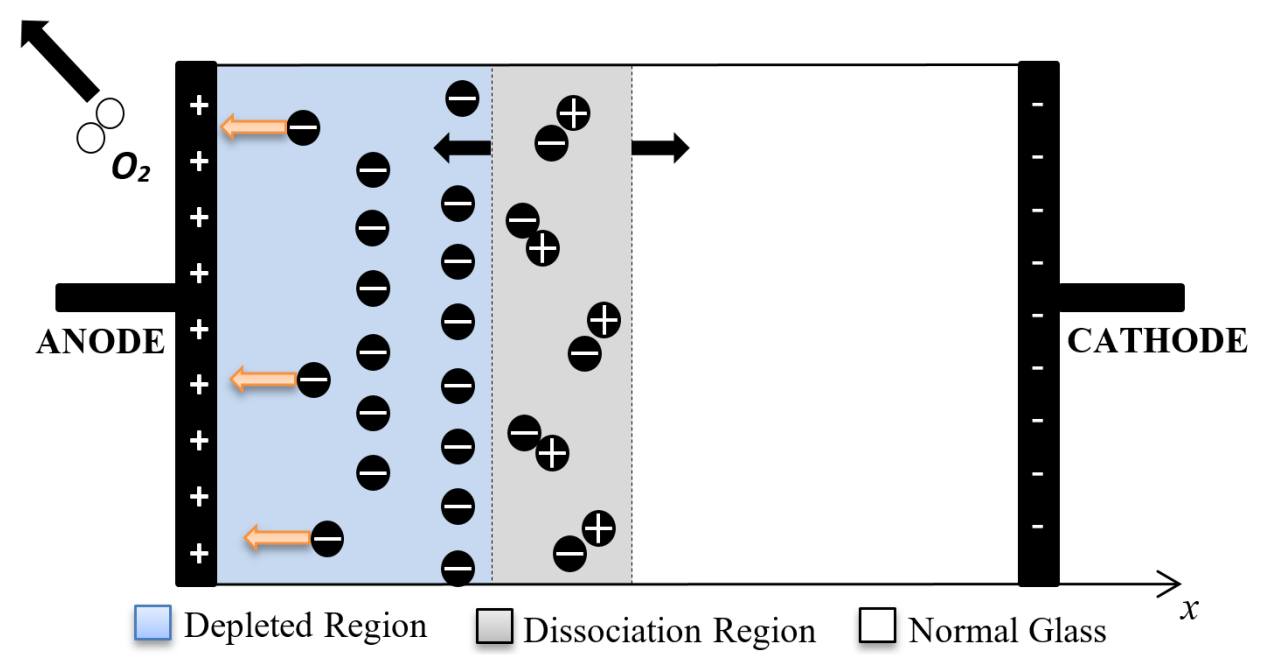

Figure 1.4: Charge migration model for a blocking anode condition

Similar events may be occurring on the side of the cathode, due to the accumulation of cations that happened at the beginning of the poling process.

Regardless of the poling conditions, when the sample is cooled down to room temperature, the ions are frozen in place, creating a strong frozen in an electric field. This electric field couples with the third-order nonlinearity of glass, to produce an effective second-order susceptibility, $\chi^{(2)}$. In this case, $\chi^{(2)}$ is proportional to $\chi^{(3)} E_{d c}[19]$. 


\subsubsection{Orientation of Hyper-Polarizable Entities}

The amorphous form of the silica glass structure supports many different bond configurations with the same atomic configuration. Therefore, in the second model to describe the induced nonlinearity, the elevated temperature and high electric field are said to cause hyperpolarizable entities (such as a bond, dipole, defect or nanocrystalline) to orient in the direction of the applied field [25] [19]. When the sample cools down to room temperature, the dipoles remain frozen in their new orientation, thus breaking the symmetry of the glass and inducing SON.

\subsection{General Equation to Describe Nonlinearity}

The general expression for the effective second order susceptibility tensor, consisting of the effects derived from both mechanisms discussed above, is as follows [25],

$$
\chi_{e f f}^{(2)}=3 \chi^{(3)} E_{d c}+\frac{N p \beta}{5 k T} E_{d c}
$$

The first term describes the interaction between the frozen-in electric field, $E_{d c}$, and the third order susceptibility tensor of silica glass, $\chi^{(3)}$. The second term describes the electric-field-induced orientation of the molecular second-order hyperpolarizability, $\beta . p$ is the permanent dipole moment associated with the bond, $k$ is the Boltzmann constant, $T$ is the temperature in Kelvins, and $N$ is the number of molecules.

Over the course of time, more experimental evidence supported the theory of frozen-in electric field as the reason for SON in silica glass. Researchers confirmed that it is, in fact, alkali ions that migrate towards the cathode to form the depletion region [26], resulting in a charge separation and thus a large electric field [27]. The positive charge layer at the anode-glass interface was also detected using techniques such as laser induced pressure pulse probe and noncollinear frequency conversion of two femtosecond pulses [22] [28]. Kazansky et al. demonstrated that the $\chi^{(2)}$ tensor symmetry is consistent with that of $\chi^{(3)}$ in silica, confirming that $\chi^{(2)}$ does originate from a $\chi^{(3)}$ process [29] like shown in equation (1.1).

\subsection{Maker Fringe Measurements}

This section presents the theory of Maker fringe measurements in brief. 


\subsubsection{Coherence Length}

When a wave with a fundamental frequency travels through a dispersive nonlinear region, it generates second harmonic (SH) waves. Due to their different phase velocities, the fundamental and the harmonic waves accumulate a $\pi$ phase shift over an interval of length. Over that interval, the generated $\mathrm{SH}$ waves along the path constructively interfere with each other to rise to a peak intensity. In the next interval, the $\mathrm{SH}$ waves destructively interfere, in which case the power flows back to the fundamental wave. For this study, the periodicity of this effect is taken to be the coherence length, $L_{c}$. This characteristic is depicted with a simple sine wave in Figure 1.5. In this figure, the red line represents the interval in which the fundamental and the generated SH waves go out of phase. The corresponding interval of length is $L_{c}$.

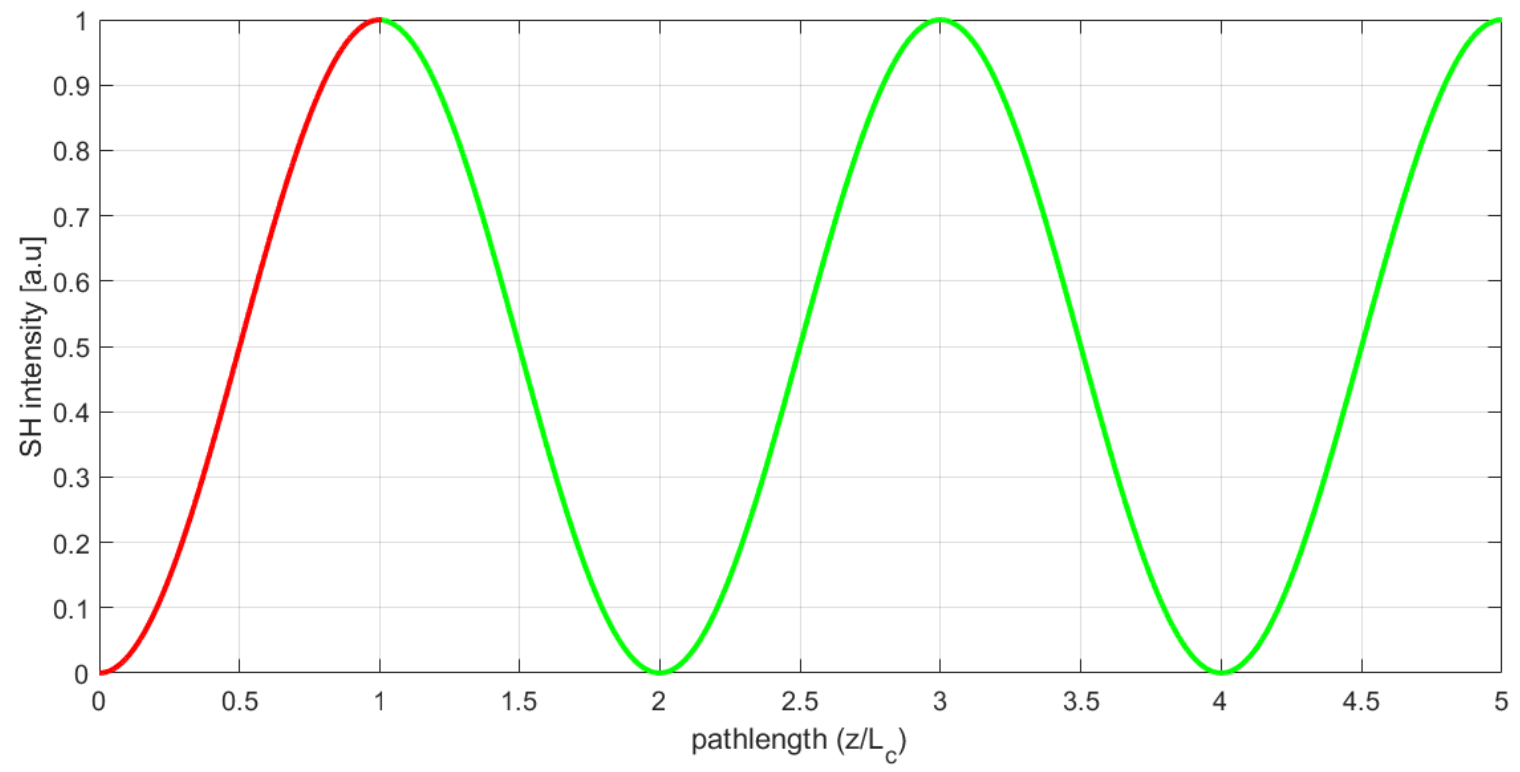

Figure 1.5: Power conversion between fundamental and SHG waves in a nonlinear medium.

$L_{c}$ can be expressed as a function of fundamental wavelength and the refractive indices of the two waves as follows [30],

$$
L_{c}=\frac{\lambda_{\omega}}{4\left|n_{2 \omega}-n_{\omega}\right|}
$$




\subsubsection{Power of SH Signal}

Maker fringe measurement is a technique to determine the nonlinear susceptibility of a material by comparing the $\mathrm{SH}$ signal generated by the material to the signal from a previously known standard (e.g. $\mathrm{LiNbO}_{3}$ and crystalline quartz) [31] [32]. Figure 1.6 shows a simplified ray diagram depicting a setup for Maker fringe measurements. The laser beam goes through a sample as it rotates about the y-axis. The rotation of the sample causes the path length, which is essentially the length over which the fundamental wave interacts with the material, to vary. The angledependent nonlinear path length is $L / \cos \theta_{2 \omega}$, where $L$ is the width of the sample. In this research project, $\theta_{\omega} \approx \theta_{2 \omega}$ is assumed for the purpose of simplification. This assumption is justified because the samples under investigation are based on silica. Silica glass exhibits high transmittance and very low dispersion.

When the path length is larger than the coherence length of the material, the intensity of the SHG varies periodically (as discussed in 1.5.1). Thus, the Maker fringe patterns produce fringes or periodic oscillations in the measured SH power.

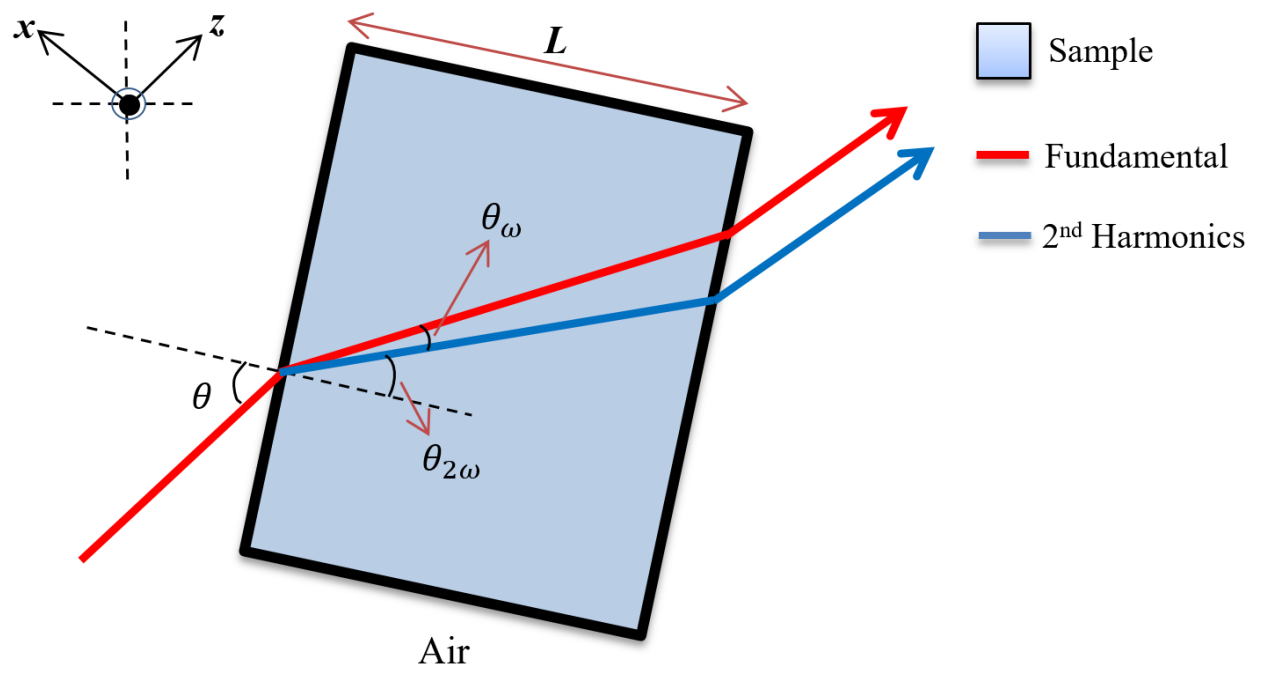

Figure 1.6: Propagation of fundamental wave and the corresponding second harmonic wave, through a nonlinear medium

During Maker fringe measurements, the refraction of the fundamental and the $\mathrm{SH}$ waves at the interfaces (between air- sample, and sample-air) affects the power of the $\mathrm{SH}$ signal. This effect can be determined using Fresnel transmission equations. The 
derivation of an expression for the $\mathrm{SH}$ power is shown below.

In this project, only p-polarized (horizontally polarized) beam is used to characterize the samples. So, the derivation does not include Fresnel equations corresponding to s-polarization.

The power transmittance of the fundamental beam in the air-sample interface is given by:

$$
T_{\omega}=\frac{n_{\omega} \cos \theta_{\omega}}{\cos \theta}\left|t_{p}^{a \rightarrow s}\right|^{2}
$$

where $\omega$ is the angular frequency of the fundamental wave, $n_{\omega}$ is the refractive index of the medium at the fundamental wavelength, and $t_{p}^{a \rightarrow s}$ is the Fresnel transmission term for the air-sample interface, expressed as:

$$
t_{p}^{a \rightarrow s}=\frac{2 \cos \theta}{\cos \theta_{\omega}+n_{\omega} \cos \theta}
$$

Similarly, the power transmittance of the $\mathrm{SH}$ wave at the sample-air interface is:

$$
T_{2 \omega}=\frac{\cos \theta}{n_{2 \omega} \cos \theta_{2 \omega}}\left|t_{p}^{s \rightarrow a}\right|^{2}
$$

where $n_{2 \omega}$ is the refractive index of the medium at the SH wavelength, and $t_{p}^{s \rightarrow a}$ is the Fresnel transmission term given by:

$$
t_{p}^{s \rightarrow a}=\frac{2 n_{2 \omega} \cos \theta_{2 \omega}}{\cos \theta_{2 \omega}+n_{2 \omega} \cos \theta}
$$

The SH generated from a slab with uniform nonlinearity and thickness $L$, can be calculated using the equation [33]:

$$
E_{2 \omega}(z)=\epsilon_{0} \frac{-j \omega}{2} \sqrt{\frac{\mu_{0}}{\epsilon}} E_{\omega}^{2} \chi_{e f f} \frac{e^{j \Delta k L-1}}{j \Delta k}
$$

Since the intensity of a monochromatic wave is [34]: 


$$
I=\frac{|E|^{2}}{2 \eta}
$$

where $\eta$ is the impedance of the medium, given by:

$$
\eta=\sqrt{\frac{\mu_{0}}{\epsilon}}=\frac{1}{n_{2 \omega} \epsilon_{0} c}
$$

using equations 1.7, 1.8 and 1.9, the second harmonic intensity is derived to be:

$$
I_{2 \omega}=\epsilon_{0}^{2} \frac{\omega^{2} L^{2}}{2 n_{\omega}^{2} n_{2 \omega}}\left(\frac{\mu_{0}}{\epsilon_{0}}\right)^{\frac{3}{2}} \chi_{\text {eff }}^{2} I_{\omega}^{2}\left[\frac{\sin \left(\frac{\Delta k L}{2}\right)}{\frac{\Delta k L}{2}}\right]^{2}
$$

which can also be written as:

$$
I_{2 \omega}=\frac{\omega^{2} L^{2}}{2 \epsilon_{0} c^{3} n_{\omega}^{2} n_{2 \omega}} \chi_{e f f}^{2} I_{\omega}^{2}\left[\frac{\sin \left(\frac{\Delta k L}{2}\right)}{\frac{\Delta k L}{2}}\right]^{2}
$$

Finally, the power of the SH signal, which is linearly proportional to its intensity, is derived to be:

$$
P_{2 \omega}=\frac{\omega^{2} L^{2}}{2 \epsilon_{0} c^{3} n_{\omega}^{2} n_{2 \omega}} B(\theta) \chi_{e f f}^{2}(\theta) \frac{P_{\omega}^{2}}{A}\left[\frac{\sin \left(\frac{\Delta k L}{2}\right)}{\frac{\Delta k L}{2}}\right]^{2} T_{\omega}^{2}(\theta) T_{2 \omega}(\theta)
$$

here $B$ is the beam correction factor, and $A$ is the cross-sectional area of the fundamental wave before entering the sample. The beam correction factor in included to counteract the change in the diameter of the beam inside the sample due to refraction at the air-sample interface. The derived equation (1.12), describes the power of the $\mathrm{SH}$ signal measured in Maker fringe experiments. It can be applied to calculate the $\mathrm{SH}$ power of a sample of interest and compared with a known standard to obtain its nonlinear susceptibility tensor. 


\subsubsection{Maker Fringe Patterns}

During the Maker fringe characterization of a nonlinear sample, a bulk nonlinearity profile (that of a nonlinear crystal as an example) would produce modulation fringes, like shown in Figure 1.7a. This behavior is characteristic of a nonlinear region width greater than $L_{c}$. In every cycle of $\pi$ phase shift accumulated between the fundamental and the generated SH waves, constructive (or destructive) interferences occurs, thus producing modulation fringes in the Maker fringe pattern.

On the other hand, a sample with a thin nonlinearity (e.g. poled silica) will produce a Maker fringe pattern like that shown in Figure 1.7b. No modulation fringes appear in this case. The generated SH waves exit the nonlinear region before encountering interferences down the path of travel because the nonlinearity width is less than $L_{c}$.

Modulation of fringes in a Maker fringe pattern can also occur due to the existence of two separate nonlinear regions in a sample, each of width less than $L_{c}$. Figure $1.7 \mathrm{c}$ a Maker fringe pattern generated from two thin layers of nonlinearity located on either side of a sample. In this case, the generated SH waves from the two regions interact to cause interferences, hence the modulation fringes. Note the similarity to the pattern observed for bulk nonlinearity (in Figure 1.7a).

The different types of nonlinearity profiles discussed in this section are used to analyze the results in chapter 3 .

\subsection{Development of Multilayer Silica Structures}

In 2001, Arentoft et al. reported a sharp rise in nonlinearity induced in a thermally poled silica waveguide [35]. As shown in Figure 1.8, the core layer consisted of germanium-doped silicon oxy-nitride (Ge:SiON) and the cladding layers of pure silicon dioxide $\left(\mathrm{SiO}_{2}\right)$. A layer of silver-containing paint and silicon wafer was used as the top and bottom electrodes, respectively. Two different samples were poled, one with positive and another with negative voltages. While the negatively poled sample showed negligible SHG, the positively poled sample possessed significant SHG. The nonlinear susceptibility coefficient, $\chi_{33}^{(2)}$ was calculated to be $22 \mathrm{pm} / \mathrm{V}$. Secondary ion mass spectrometry (SIMS) measurements showed peaks in concentration depth 


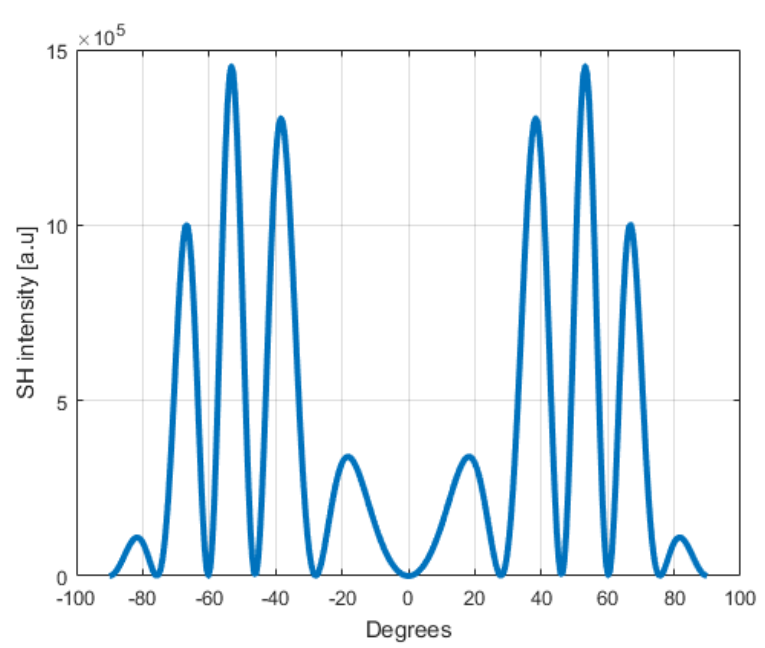

(a) Bulk nonlinearity

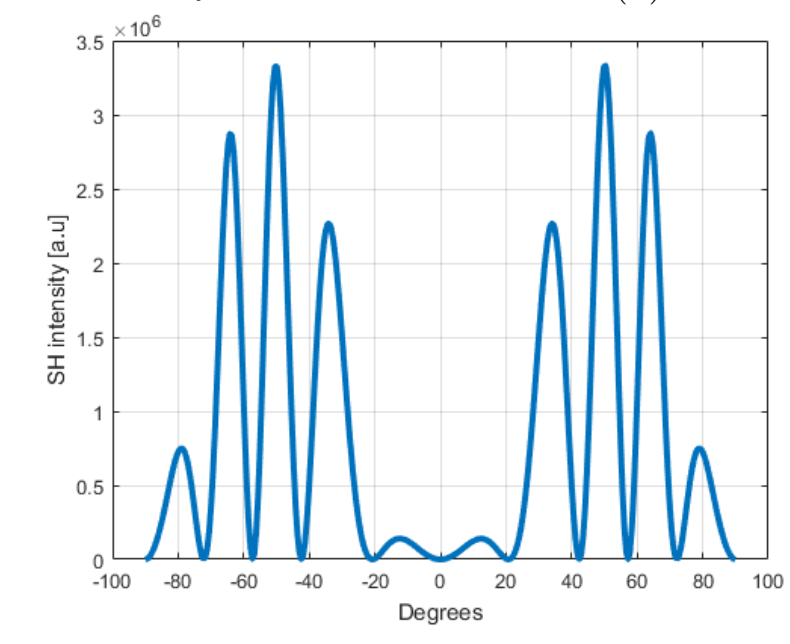

(c) Two thin nonlinearities

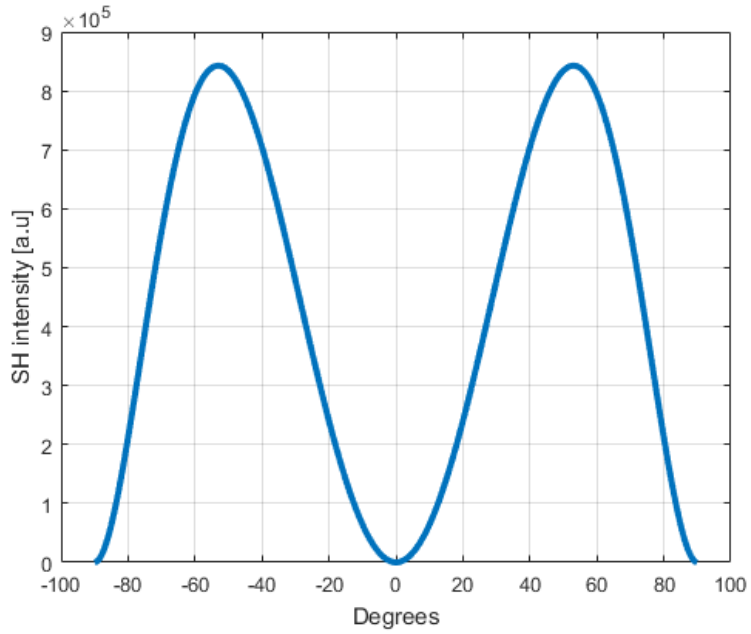

(b) Thin nonlinearity

Figure 1.7: Maker fringe patterns of different types of nonlinearity profile

profiles of sodium, potassium, and silver at the core-cladding interfaces.

Luo et al. studied a similar waveguide structure, but consisting of a lead glass $(\mathrm{Pb0})$ core [36]. Deriving from the equation that describes the space charge model of charge migration, $\chi_{e f f}^{(2)} \sim 3 \chi^{(3)} E_{d c}$ (discussed in section 1.3), it is plausible that a material with higher $\chi^{(3)}$ would posses higher $\chi^{(2)}$ upon poling. So $\mathrm{PbO}$ was chosen because its third-order susceptibility, $\chi^{(3)}$ is in the range of $2-9 \times 10^{-21}$. This is an order of magnitude higher than that of fused silica $\left(2 \times 10^{-22}\right)$. Following thermal poling, scanning a probe laser beam across the sample revealed SHG peaks at the two interfaces on either side of the $\mathrm{PbO}$ core. The $\mathrm{SON}$ induced in the $0.2 \mu \mathrm{m}$ wide $\mathrm{PbO}$ 


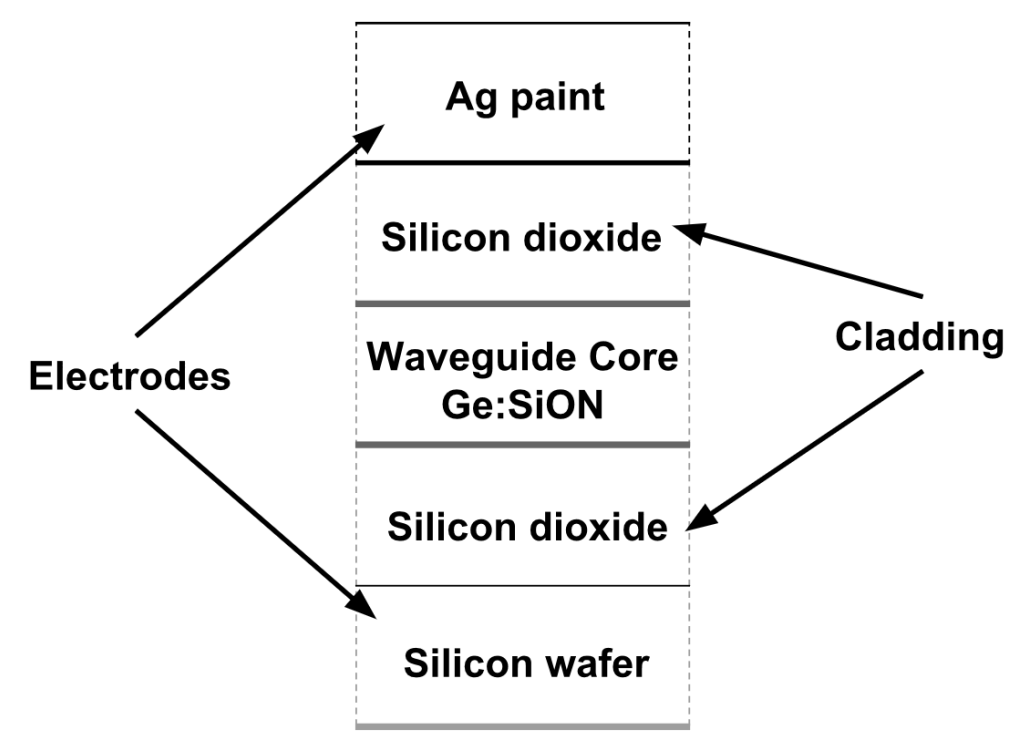

Figure 1.8: Waveguide layers fabricated by Arentoft et al.

layer was calculated to be $\sim 15 \mathrm{pm} / \mathrm{V}$, with an opposite polarity to that of silica.

In 2005, Fleming et al. reported a hindering effect of the migration of the nonlinearity layer at the core-cladding interface [37]. The structure was a D-fiber with a germanium doped core. SH microscopy experiments revealed that the interface impeded the nonlinearity layer at thermal poling voltages lower than $2.5 \mathrm{kV}$. The layer pushed through the interface at higher voltages. In another study [38], they researched on the effect of poling time on the location of the nonlinearity layer in the D-fiber. The nonlinearity layer was shown to travel deeper through the cladding and towards the core with the increasing poling time. In a poling period of 45 minutes, it reached the core-cladding interface, at which point the blocking effect occurred.

Faccio et al. also reported the charge blocking behavior of the core-cladding interface in optical waveguides [39].

Fleming et al. developed a multilayer thin film structure, where three Ge-doped silica layers were deposited on top of a $\mathrm{SiO}_{2}$ substrate, as shown in Figure 1.9 [40]. The Ge-doped layers varied in concentration and width. Upon thermal poling, SH microscopy experiments revealed that the induced SON is proportional the Ge concentration in each layer. It also showed peaks in charge concentration profiles at each interface, including the Ge layer and substrate. The interfaces are thought to 


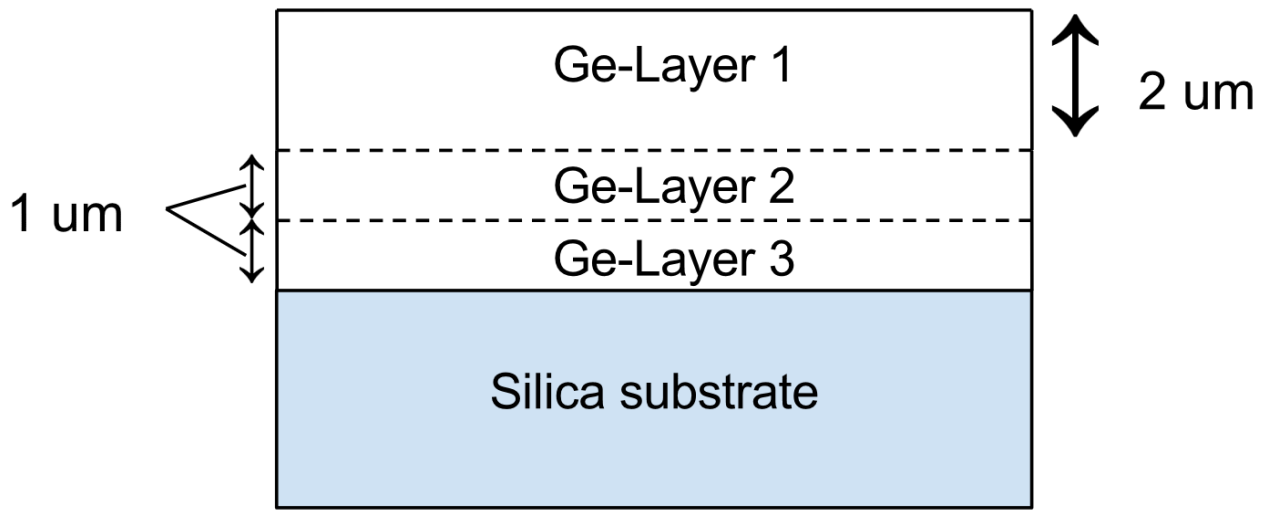

Figure 1.9: Thin film multilayer silica structure developed by Fleming et al.

be hosting layers of opposite charges on either side, thus forming a localized electric field. Due to the narrow width of alternating charge accumulated regions, the net electric field becomes large.

Yadav et al. extended the idea of interfaces to an alternating multilayer silica structure, as shown in Figure 1.10 [41]. In this scheme, they aimed to increase the extent of the nonlinear region by controlling some parameters of the ion charge migration in the sample, such as depth and extent. An extended nonlinear region will allow sufficient overlap between the nonlinearity and optical field propagating through the region, resulting in efficient nonlinear interaction. The multilayer thin film samples were poled using the corona poling method. A femtosecond laser was used for characterization using the Maker-Fringe method. The first set of experiments tested the effect of boron and phosphorous as dopants in alternate layers. Both dopants produced SH intensity higher than that of poled silica. However, the peak for phosphorus was higher by one order of magnitude, whereas for boron it was less than two times. The higher SH intensity is said to occur due to the manner phosphorous bonds within the silicon dioxide lattice. In the following set of experiments, the samples were poled at different voltages, and their characterization showed that the SH intensity increased with increasing poling voltage. To study the distribution of the induced nonlinearity, the final set of experiments involved etching of the thin film samples in dilute hydrofluoric acid. Etching on the anodic side showed the nonlinearity region to extend over a depth of 6 to $10 \mu \mathrm{m}$ in the sample. Interestingly, etching on the cathodic side showed the same result, which is contrary to that observed for poled bulk silica. In poled bulk silica, the induced nonlinearity on the cathodic side is either a thin layer or nothing at all. Thus, equal nonlinearity in both stacks proved that 
the two regions are getting poled simultaneously.

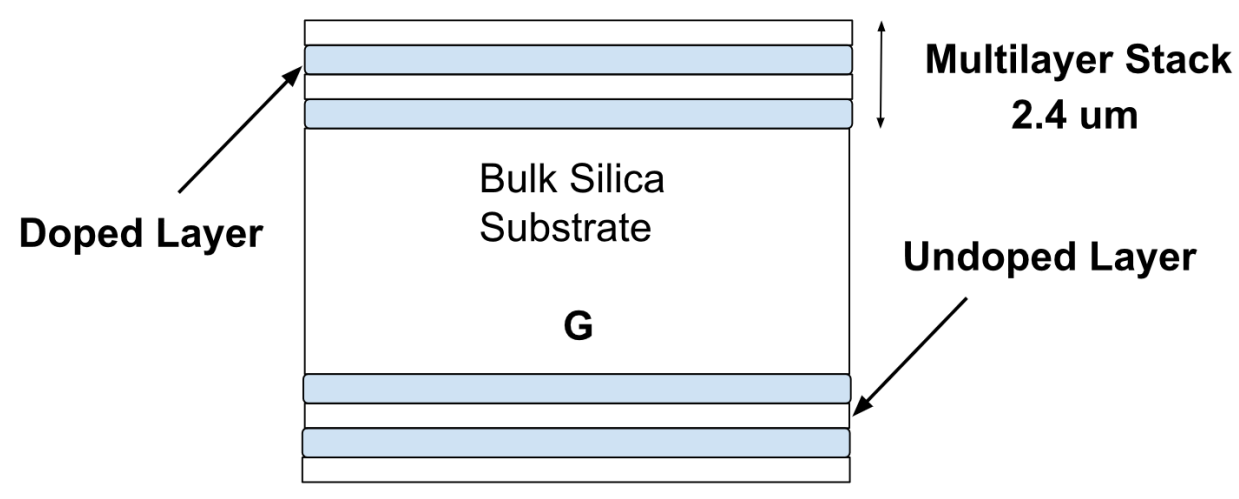

Figure 1.10: Thin film multilayer silica structure with alternating doped layers, developed by Yadav et al.

In another study by Yadav et al., a thermally poled multilayer silica structure produced 204-fold greater SH intensity than that of poled silica [42]. The structure is shown in Figure 1.11. It consists of 40 alternating Ge-doped layers on one side only, each of which was $75 \mathrm{~nm}$ in width. Several possible factors are said to be contributing to this improvement. One reason is that each interface is blocking the migration, hence causing accumulation of alkali impurity ions, resulting in a strong localized electric field. The interfaces may also be blocking the injected positive ions from the anode, thus preventing the neutralization of the negative space charge depletion region. Another possibility is that the lower ionic conductivity of the Ge-doped layer, compared to the undoped layer, may be hindering the charge migration in and of itself. Furthermore, the higher $\chi^{(3)}$ of the Ge-doped layer relative to the undoped silica layer, contributes to a higher SH intensity or $\chi^{(2)}$ (since $\chi^{(2)}$ is proportional to $\chi^{(3)}$ according to equation (1.1)). Besides, etching experiments showed that most of the nonlinearity existed in the region of the stack of layers, which proved the charge blocking capability of the interfaces and the fact that the multilayer silica structures can indeed gain control over the thickness and location of the induced nonlinearity. 


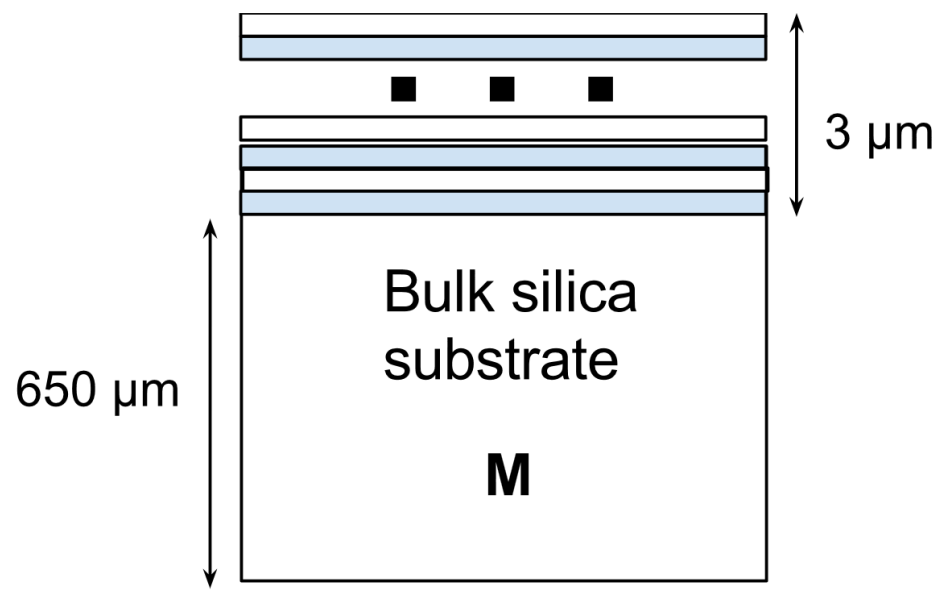

Figure 1.11: Multilayer silica structure with forty alternating doped layers, developed by Yadav et al.

\subsection{Poling of Single Stack Multilayer Structures with Alternate Voltages and Electrodes}

The discovery of the sharp rise in SHG at silica-dopant interfaces and the subsequent development of multilayer silica structure introduced a new technique of enhancing nonlinearity in silica glass. Despite the fundamental physical constraints encountered in silica, the model of alternating multilayer structures developed by Yadav et al. has shown appreciable prospects of improving on the induced SON. Therefore, this research project investigates this model further by studying the effect of poling thin film silica samples with different orientations and voltage polarities. The induced nonlinearity profiles are of particular interest because it can aid in the understanding of the much debated charge migration mechanism of poled silica glass.

To reiterate, the purpose of this design of multilayer structure is to raise the magnitude of the effective nonlinearity by inducing a sufficiently large nonlinear area upon poling. The silica-dopant interfaces will help control the properties of the nonlinear region (e.g. width and distribution). Although each interface has a small nonlinear coefficient (typically $\sim 1 \mathrm{pm} / \mathrm{V}$ ), a larger cumulative thickness of nonlinearity will have a greater overlap with the fundamental wave. This attribute in effect allows for an enhanced effective SON. This approach contrasts with other methods (e.g. various poling techniques), which aim to only increase the nonlinear coefficient of the induced $\mathrm{SON}$ in silica glass. 
In this project, a picosecond laser source pumps the second harmonic in the multilayer structures. This characterization procedure is unlike that of Yadav et al. which

used a femtosecond source. For this study, the advantage of a picosecond laser is that the beam pulses have longer lengths which allows better overlap between the SH waves being generated by the fundamental wave, over one coherence length [43]. Thus, pulse propagation effects due to the different group velocities of the fundamental and the SH waves are insignificant compared to the physical overlap of the waves. This phenomenon would produce characteristic modulation fringes in the Maker fringe envelope, which may be unique to the novel design of the multilayer structures.

\subsection{Thesis Organization}

Figure 1.12 shows a progression diagram of this research project. The numbers 1 to 4 label the different stages. Stages 1, 2 and 3 are the focus of chapter 2. It describes the design of the samples and the poling and characterization procedures. It also includes a brief analysis of experimental errors due to apparatus used in this project, followed by a description of the steps undertaken to ensure maximum accuracy in the experimental results. Stage 4 is described in Chapter 3. It presents the results obtained from the Maker fringe experiments followed by an in-depth analysis of induced nonlinearity of the multilayer structures. The experimental observations are explained using a hypothesis in Chapter 4, that is based on a MATLAB simulation model. The final chapter provides some concluding remarks on the research findings and indicates possible aspects and methodology for future work. 


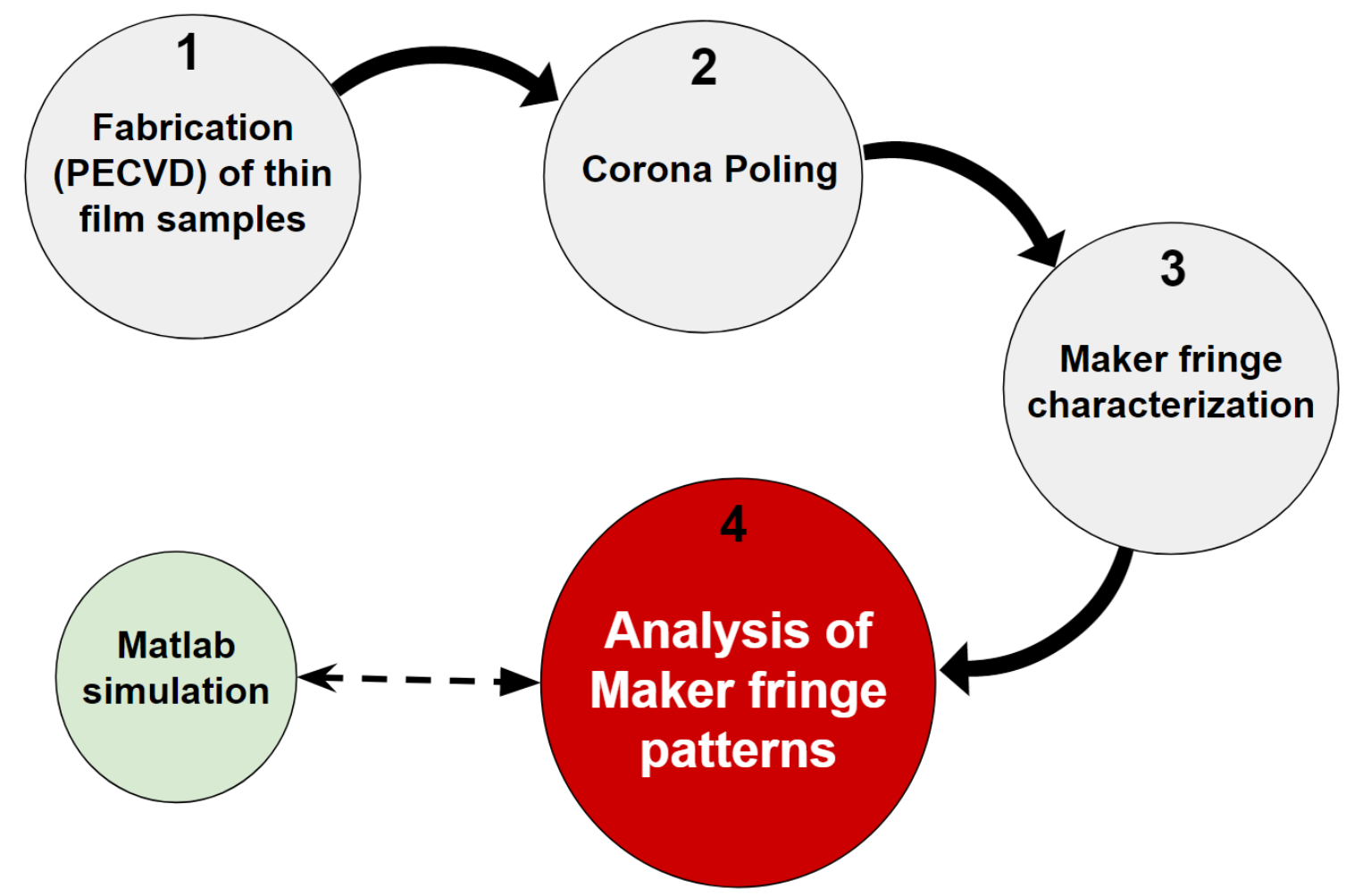

Figure 1.12: Progression diagram showing the various stages in this research project 


\section{Chapter 2}

\section{Experimental Procedure}

In this project, the multilayer thin film samples were developed using plasmaenhanced chemical vapor deposition (PECVD), facilitated by CMC Microsystems. During the process, $500 \mu \mathrm{m}$ thick fused silica substrates, manufactured by University Wafer, are deposited with eight alternating doped and undoped silica layers. Fused silica is an amorphous form of glass with very lower impurity content. Its high level of purity makes it preferable for semiconductor fabrication and research studies [44]. The doped layers consist of germanium, with a concentration of $5 \%$ across all layers for each sample. The concentration level of $5 \%$ was found to be suitable in another ongoing study in this research group, the details of which is outside the scope of this thesis. Figure 2.1 shows the multilayer structure and the relevant dimensions.

\subsection{Poling Setup}

Corona poling is the primary method used to induce nonlinearity in the samples. The electric field is applied with a needle-to-plane electrode geometry, like shown in Figure 1.1b. The poling conditions are identical to the ones described in section 1.1.1, except that the high voltage tungsten needle is $2.8 \mathrm{~cm}$ above the sample, instead of the typical $\sim 1 \mathrm{~cm}$. The increased height is to ensure a uniform and perpendicular electric field distribution over the sample surface. The voltage at the tungsten needle is 10 to $11 \mathrm{kV}$. The net electric field is calculated to be 355 to $390 \mathrm{~V} \mathrm{~mm}^{-1}$. This electric field is appropriate to ensure that no arcing occurs in the air between the two electrodes, and support charge migration to induce $\mathrm{SH}$ in the samples. 


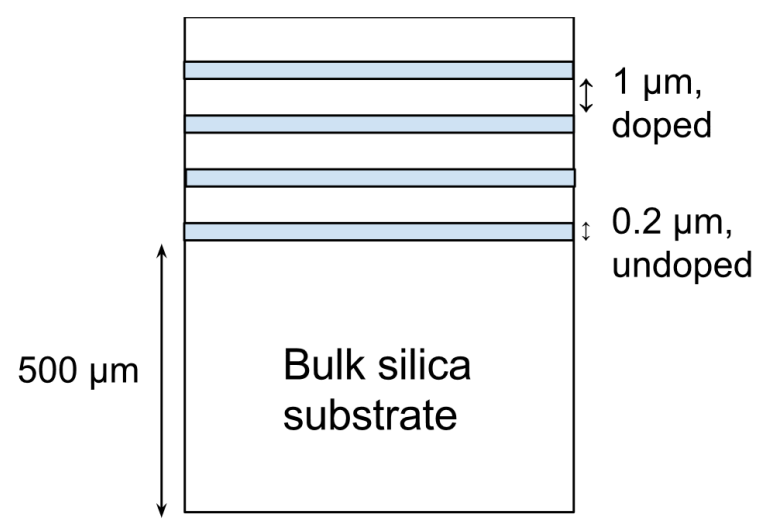

Figure 2.1: Thin film samples with 8 alternating layers

Figure 2.2 shows six different poling configurations, including poling of bulk silica (cases 2.2a and 2.2b). Bulk silica is poled to be able to compare with poled multilayer structures. Cases 2.2c, 2.2d, 2.2e, and 2.2f show four poling configurations for physically identical samples. The sample orientation and poling voltages change across the configurations. For ease of identification, the samples are labeled as SPbulk, SNbulk, SPup, SPdown, SNup, and SNdown. Here the letters 'P' and 'N' stand for positive and negative poling, respectively. The word 'up' means multilayer stack facing the high voltage tungsten needle, whereas the word 'down' means the multilayer stack facing away from the needle. A detailed specification of the poling configurations is listed below.

1. Sample SPbulk is bulk silica. It is poled with a positive voltage at the tungsten needle (anode).

2. Sample SNbulk is bulk silica. It is poled with a negative voltage at the tungsten needle (cathode).

3. Sample SPup is a multilayer structure. It is poled with a positive voltage at the tungsten needle (anode). The side with the multilayer stack faced the anode while the thin-film substrate was in contact with the cathode (ground).

4. Sample SPdown is a multilayer structure. It is poled with a positive voltage at the tungsten needle (anode). However, the side with the multilayer stack is in contact with the cathode (ground), while the thin-film substrate faced upwards towards the anode. 
5. Sample SNup is a multilayer structure. It is poled with a negative voltage at the tungsten needle (cathode). The side with the multilayer stack faced the cathode while the thin-film substrate was in contact with the anode (ground).

6. Sample SNdown is a multilayer structure. It is poled with a negative voltage at the tungsten needle (cathode). However, the side with the multilayer stack was in contact with the anode (ground), while the thin-film substrate faced upwards towards the cathode.

\subsection{Maker Fringe Setup}

The Maker fringe measurement technique is used to characterize the generated $\mathrm{SH}$ power in the poled samples. Figure 2.3 shows the Maker fringe setup. The source is a diode pumped solid state picosecond laser, operating at a wavelength of $1064 \mathrm{~nm}$, repetition rate $500 \mathrm{~Hz}$, average power $152 \mathrm{~mW}$, and $10 \mathrm{ps}$ pulse width. The aperture diameter (beam size) is $\sim 3 \mathrm{~mm}$. At the output of the source, two lens of $5 \mathrm{~cm}$ and $2.5 \mathrm{~cm}$ collimates the beam. A low-pass filter in between the two lens eliminates parasitic SHG in the beam path. A Glan-Thompson polarizer allows passing of light with a specific polarization, which in this case is horizontal polarization. A halfwave plate rotates the polarization of the linearly polarized laser light (incoming beam). In this case, it is adjusted so that the average power of the incident beam at the sample is $\sim 2.1 \mathrm{~mW}$. A $15 \mathrm{~cm}$ lens focuses the beam at the sample with an intensity of $1.49 \times 10^{10} \mathrm{~W} / \mathrm{cm}^{2}$. The sample is on a motorized rotation stage. A $10 \mathrm{~cm}$ lens collimates the beam again after passing through the sample. A highpass filter eliminates radiation above $800 \mathrm{~nm}$. A prism separates out the $\mathrm{SH}$ signal of $532 \mathrm{~nm}$ from the remaining $1064 \mathrm{~nm}$ fundamental wave. A bandpass filter at the input of a photomultiplier tube (PMT) eliminates stray light and only allows SHG of wavelength $532 \mathrm{~nm}$ to pass through. A Labview program controls the motorized stage holding the sample. The stage rotates while the PMT detects the SH peak power from the sample at each incidence angle. A plot of incidence angle versus SH power is generated to characterize the sample. 
SPbulk

$+$

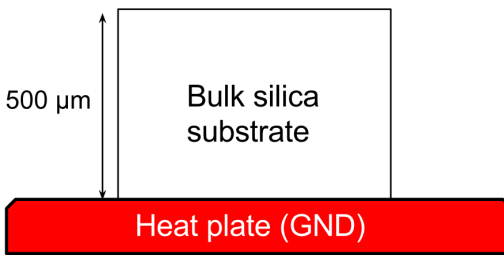

(a) Positive voltage; silica substrate.
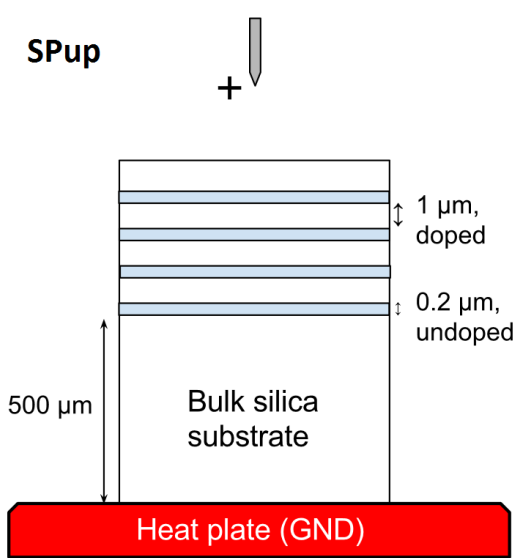

(c) Positive Voltage; layers facing up.
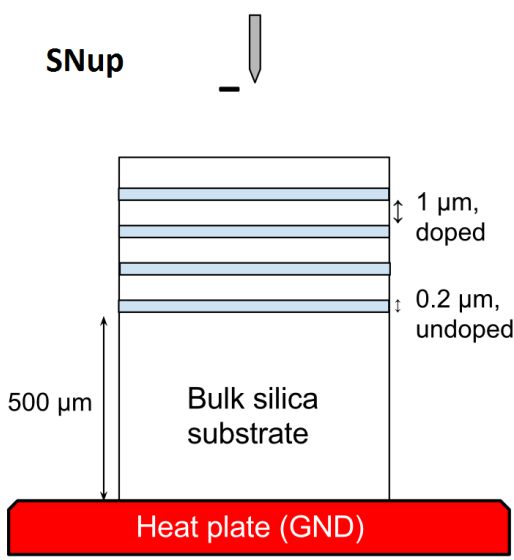

(e) Negative Voltage; layers facing up.
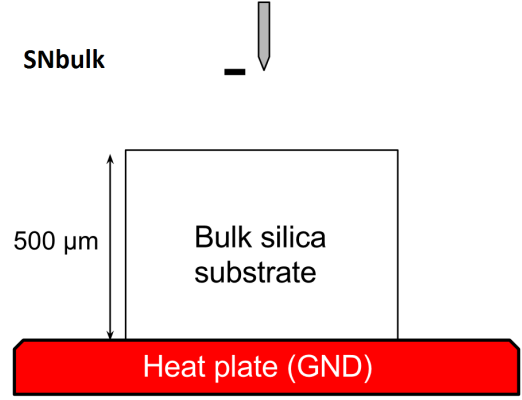

(b) Negative Voltage; silica substrate.

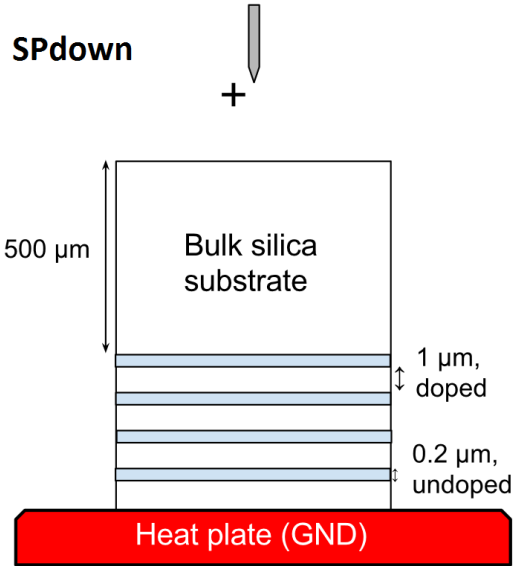

(d) Positive Voltage; layers facing down.

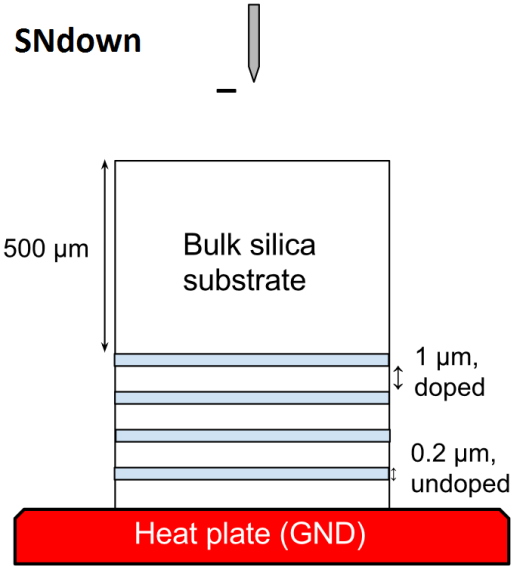

(f) Negative Voltage; layers facing down.

Figure 2.2: Setup diagrams for poling voltage and orientation. 


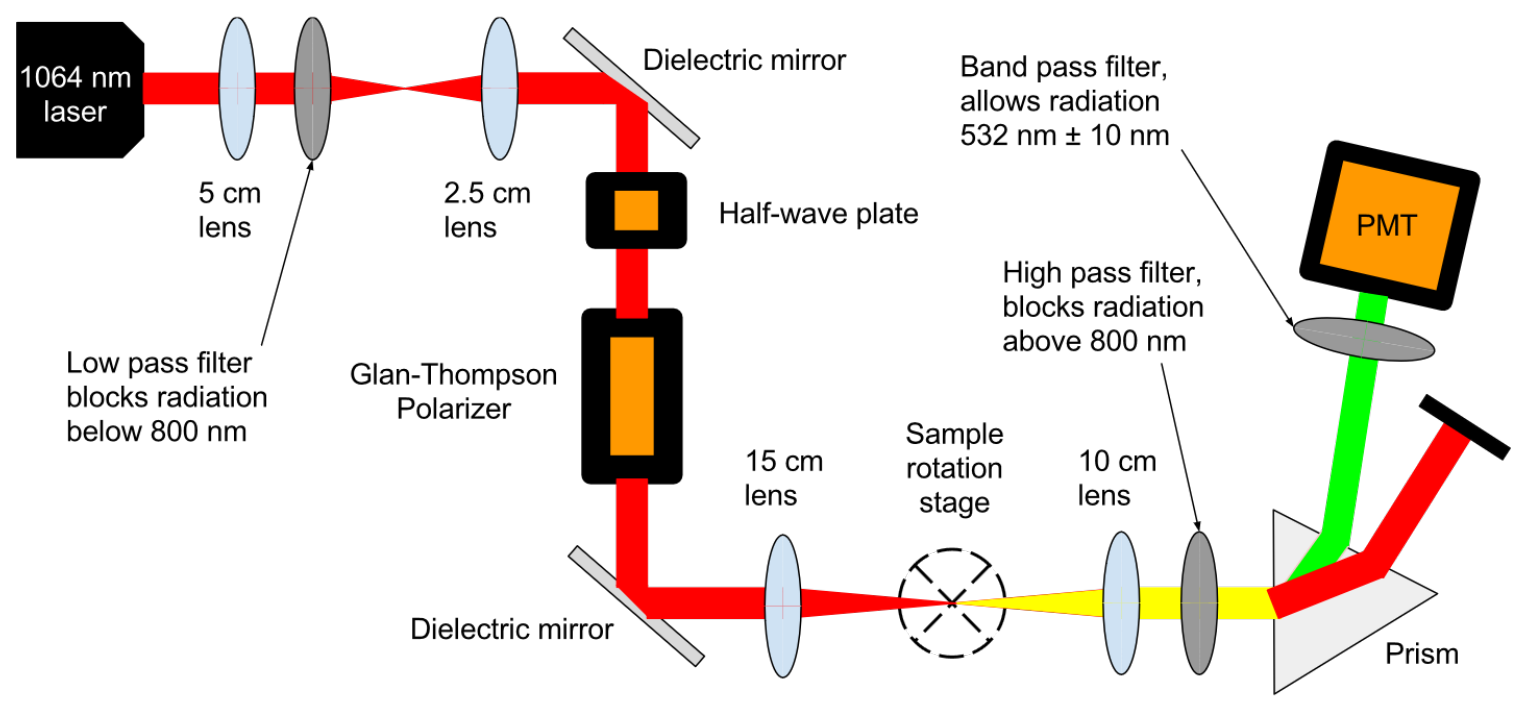

Figure 2.3: Setup for Maker Fringe Experiments

\subsection{Measurement Apparatus, Experimental Con- ditions, and Errors}

This section provides an overview of the conditions and the associated errors in the experimental procedure described in this chapter. It is important to locate, understand and minimize the effect of inconsistencies throughout the experimental lifecycle, to attain maximum accuracy and ensure correct analysis of the data.

\subsubsection{Corona Poling}

The corona poling setup described in 2.1 is used to pole the entire set of samples shown in Figure 2.2. Ideally, all the samples would be poled and characterized in the same set of an experimental run, to ensure consistency in all conditions such as poling voltage, temperature, laser beam power, the polarization of light, and humidity and temperature of the atmosphere. In this project, apart from samples SPbulk and SNbulk, all the rest were poled and characterized in the same set.

The necessity of using poled bulk silica substrate as a reference was realized only after observing distinct characteristics in the Maker fringe patterns from the poled multilayer structures. Therefore, comparisons between SH peaks of Maker fringe 
patterns derived from poled bulk substrates with that of poled multilayer structures should be only general rather than exact (as will be demonstrated in Chapter 3).

High voltage DC converters from EMCO, models DX250 and DX250N (for positive and negative, respectively) supply the voltage at the tungsten needle. The swapping between modules to alter the voltage polarity may introduce discrepancies in the experimental conditions. A Fluke $80 \mathrm{~K}-40$ high voltage probe measures the absolute values of the voltages which are kept within an accuracy of $3 \%$ of $10.5 \mathrm{kV}$.

A K-type scientific digital thermometer DM6801 is used to measure the poling temperature.

It is not a requirement in this project to optimize or customize poling methods

for the multilayer structures. Rather, standard poling parameters are maintained to allow direct comparison with related studies in the literature. Optimization of poling conditions for these new structures can be a subject of study in and of itself.

\subsubsection{Maker Fringe Experiment}

The Maker fringe setup described in 2.2 is used to characterize the entire set of samples. Referring to Figure 2.3, a Thorlabs S314C power meter measures the output power of the laser beam at a location between the $15 \mathrm{~cm}$ focusing lens, and the sample. At the beginning of every experiment, a set procedure is carried out (as described in the next paragraph) to ensure stable and consistent experimental conditions.

The half-wave plate allows adjustments for the desired power of the laser beam. The focal point is checked for its location to be at the center of the thin film sample. Lithium niobate, an efficient nonlinear crystal is used to visibly check for the path of the SHG beam through all the optical components, including the aperture of the PMT (Hamamatsu H7827-001 19 mm diameter voltage-type photomultiplier tube). Stray light and reflectance, which are dependent on sample thickness, are minimized by covering the edges of the PMT aperture with black non-reflective surfaces. The $\mathrm{SH}$ signal from the samples is recorded in real time, without any delay and averaging. The first Maker fringe experiment is run on an x-cut quartz thin film. Figure 2.4 shows a sample Maker fringe pattern obtained from a quartz crystal during one of the experimental runs. The number of peaks and troughs and SH intensity of the Maker fringe envelope is compared to a reference. Thus, the setup is aligned on an as-needed basis. 


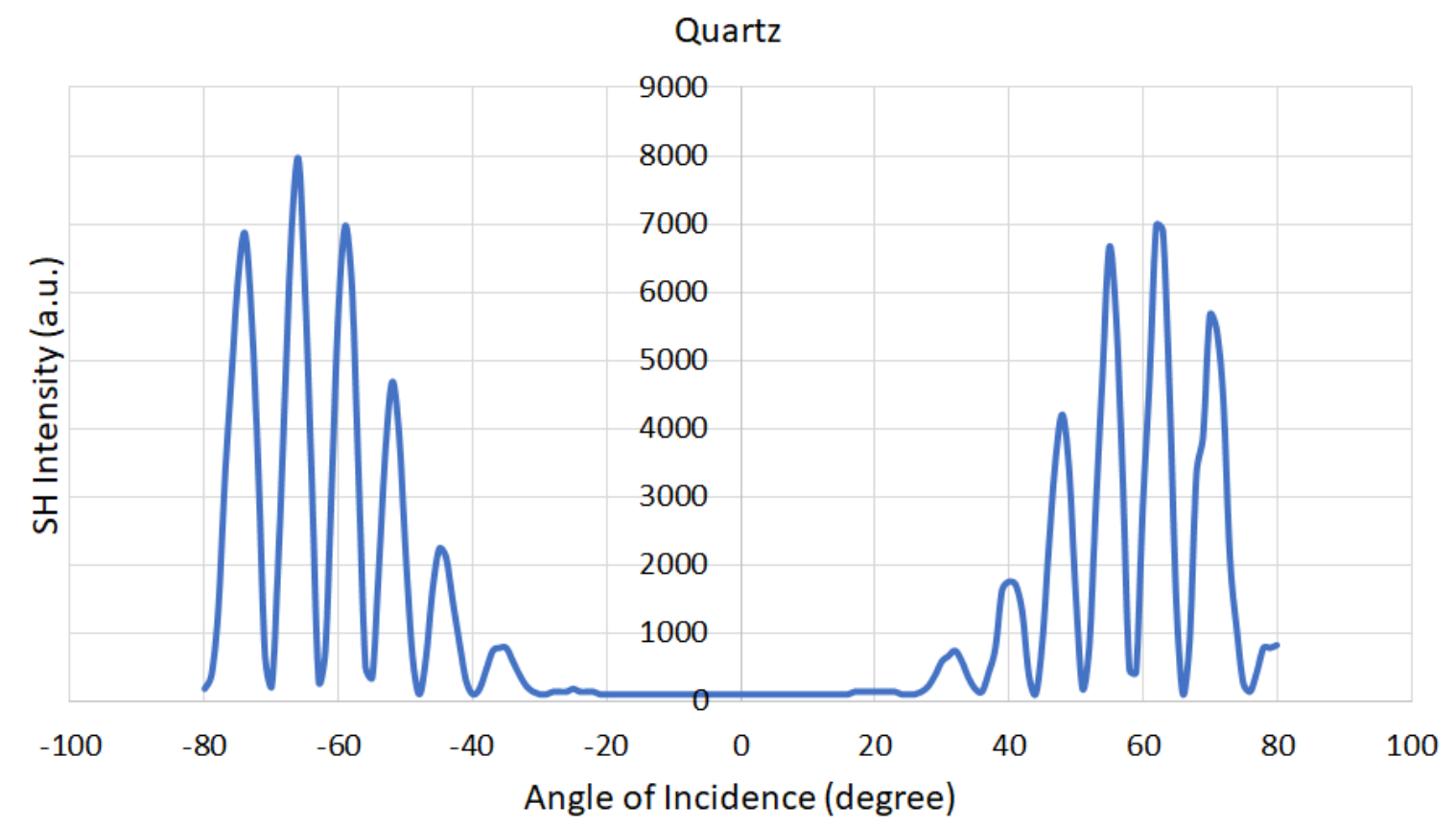

Figure 2.4: Maker fringe pattern obtained from a Quartz sample

It is also important to estimate the errors of the two variables used to plot the Maker fringe graphs, which are the input pump power and the SH intensity. Through repeated measurements from a single experiment, the extent of error has been estimated to be $\pm 2400 \mathrm{mV}$ and $\pm 20.25 \mathrm{~mW}$ for the PMT and the power meter, respectively. 


\section{Chapter 3}

\section{Maker Fringe Characterization}

This chapter presents the results obtained from the experiments described in chapter 2. Figure 2.2 showed the different sample orientations during the poling stage. The results from the Maker fringe experiments are characterized using the charge migration model discussed in 1.3. However, one should note that the real charge migration model for samples in this research project may be different. This project utilizes the corona poling method for inducing nonlinearity. Existing information in the literature is predominantly based on thermal poling. Due to the unavailability of sufficient data of corona poled silica glass, the charge migration mechanism in the current samples is assumed to be the same as for thermal poling.

This chapter also presents a hypothesis for the induced nonlinearity, which will be verified using a MATLAB simulation model in chapter 4 .

\subsection{Maker Fringe Patterns}

\subsubsection{Positive poling}

\section{Bulk Silica Substrate, SPbulk}

Figure 3.1 shows the Maker fringe pattern obtained from sample SPbulk. It shows two lobes on either side of the normal with no modulation fringes. This suggests that the width of the induced nonlinearity is less than the coherence length, $L_{c}$. Assuming that the nonlinearity is uniform, using equation $1.2, L_{c}$ is calculated to be $23.96 \mu \mathrm{m}$, where $\omega=1064 \mathrm{~nm}, 2 \omega=532 \mathrm{~nm}, n_{\omega}=1.4496$, and $n_{2 \omega}=1.4607$. As discussed in 1.3, the nonlinear region of width 2 to $14 \mu \mathrm{m}$ is expected to form beneath the anode 
which is less than the $L_{c}$ in this case.

Figure 3.7a shows a model of nonlinearity layer formation in sample SPbulk.

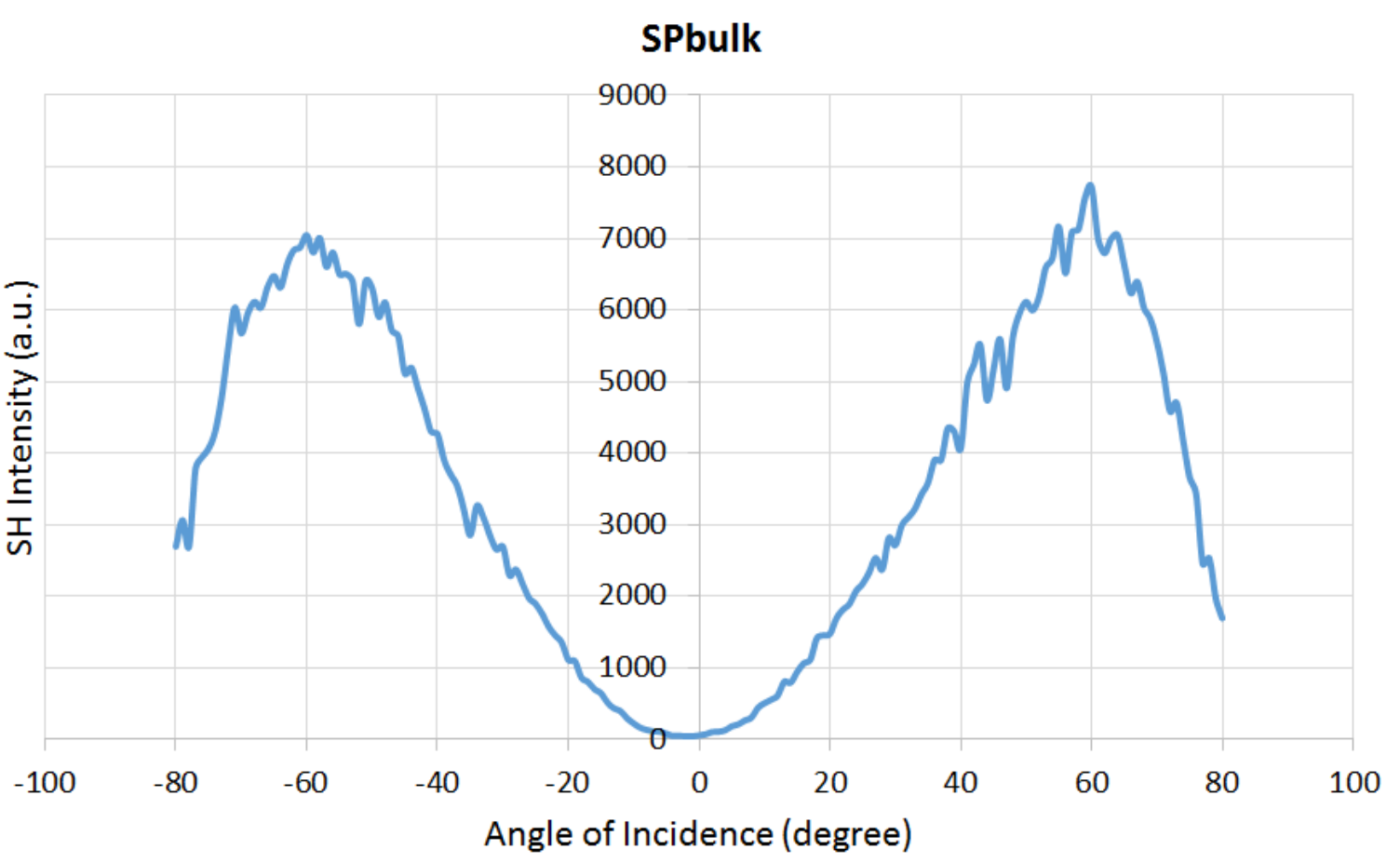

Figure 3.1: Maker fringe pattern for SPbulk.

\section{Multilayer Silica Structure with Layers Facing Anode, SPup}

Figure 3.2 shows the Maker fringe pattern obtained from sample SPup. Unlike the case of sample SPbulk, deep modulation fringes are apparent in the pattern, depicting a dissimilarity between nonlinearities induced in the two structures. The most likely cause of modulation is the formation of two nonlinear layers in the sample during poling. The SHG from the two layers interacts to cause destructive and constructive interference. Since the addition of the $4.8 \mathrm{\mu m}$ thick multilayer stack brought about the modulation, it is reasonable to assume that one of the nonlinear layers is in the stack itself. Then the other nonlinear layer would be located at an appropriate depth inside the silica substrate.

One should also note that the deep modulation in the Maker fringe pattern indicates that the two nonlinear regions are comparable in width. Therefore, the new nonlinear layer is presumably in the range of 2 to $14 \mu \mathrm{m}$ as well. 
Figure 3.7c shows a model of the nonlinear silica sample, SPup. The assumption, for now, is that one of the nonlinear regions is in the multilayer stack, and another one exists on the other side of the sample.

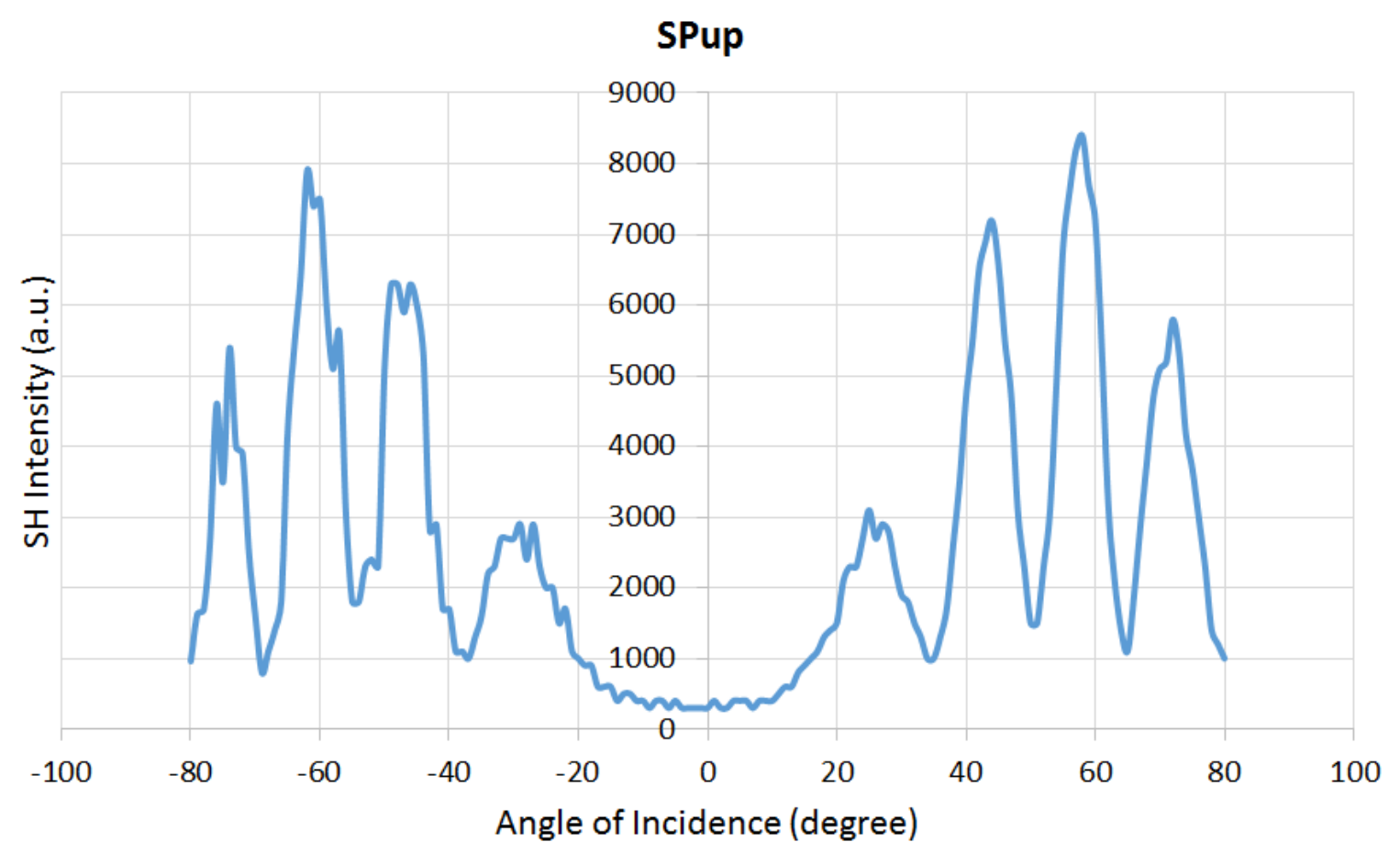

Figure 3.2: Maker fringe pattern for SPup.

\section{Multilayer Silica Structure with Substrate Facing Anode, SPdown}

Figure 3.3 shows the Maker fringe pattern obtained from sample SPdown. No modulation fringes appear in this case, as was the case of sample SPbulk. Therefore, a single layer of nonlinearity is likely forming in the substrate near the anode. Figure $3.7 \mathrm{~d}$ shows a model of nonlinearity.

\subsubsection{Negative poling}

\section{Bulk Silica Substrate, SNbulk}

Figure 3.4 shows the Maker fringe pattern obtained from sample SNbulk. This pattern shows no modulation fringes, like the one from sample SPbulk. Figure 3.7b 


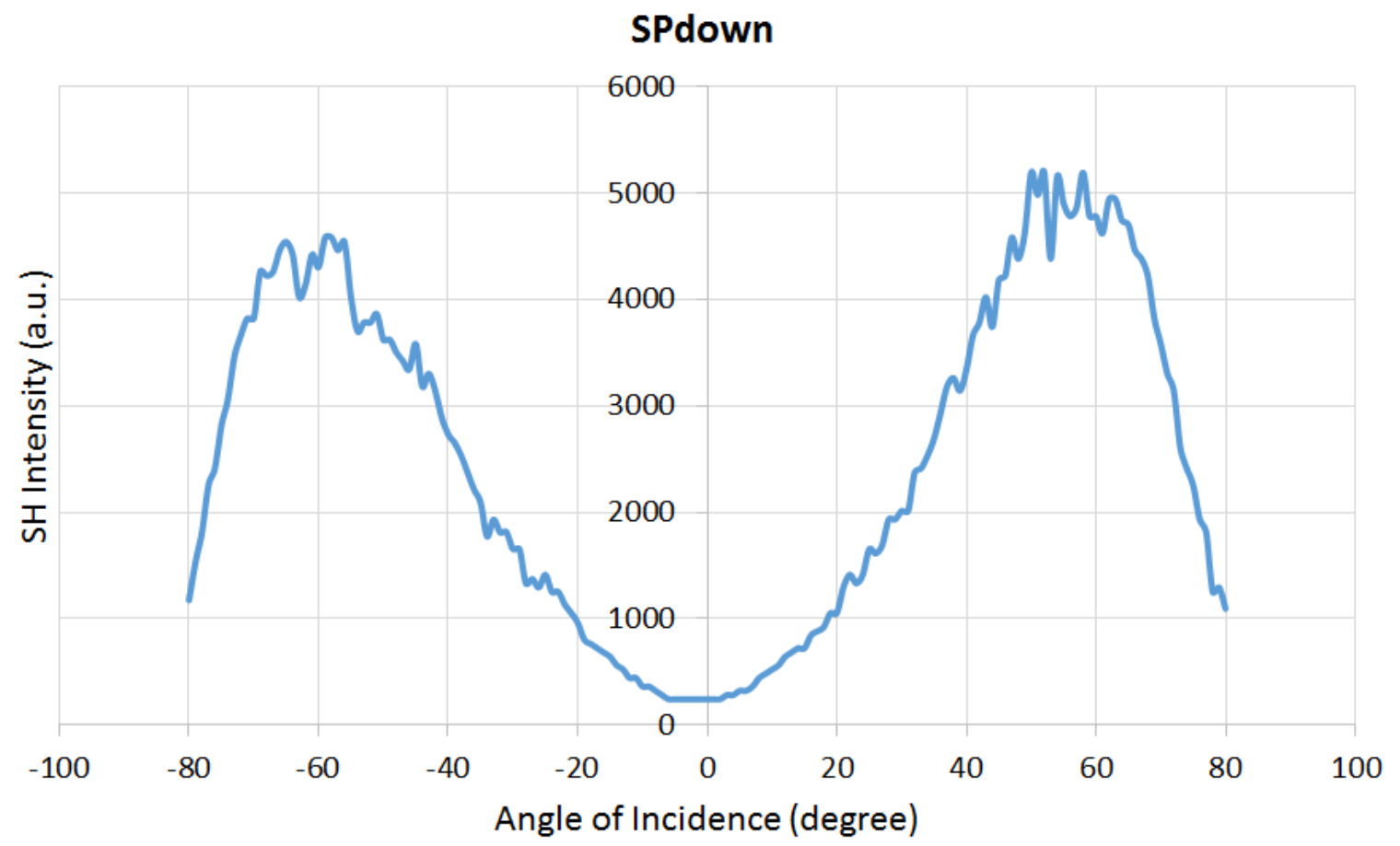

Figure 3.3: Maker fringe pattern for SPdown.

shows a model of nonlinearity in this sample. Applying the charge migration model presented in 1.3, it is most likely that the nonlinear layer will form near the anode, which in this case is the heat plate (ground).

\section{Multilayer Silica Structure with Layers Facing Anode, SNup}

Figure 3.5 shows the Maker fringe pattern obtained from sample SNup. Once again, the pattern of two lobes suggests that only a thin nonlinear layer has formed in the sample during poling. The similarity of this pattern to the one obtained for SNbulk, SPbulk and SPdown (as shown in Figure 3.4, Figure 3.1 and Figure 3.3, respectively) implies that the mechanism of charge migration that cause nonlinearity in these structures is the same all across. Figure 3.7e shows a model of the nonlinear multilayer silica sample. Like all the previous cases, the nonlinear layer is shown to have formed near the anode. 


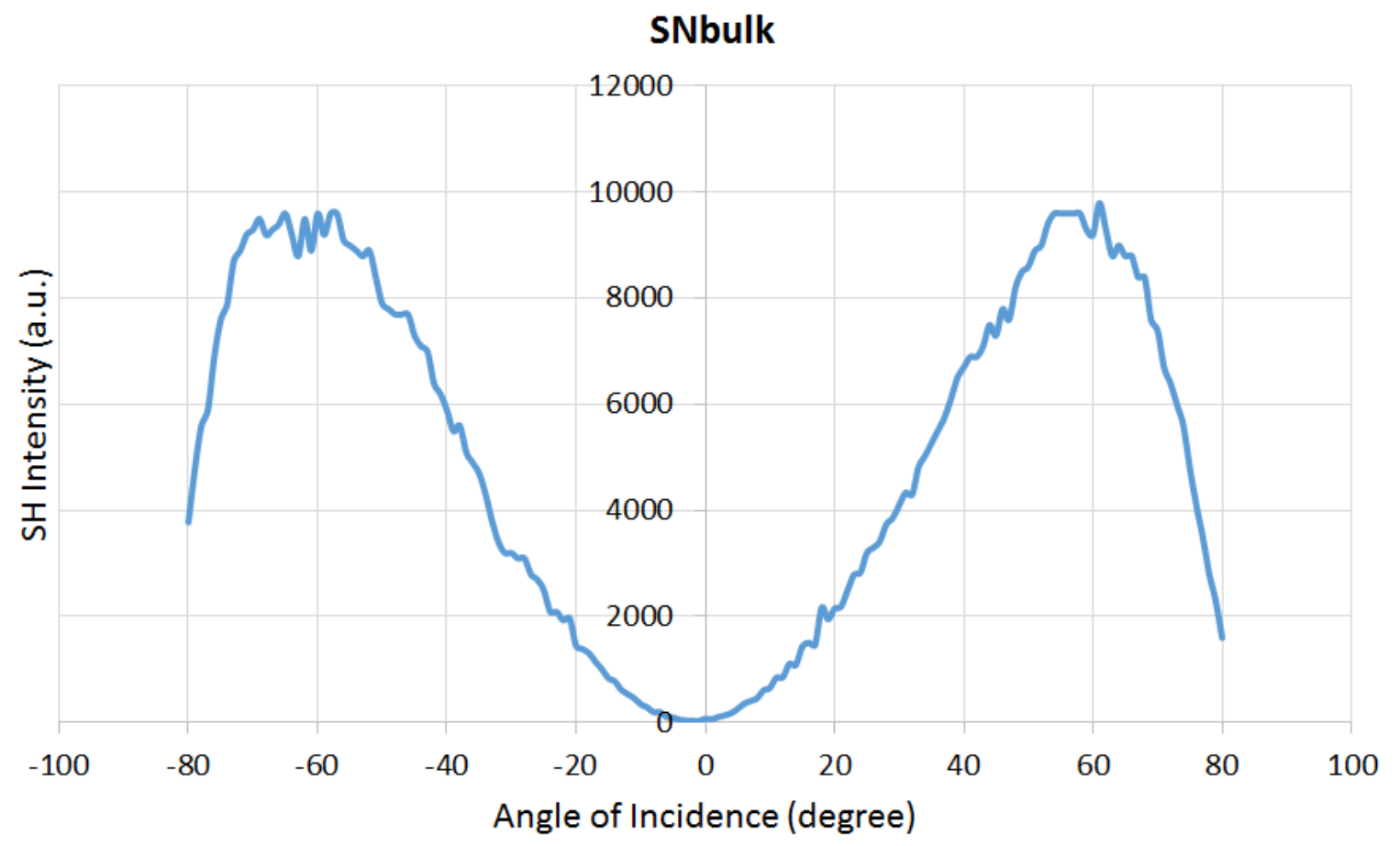

Figure 3.4: Maker fringe pattern for SNbulk.

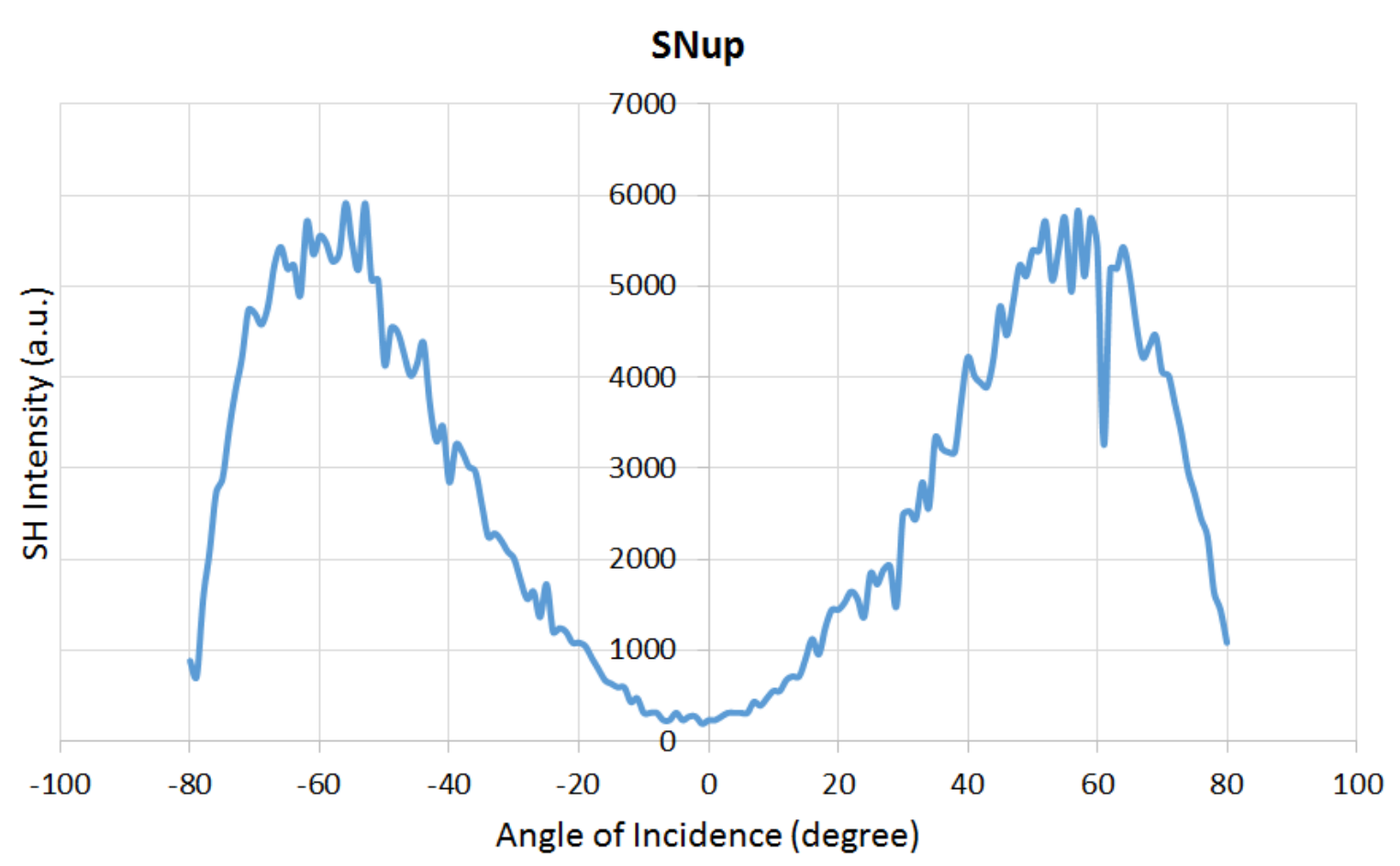

Figure 3.5: Maker fringe pattern for SNup. 


\section{Multilayer Silica Structure with Substrate Facing Anode, SNdown}

Figure 3.6 shows the Maker fringe pattern obtained from sample SNdown. With the reversal of the sample orientation during poling, nonlinearity is induced such that modulation fringes now appear in the Maker fringe pattern. This pattern previously appeared for sample SPup (Figure 3.2), which implies that the charge migration mechanism for samples SPup and SNdown are similar. Therefore, two nonlinear layers are likely being formed in this case as well. Figure 3.7f shows the corresponding model of nonlinearity in the multilayer structure.

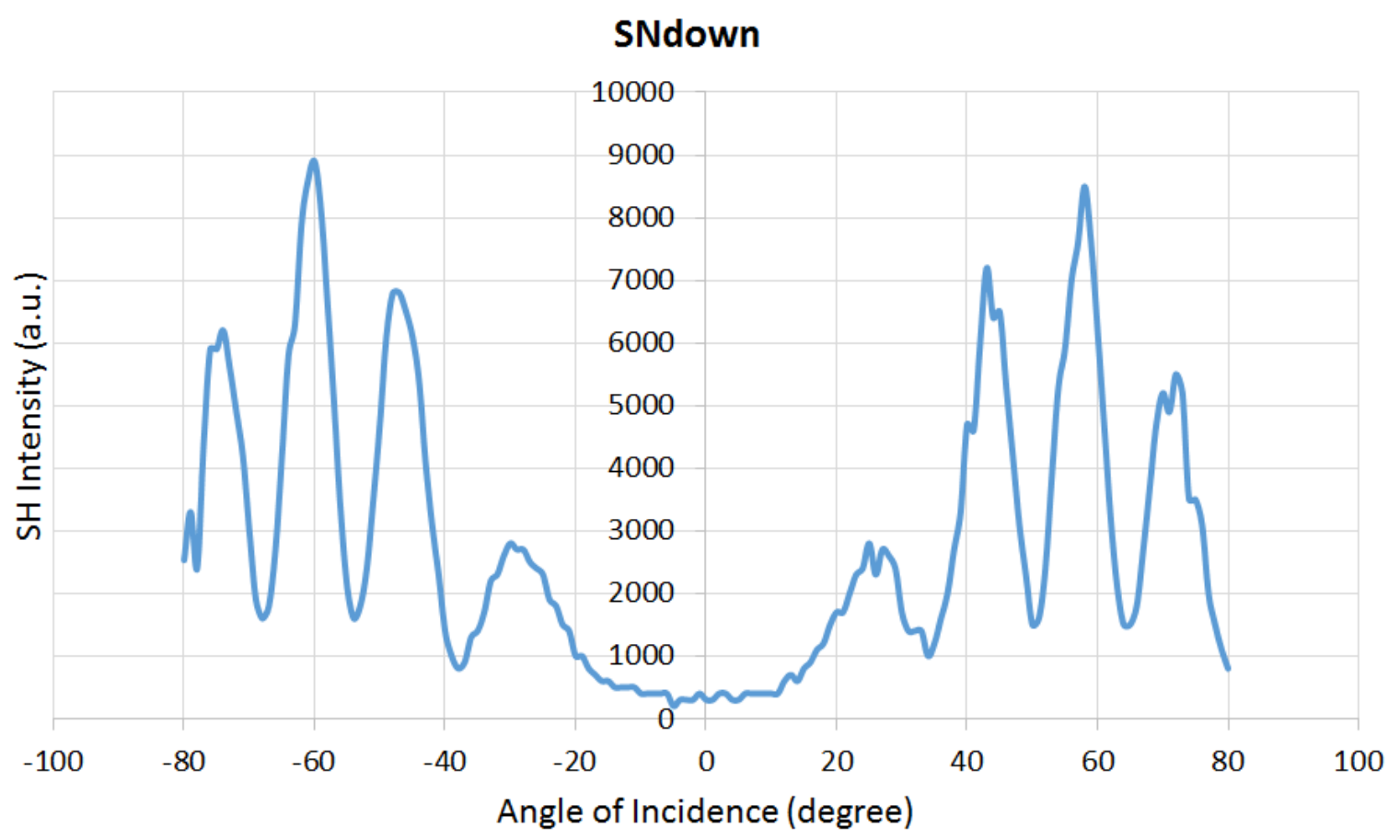

Figure 3.6: Maker fringe pattern for SNdown.

\subsection{Comparison of Maker Fringe Patterns}

This section presents various types of comparisons among the Maker fringe patterns shown in section 3.1, which can help exhibit a few important aspects of the induced nonlinearity of the multilayer structures. 

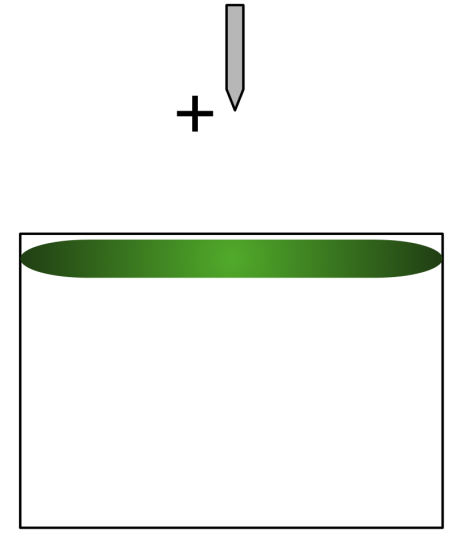

(a) Model for SPbulk
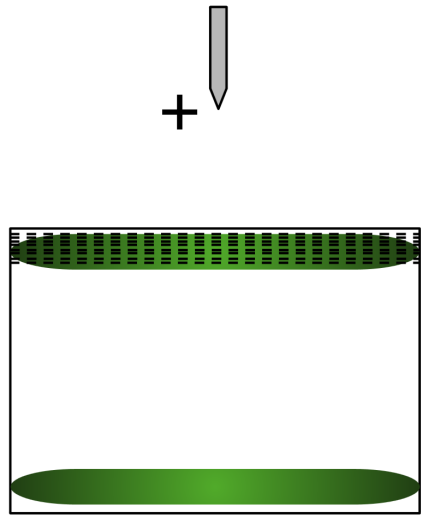

(c) Model for SPup<smiles></smiles>

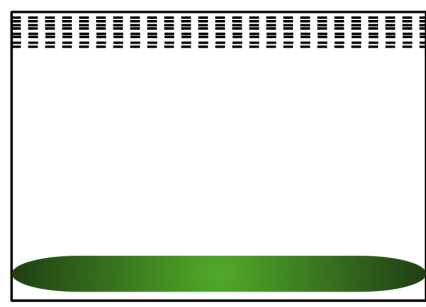

(e) Model for SNup

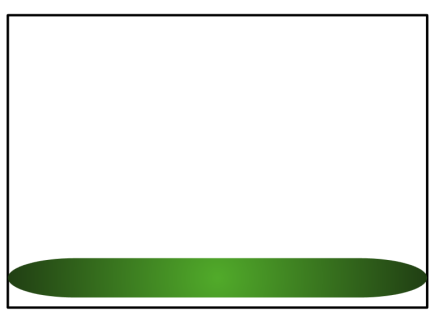

(b) Model for SNbulk
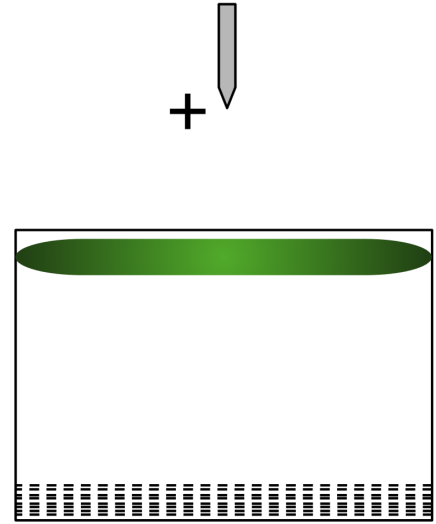

(d) Model for SPdown
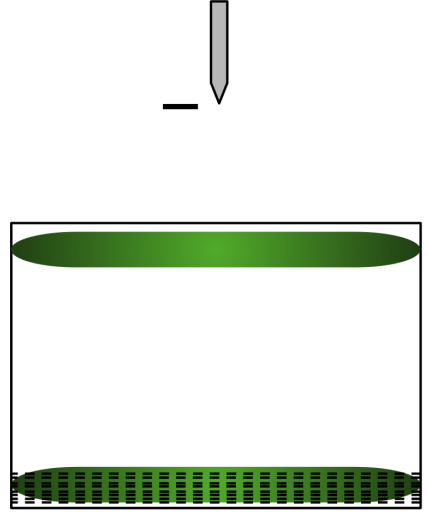

(f) Model for SNdown

Figure 3.7: Charge migration model of the poled samples 


\subsubsection{Similarity Between Maker Fringe Patterns}

Figure 3.8 compares between samples SPup and SNdown. It is apparent that the SH peak, modulation depth and the overall distribution of the two graphs are nearly identical, suggesting that the charge migration mechanism to induce nonlinearity for the samples are same. This reiterates the validity of the presumed similarity between the charge migration models shown in Figure 3.7c and Figure 3.7f.

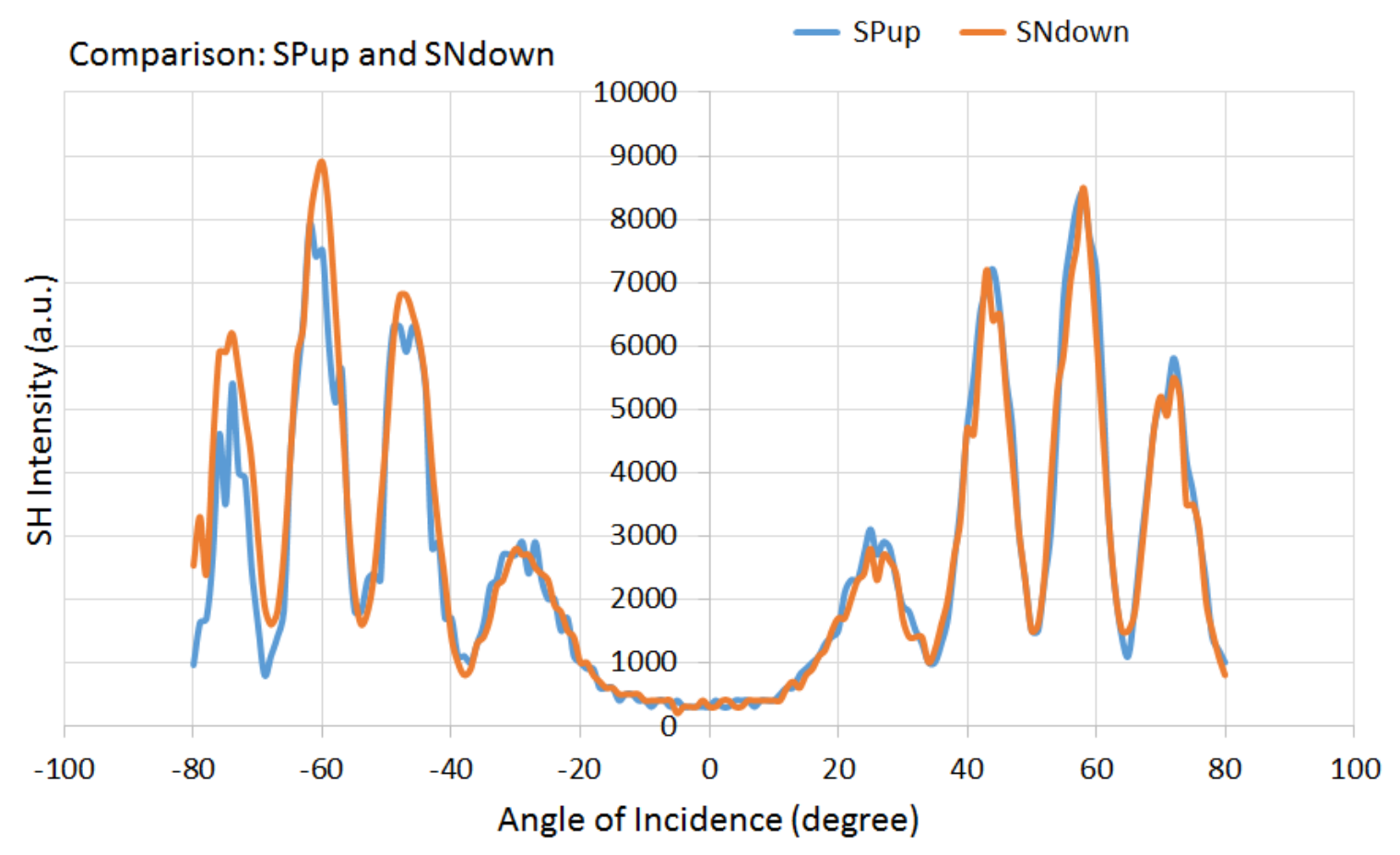

Figure 3.8: Comparison of Maker fringe patterns of SPup and SNdown

On the other hand, Figure 3.9 shows a comparison between samples SPdown and SNup. The Maker fringe patterns from these two samples did not show any modulation fringes. The graphs are very similar apart from a slight variation between the peak intensities at $-60^{\circ}$. This minor difference is an attribute of an experimental anomaly discussed in 2.3. The similarity between these Maker fringe patterns reiterates the validity of the charge migration models shown in Figure 3.7d and Figure 3.7 e. 


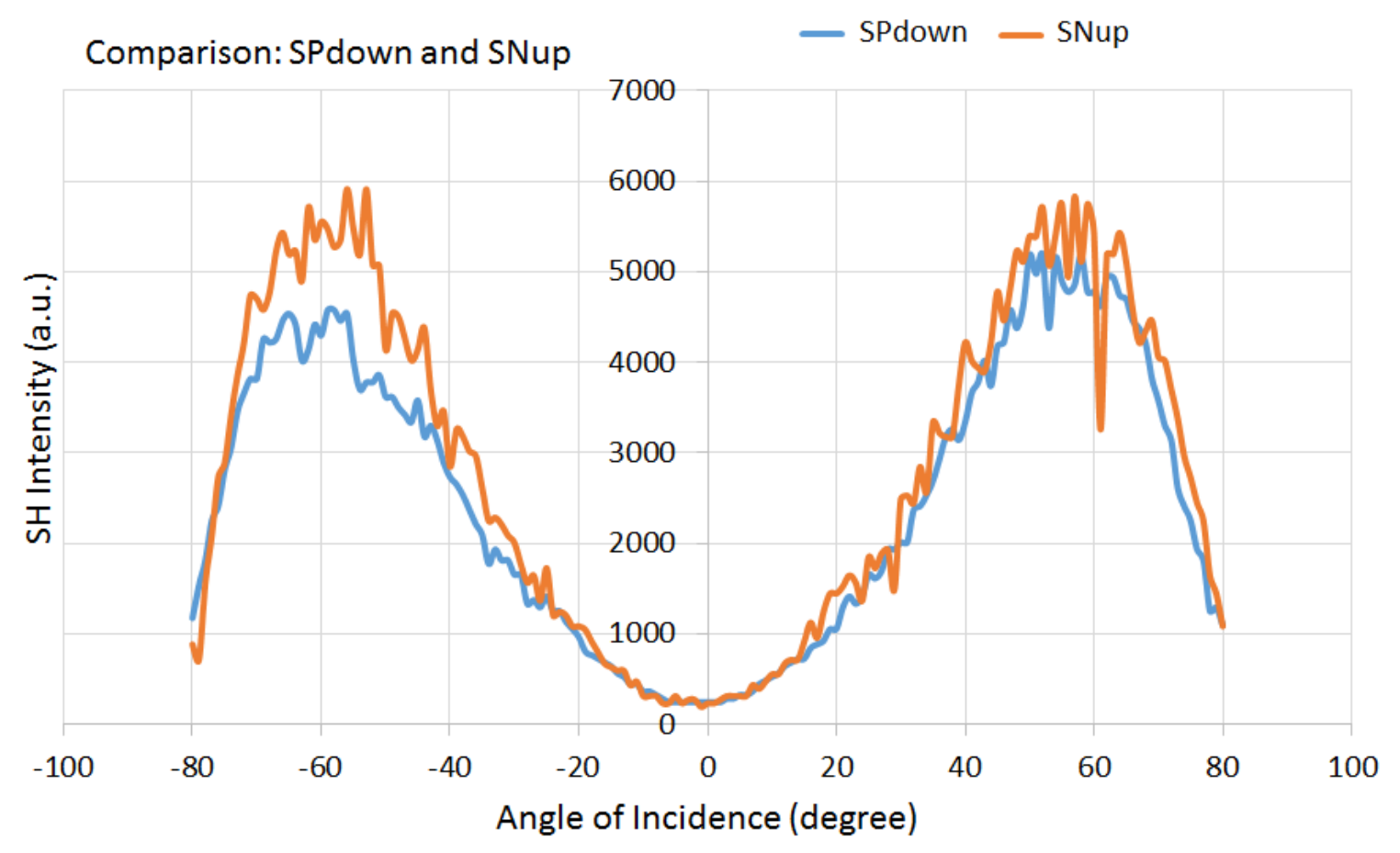

Figure 3.9: Comparison of Maker fringe patterns of SPdown and SNup

\subsubsection{SH Peak Intensities}

This section presents a comparison among peak intensities of like samples, i.e. samples that were poled with the same voltage polarity. The patterns of SPbulk and SNbulk are included for general comparison purposes.

Figure 3.10 shows the Maker fringe patterns of samples SPup, SPdown and SPbulk. The most notable feature of this graph is that the SH peak of sample SPdown, which does not have modulation fringes, is only $\sim 65 \%$ that of SPup. In fact, its peak intensity is the lowest of the three samples.

Figure 3.11 shows the Maker fringe patterns of samples SNup, SNdown and SNbulk. The differences in SH peaks are like those observed for the positively poled samples. The SH peak of sample SNup is the lowest of the three. It is only $\sim 65 \%$ that of the one obtained from sample SNdown, as well.

It is apparent that the lowest $\mathrm{SH}$ peaks occur for multilayer structures that do not have modulation fringes (SPdown and SNup). These samples were poled with the stack of layers facing the cathode. Even though the pattern is like that of bulk silica 


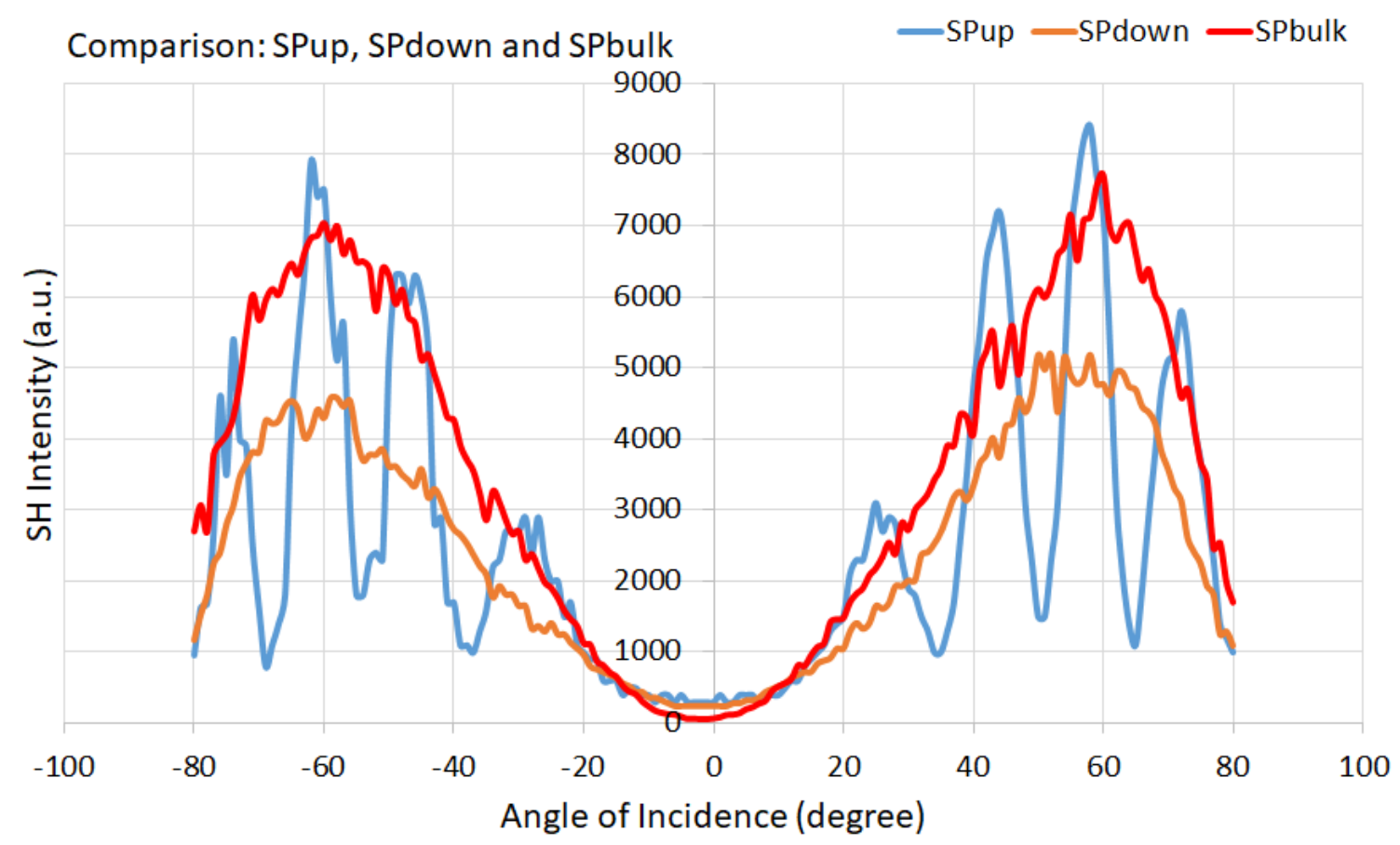

Figure 3.10: Comparison between the SH intensities of the maker fringe patterns of SPup, SPdown and SPbulk

substrate, the weaker SH intensities show that the layers affect the strength of the induced nonlinearity. However, this aspect is subject to verification since the samples SPbulk and SNbulk were not poled at the same time as the multilayer samples.

The subtle aspects discussed in this section may prove crucial for the optimization of the design of multilayer structure. The extent of the induced nonlinearities in the samples will be estimated using the MATLAB simulation model in chapter 4 .

\subsubsection{Reversal of Sample Orientation During Maker Fringe Experiment}

The unique characteristics of the Maker fringe patterns presented in section 3.1 imply that sample orientations play a major role in inducing different types of nonlinearity in the multilayer structures. This phenomenon gives rise to a new question. Will altering the sample orientation during the Maker fringe experiment help extract further information, e.g. locations of the nonlinear regions, structural symmetry or 


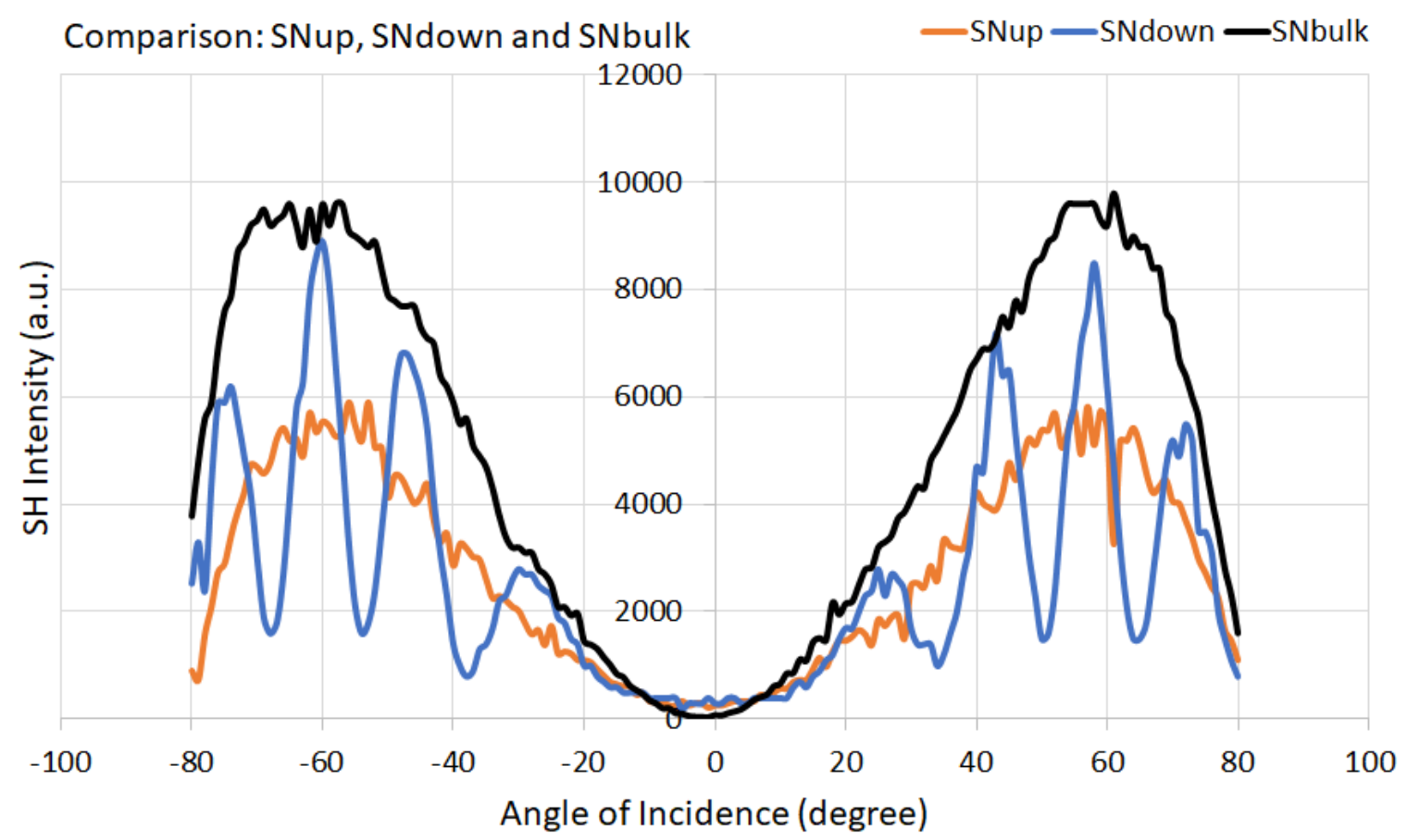

Figure 3.11: Comparison between the $\mathrm{SH}$ intensities of the maker fringe patterns of SNup, SNdown and SNbulk

fabrication defects?

Figure 3.12 shows the two orientations of each sample during the Maker fringe experiments. In Figure 3.12a, the multilayer stack is facing the incoming beam. In Figure $3.12 \mathrm{~b}$, the sample turned around $180^{\circ}$ so that the substrate is facing the incoming beam. The resulting Maker fringe patterns from this investigation is discussed below.

Figure 3.13 shows a comparison between the Maker fringe patterns obtained from the sample SPup. No significant difference is noticeable from this graph. Likewise, Figure 3.14, 3.15, and 3.16 show the Maker fringe patterns obtained from samples SPdown, SNup, and SNdown, respectively. Apart from some small experimental anomalies, none of the graphs show any notable differences between the patterns.

From these observations, it seems that the Maker fringe patterns remain unaffected by altering the sample orientations during the Maker fringe experiments. However, in 


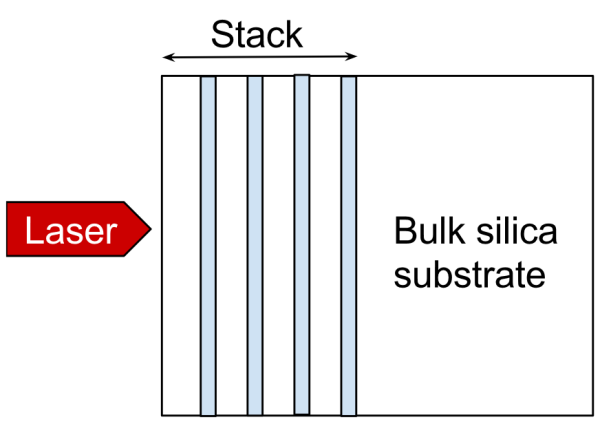

(a) Multilayer stack facing beam

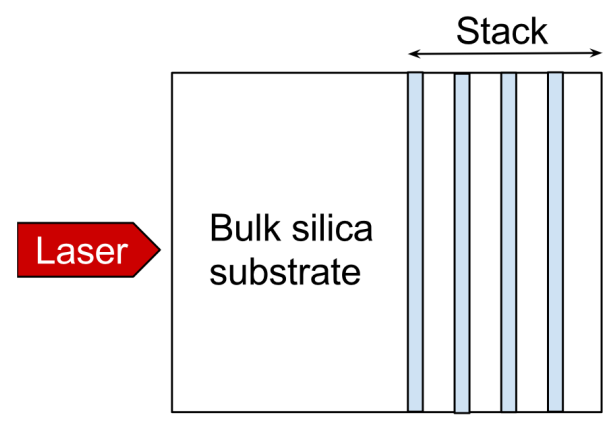

(b) Multilayer stack facing opposite to beam

Figure 3.12: Alternate orientations of the samples during Maker fringe characterization

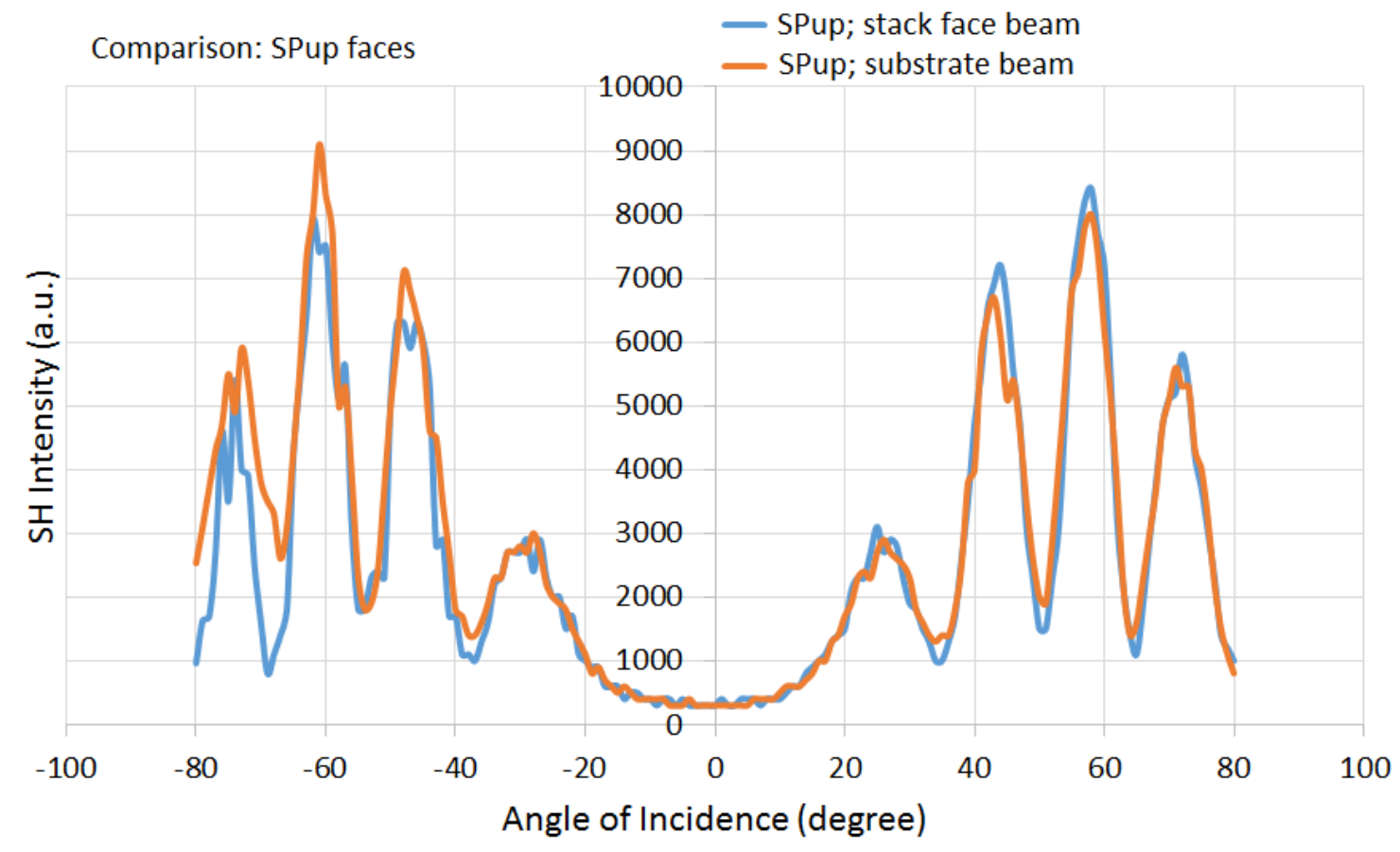

Figure 3.13: Comparisons between Maker fringe pattern from the two faces of SPup.

this experimental setup, for a focusing lens of $15 \mathrm{~cm}$, aperture diameter of $10 \mathrm{~mm}$ and a fundamental wavelength of $1064 \mathrm{~nm}$, the Rayleigh length is $\sim 2400 \mu \mathrm{m}$ [34]. This length is considerably larger than the thickness of the thin film samples $(\sim 500 \mu \mathrm{m})$. It is likely that the samples are too thin relative to the beam focus, thus becoming effectively symmetric from both faces. A tighter beam focus with a shorter Rayleigh length will increase the resolution and may reveal useful information regarding nonlinearity profile. This aspect is left to be investigated further in another study. 


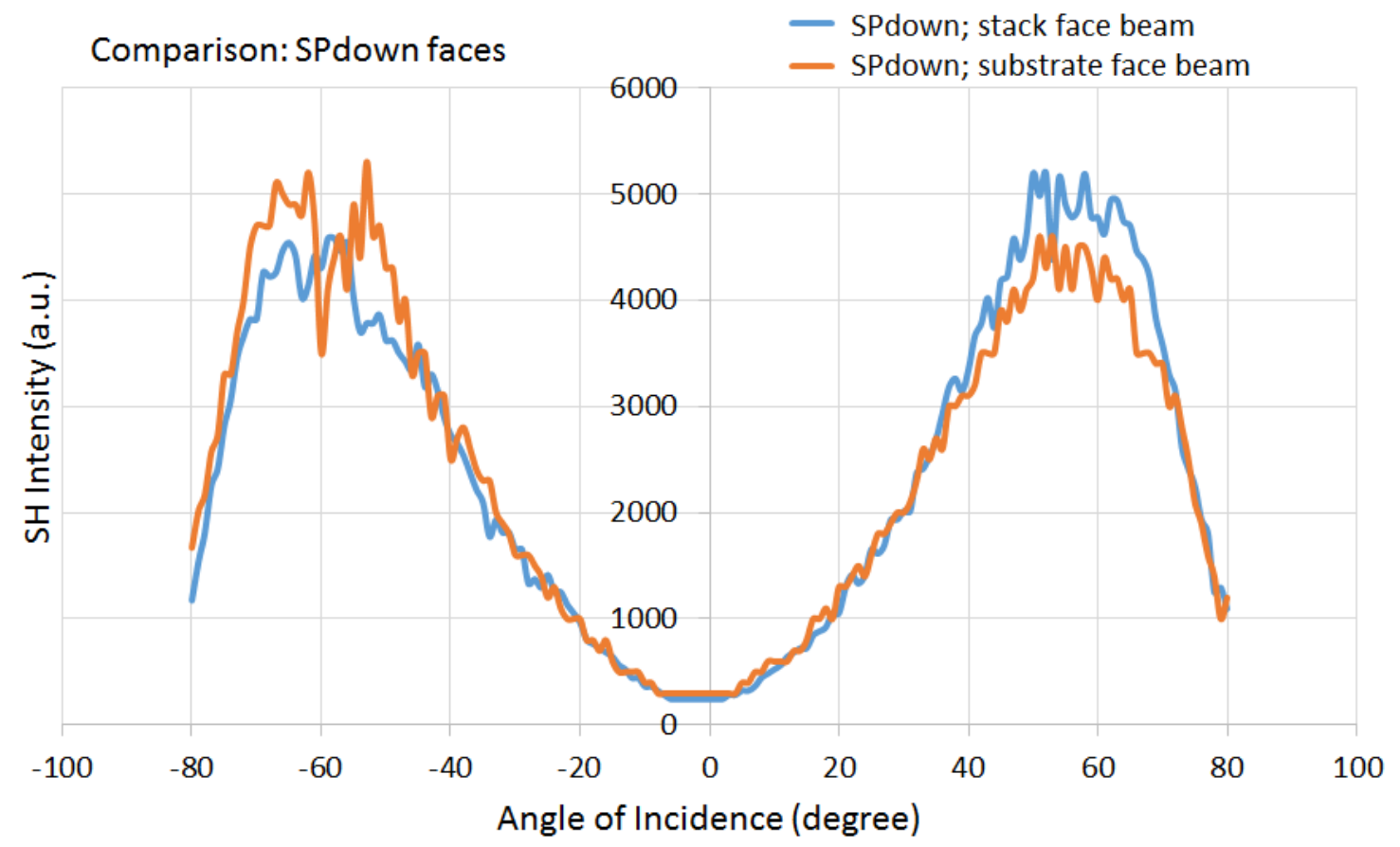

Figure 3.14: Comparisons between Maker fringe pattern from the two faces of SPdown.

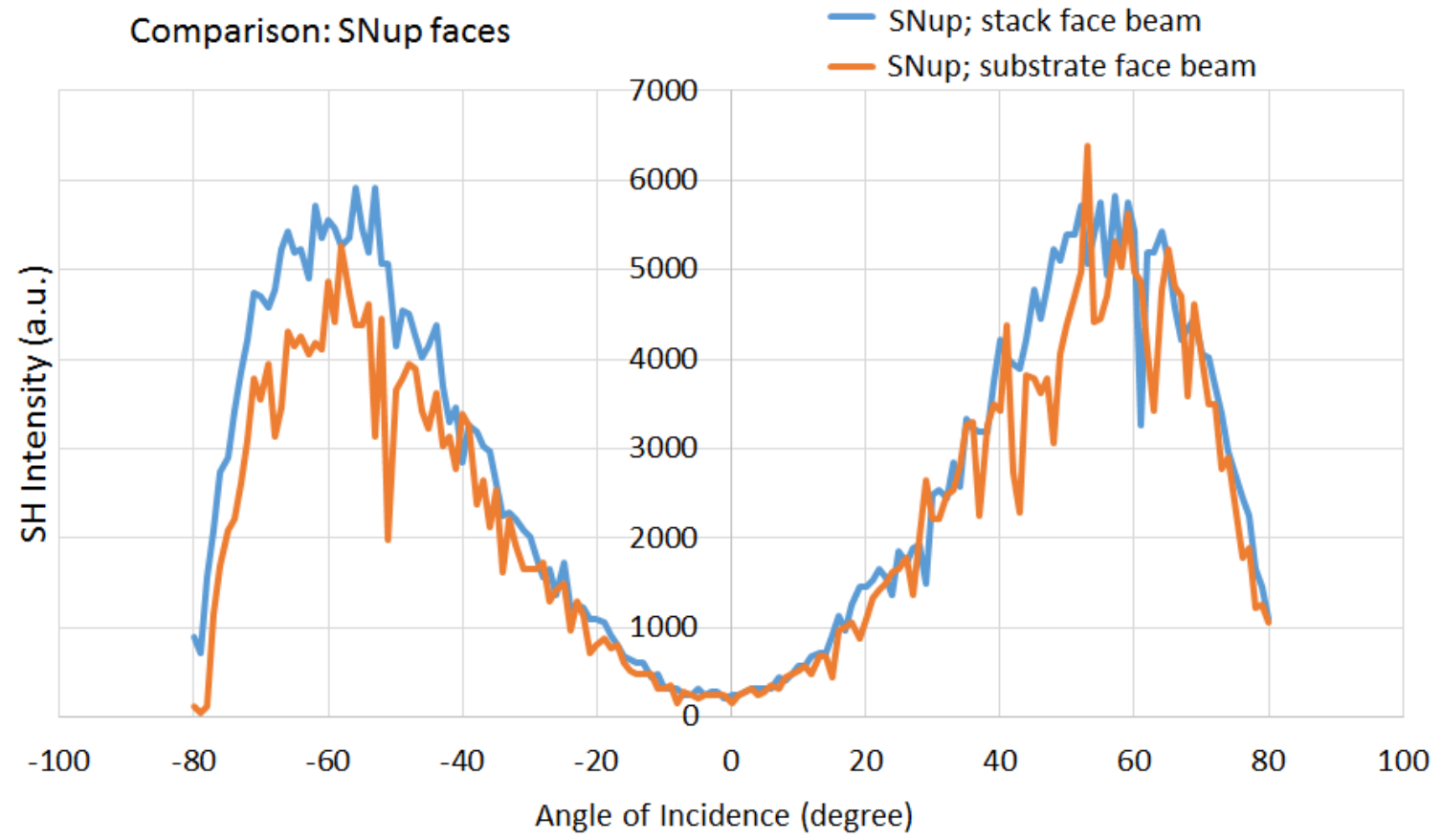

Figure 3.15: Comparisons between Maker fringe pattern from the two faces of SNup. 


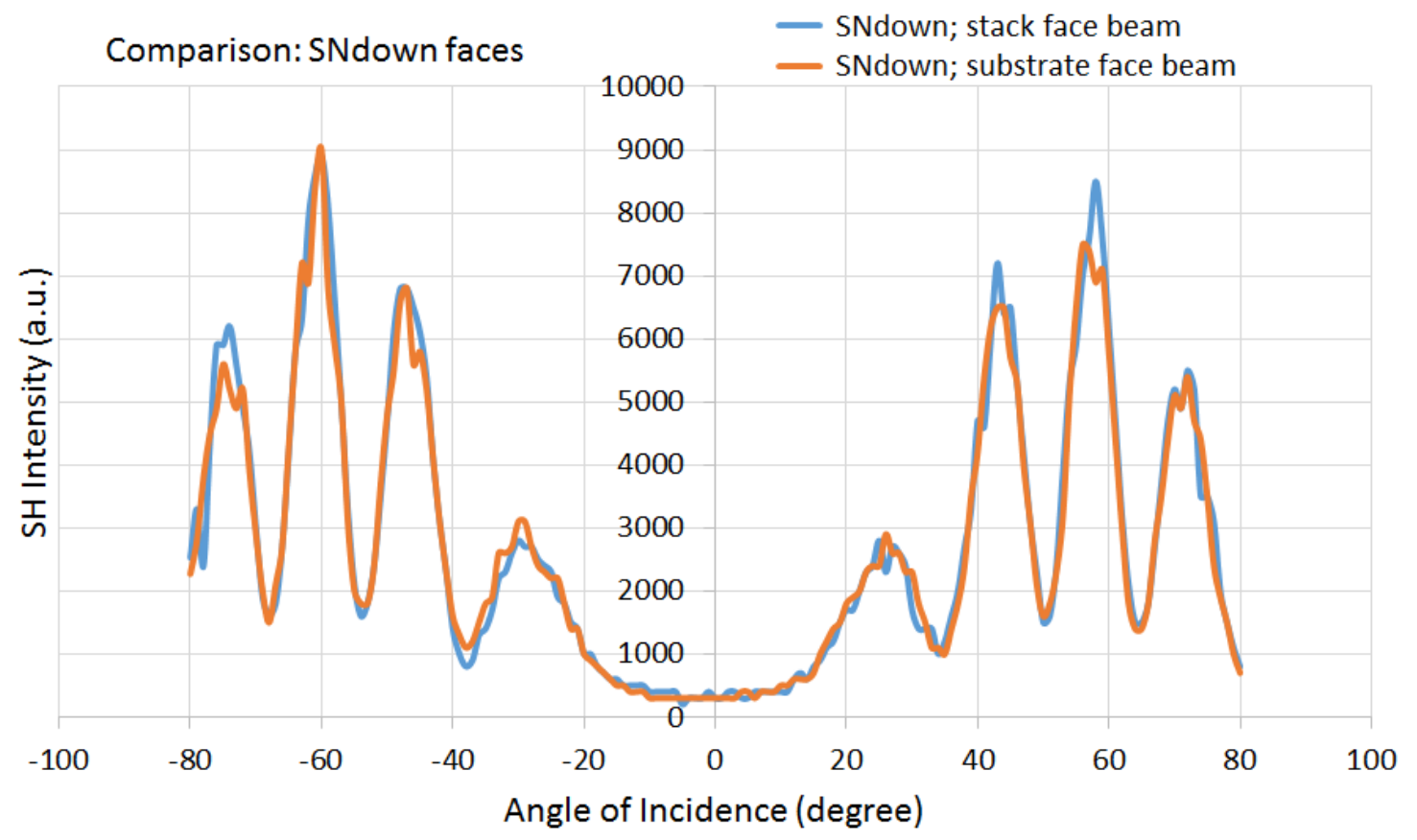

Figure 3.16: Comparisons between Maker fringe pattern from the two faces of SNdown.

\subsection{Reproducibility of Results}

This section presents a study of multilayer structures that test the reproducibility and variability of the modulation fringes in the Maker fringe patterns shown earlier in section 3.1. This investigation can ensure that the unique artifacts are not due to any random fabrication or experimental anomaly. Therefore, in the next set of experiments, some of the experimental parameters were varied, to observe the consistency of the induced nonlinearity in the samples.

\subsubsection{Variation of Laser Beam Power}

The general relationship between the powers of the input pump through a nonlinear sample and the corresponding SH signal is $I_{2 \omega} \propto I_{\omega}^{2}$. This section presents a study to test the multilayer structures for compliance with this relationship. The sample SNup is investigated in this set of experiments.

The average SH power over five data points, from -30 to $-34^{\circ}$ is plotted against the input power (five power levels). Figure 3.17 shows the experimental observation (green) alongside a quadratic graph (gray). The graph from the experiment has been 
fitted with absolute error bars of \pm 2400 a.u. and $\pm 20.25 \mathrm{~mW}$ for the SH intensity and the input pump power, respectively. A discussion regarding the measurement errors is provided in section 2.3.

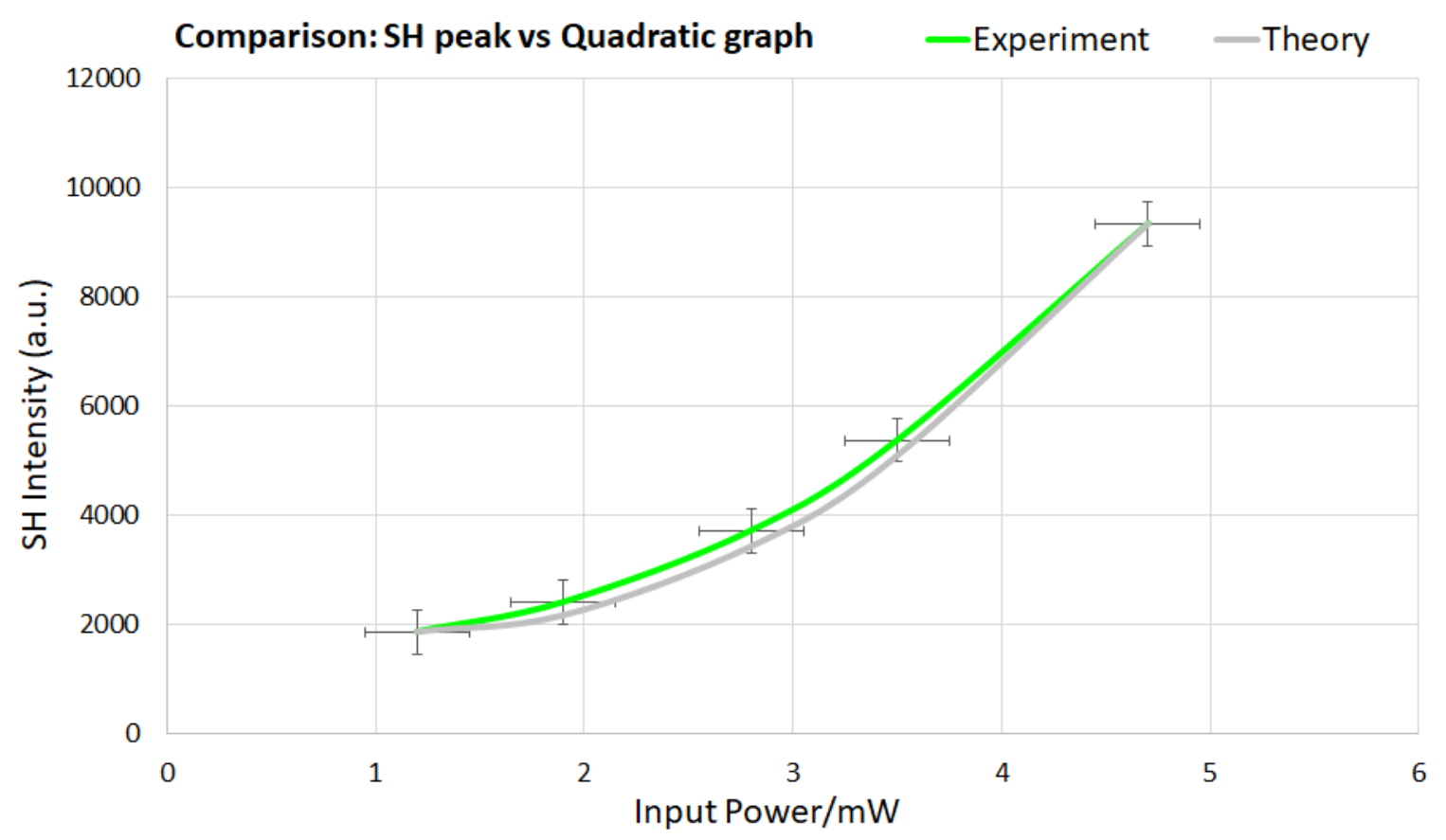

Figure 3.17: Graph showing the applicability of the relationship $I_{2 \omega} \propto I_{\omega}^{2}$ for the multilayer structures

It may be worthwhile to investigate the relationship more accurately for the multilayer silica structures. The Maker fringe setup used in this project is limited to a rather narrow range of input power. It was not possible to measure power levels of the incoming beam below $1 \mathrm{~mW}$ with the power meter, which resulted in the graph shifting away from the y-axis. For power levels beyond $5 \mathrm{~mW}$, the PMT for detecting the SH signal gets saturated. The PMT also records background noise at a zero input pump, which could be the reason for the graph to show an $\mathrm{SH}$ intensity at $0 \mathrm{~mW}$ (upon extrapolation). Therefore, one significant improvement in the experimental setup would be to widen the range of applicable input power.

The best fit curve can be drawn by first shifting the graph to the origin, which presumably counteracts the limitations mentioned above. The resulting equation is: 


$$
y=610 x^{2}+\left(7 \times 10^{-12}\right) x-\left(7 \times 10^{-12}\right)
$$

The second and third terms in equation 3.1 are small and thus negligible. The remaining part of the equation suggests that the relationship $I_{2 \omega} \propto I_{\omega}^{2}$ holds for the multilayer structures.

\subsubsection{Effect of Aging on Induced Nonlinearity}

In this set of experiments, the Maker fringe characterizations were performed on individual samples, multiple times with varying time intervals, starting from one day to approximately three months. This study gives an idea of the effect of aging on the modulation fringes observed in the Maker fringe patterns. It can also help detect any bias during a single Maker fringe experiment. Any bias identified would be eliminated to ensure consistency across all the experiments.

Figure 3.18 shows two Maker fringe patterns, obtained from sample SPup. The time duration between the two measurements is $\sim 1.5$ hours with an input power less than $1 \mathrm{~mW}$. The sample is one day old, which means that the Maker fringe characterization of the sample took place one day after poling. The graph shows that the patterns are almost identical.

Figure 3.19 shows the Maker fringe patterns obtained from sample SPup, with an input power of $2.1 \mathrm{~mW}$. This time, the blue and orange graphs are for the sample when it is 12 and 23 days old, respectively. The patterns are almost identical, apart from a slight difference in the SH peak, on the right side of the normal.

Another sample from the multilayer structures that produced modulation fringes is SNdown. Figure 3.20 shows its Maker fringe patterns at an input power of $2.1 \mathrm{~mW}$. The blue, orange and green graphs are from the sample when it is 11, 18 and 99 days old, respectively. The Maker fringe patterns are nearly identical except for slight differences in SH peaks. A similar variation was seen in the case of sample SPup (in Figure 3.19). These random variations would be due to experimental anomalies rather than a real difference. 


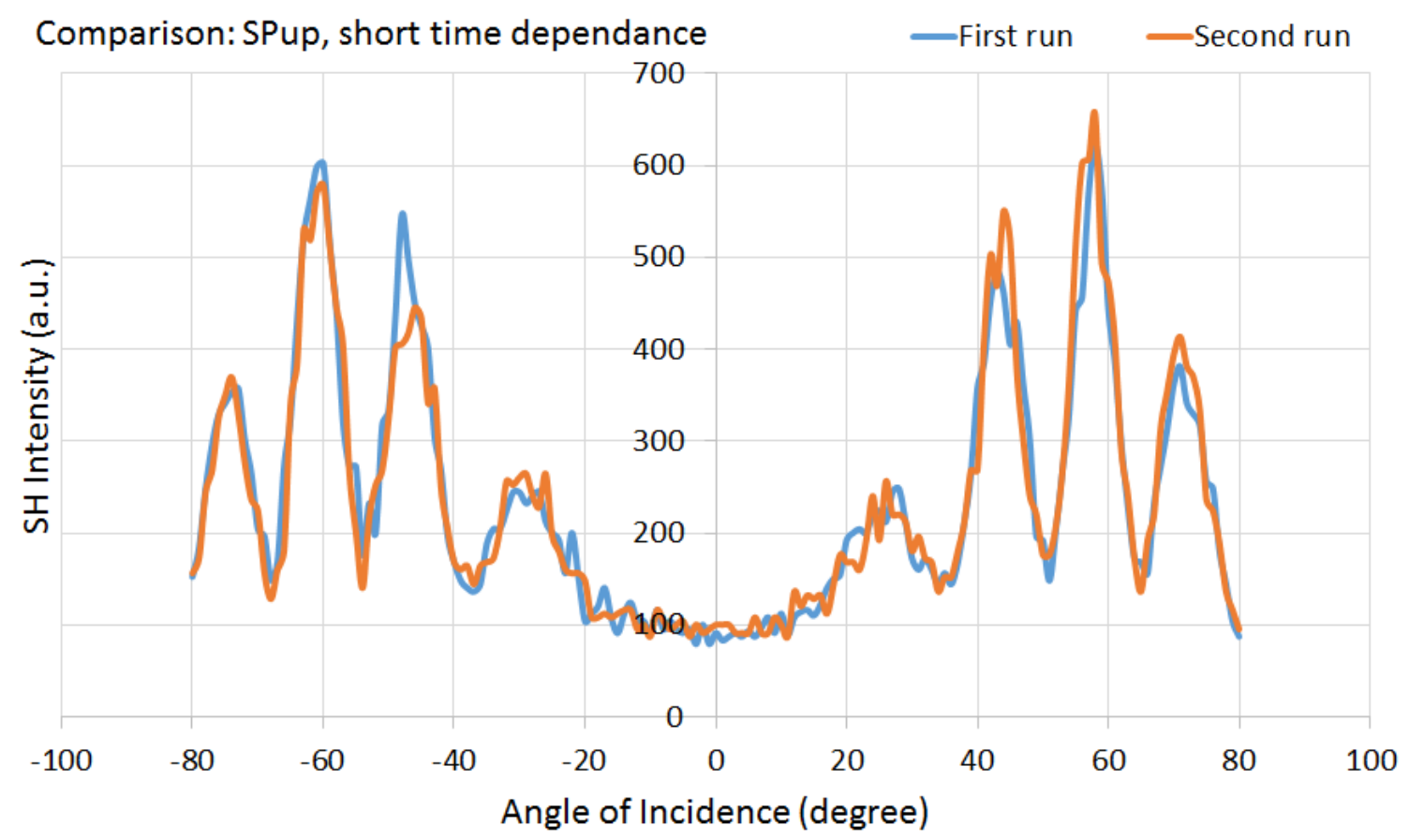

Figure 3.18: Comparison between Maker fringe patterns from repeated measurements of sample SPup, input power $<1 \mathrm{~mW}$.

The observations from these sets of Maker fringe patterns provide further confirmation that the artifacts of destructive and constructive interferences are unique to the multilayer structures. It also suggests that the aging of the samples does not affect the induced nonlinearity. In fact, this observation is consistent with a previous report, where the nonlinearity of silica glass was found to be stable and permanent for months [8]. In the study, characterization using etching revealed that, after as long as 14 months, the remaining depletion region generates a lower, but constant $\mathrm{SH}$, which implies that the poling process causes a permanent alteration in the molecular structure of silica.

\subsubsection{Phosphorous Doped Multilayer Structure}

Among the biggest contributing factors affecting the properties of a sample is its fabrication process. Therefore, for this set of experiments, an entirely new sample is fabricated with phosphorous replacing germanium as dopants in the alternating doped layers. All the rest of the specifications are same, as was shown in Figure 2.1. The time difference between the experimental runs of the phosphorus and germanium 


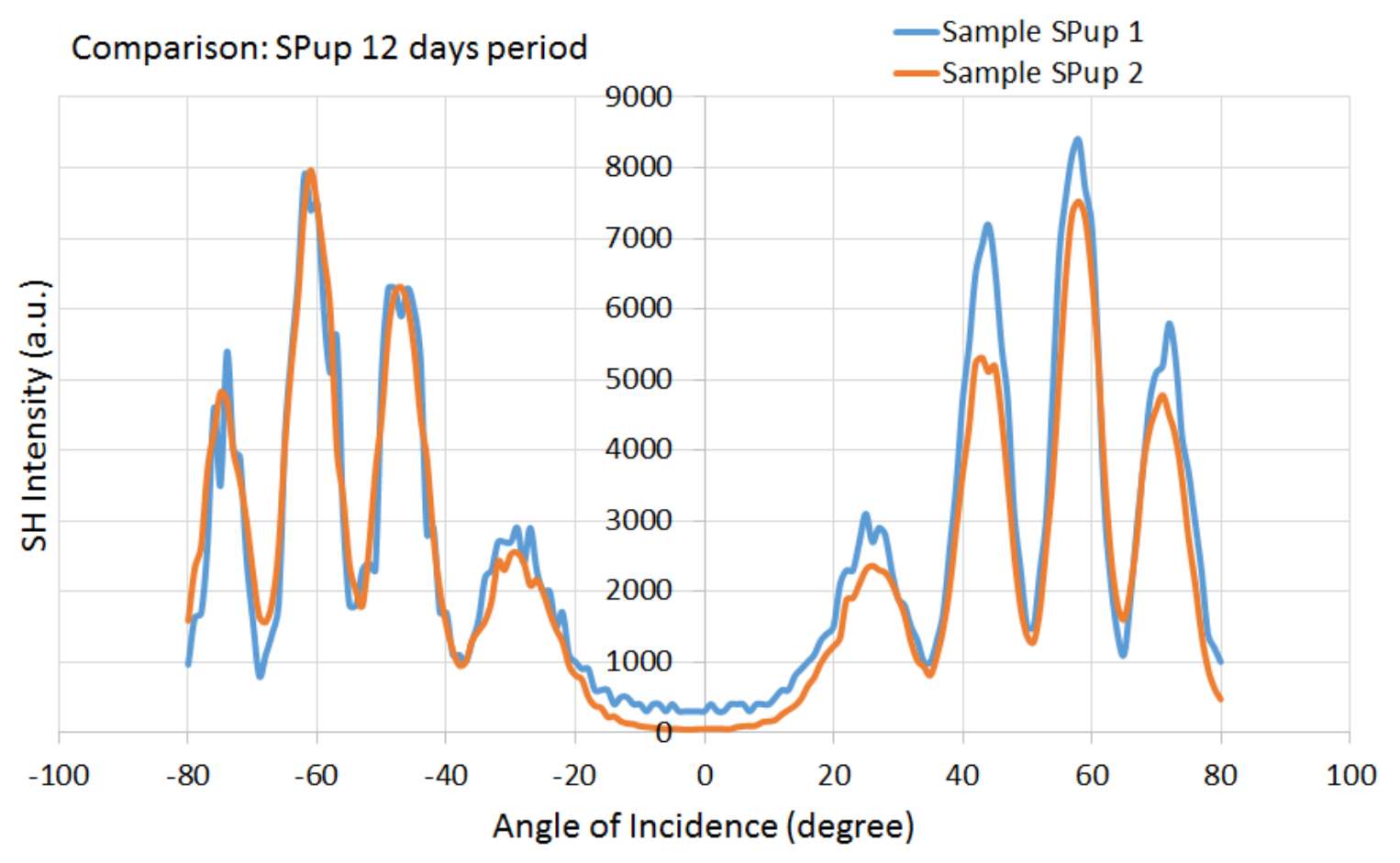

Figure 3.19: Comparison between Maker fringe patterns from experiments 12 days apart (sample SPup).

doped samples is approximately four months.

Figure 3.21 shows the Maker fringe pattern obtained from a positively poled sample (with layers stack facing the needle). Modulation fringes have appeared like the case of the germanium doped samples. However, the fringes are not as deep which suggests that the induced nonlinearity is different for phosphorous doped multilayer structures.

Figure 3.22 shows the Maker fringe pattern obtained from a negatively poled sample (with layers stack facing the needle). This case shows no modulation fringes, as was seen in the germanium doped samples (sample SNup).

\section{Molecular Structures of Germanium and Phosphorus dopants}

Phosphorus integrates differently than germanium as dopants in silica glass, which may be the reason for the differences in the induced nonlinearity (as suggested by the Maker fringe patterns) between the two types of multilayer structures. This section presents a brief discussion of the molecular structure of germanium and phosphorus 


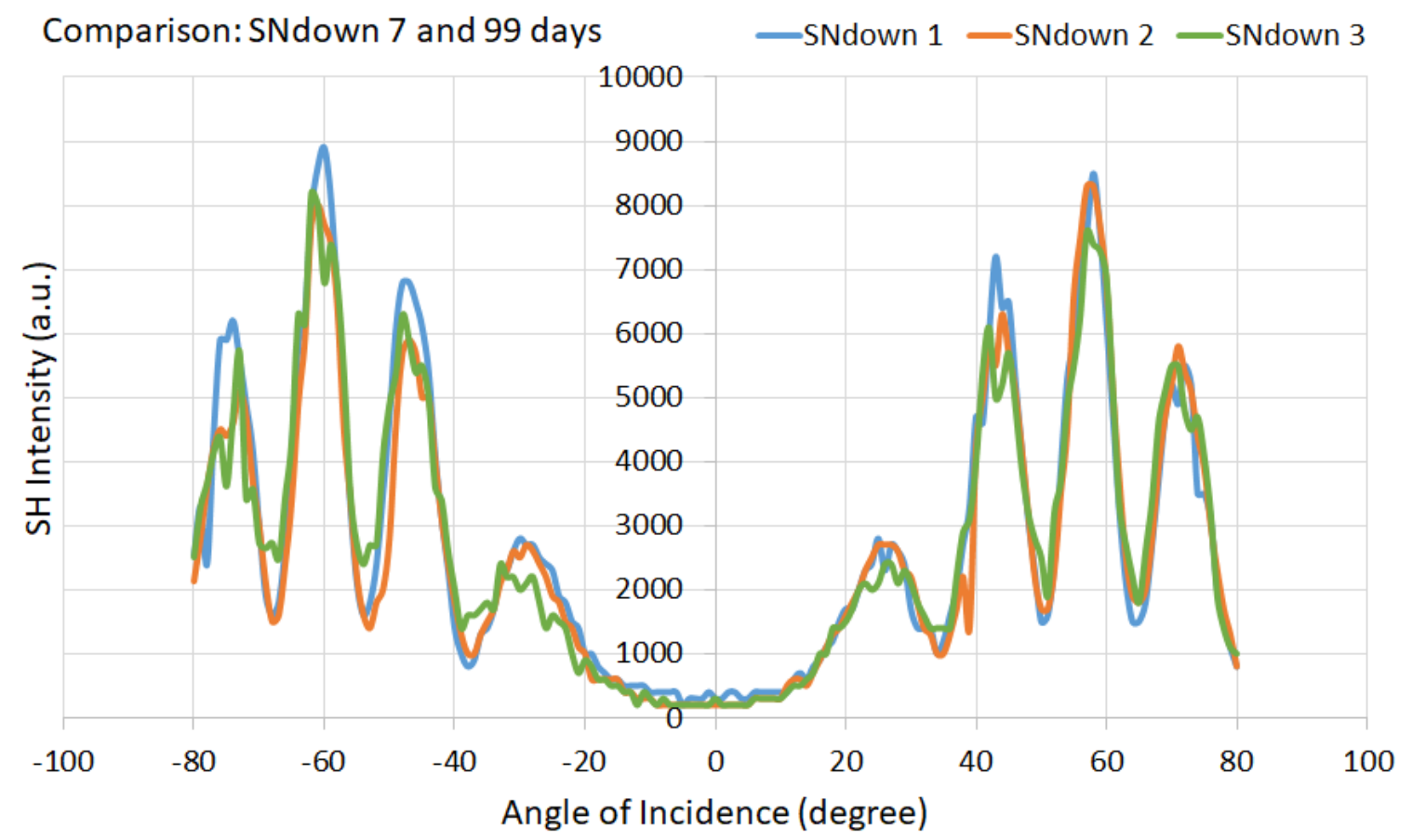

Figure 3.20: Comparison between Maker fringe patterns from experiments 7 days apart (sample SNdown).

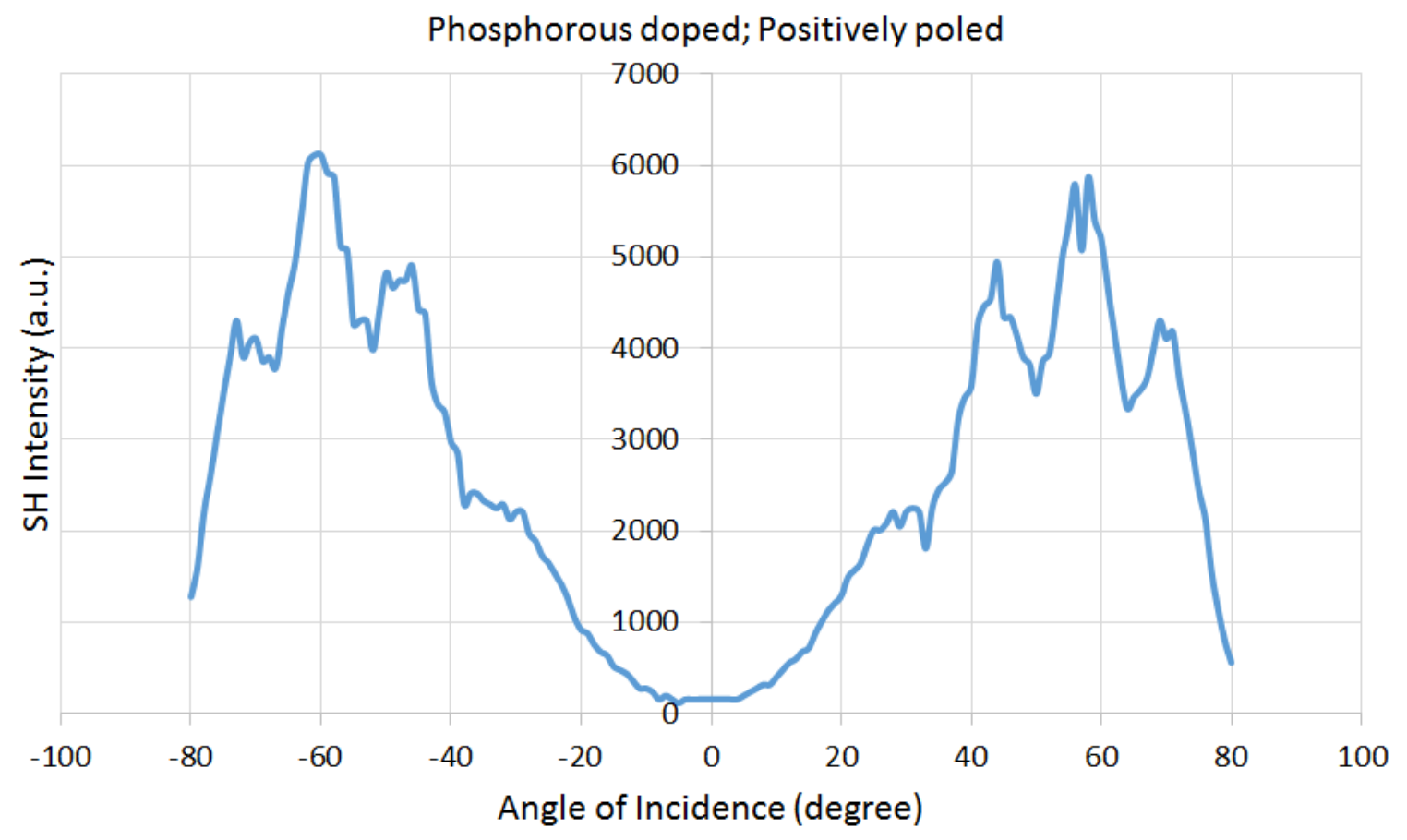

Figure 3.21: Maker fringe patterns from positively poled phosphorous doped sample. 


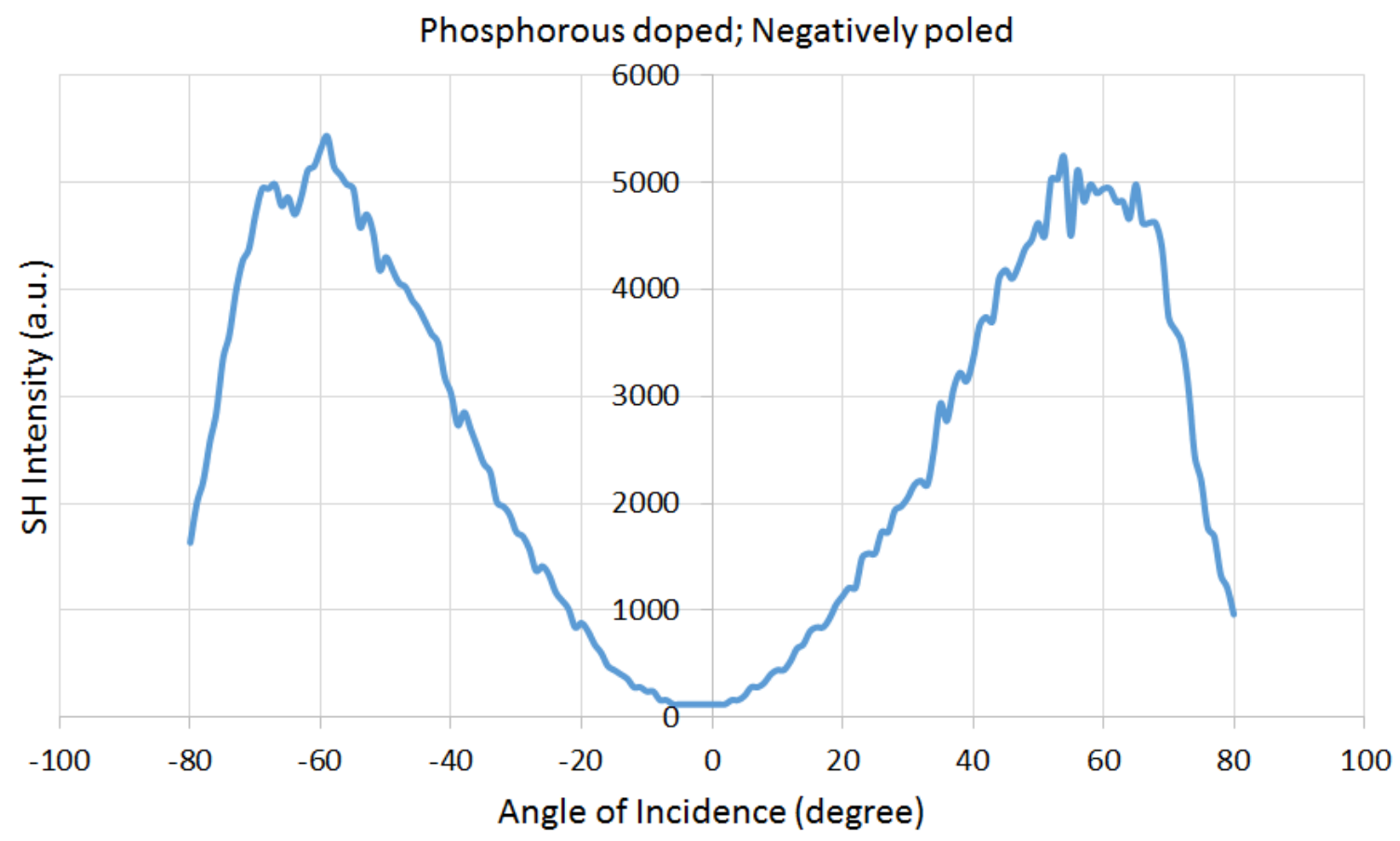

Figure 3.22: Maker fringe patterns from negatively poled phosphorous doped sample.

doped silica.

Germanium has four valence electrons like silicon. It replaces $\mathrm{Si}^{4+}$ in the silica matrix without any charge compensating ions [45]. Experimental evidence demonstrated the charge blocking capability of the interface between germanium doped and undoped silica layers [37]. This blocking effect causes migrating positive charges to accumulate at the interfaces to create strong localized nonlinearity peaks. Another important factor about doping silica glass with germanium is that it raises the refractive index, which in turn raises $\chi^{(3)}$. And according to equation 1.1, the effective $\chi^{(2)}$ also increases. The refractive index of germanium doped silica glass increases further during the poling process [46].

On the other hand, phosphorus has five valence electrons. It incorporates into the silica lattice by forming the pentavalent oxide, $\mathrm{P}_{2} \mathrm{O}_{5}$ [47], as shown in Figure 3.23. There is one extra electron that incorporates with a nonbridging oxygen ion $\left(\mathrm{O}^{-}\right)$. Since the nonbridging oxygen ion has an affinity towards positive charges, it can effectively trap the migrating positive charges via Coulomb interaction, thus raising the effective $\chi^{(2)}$. 


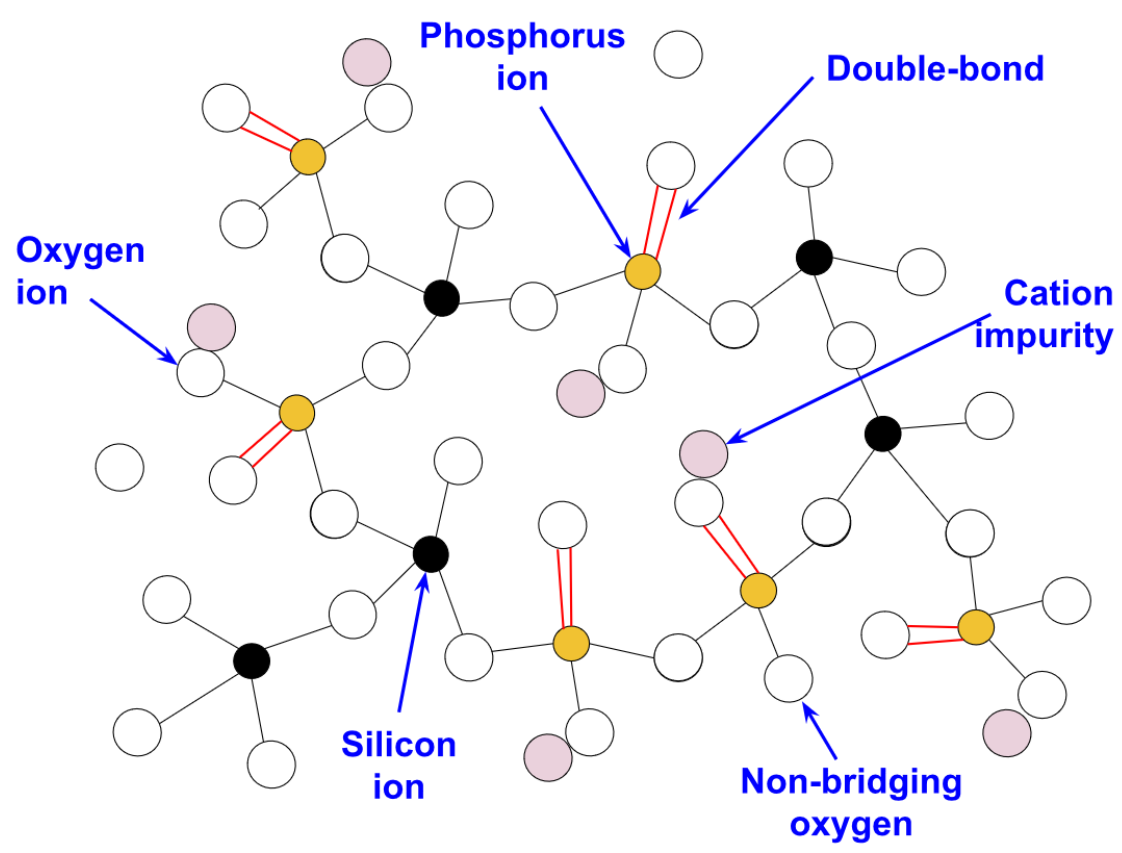

Figure 3.23: $\mathrm{P}_{2} \mathrm{O}_{5}$ formation in silica matrix

The results presented in this section strongly indicate that the modulation fringes in the Maker fringe patterns are unique to the multilayer silica structures. It is particularly interesting to see the contrast between the induced nonlinearities in the multilayer structures of germanium and phosphorus doped samples. A detailed study of molecular structures of the dopants and the corresponding nonlinearity induced in silica glass is essential, however outside the realm of this research project. It is also important to investigate the dependence of all the other experimental parameters on the induced nonlinearity. Further study into this aspect can lead to the discovery of powerful ways to optimize the design of the multilayered silica structures. 


\section{Chapter 4}

\section{Matlab Simulation}

This chapter presents a MATLAB simulation model originally developed by Nasr et al. [48], to determine the distribution of the induced nonlinearity in poled multilayer structures. The MATLAB model has been modified for this research project to not include simulation with split step Fourier transforms, which deals with the walk-off effect of the fundamental and the SH waves for the case of femtosecond pulses. This research project uses a picosecond pulse source to characterize the samples.

The simulation model is based on equation (4.1),

$$
\frac{\partial A_{2}}{\partial z}=-\frac{i \omega}{n_{2 \omega} c} d_{e f f} A_{1} \underbrace{A_{1} e^{i \Delta k z}}_{\begin{array}{c}
\text { amplitude } \\
\text { varying } \\
\text { in space }
\end{array}}
$$

where $A_{2}$ is the electric field amplitude of the $\mathrm{SH}$ wave, $A_{1}$ is the electric field amplitude of the fundamental wave, $\omega$ is the angular frequency of the fundamental wave, $n_{2 \omega}$ is the refractive index of the medium at the $\mathrm{SH}$ wavelength, $c$ is the speed of light, $\Delta k$ is the wavenumber mismatch term, $z$ is the propagation distance in the nonlinear material, and $d_{\text {eff }}$ is expressed as:

$$
d_{\text {eff }}=d_{33} \sin (\theta)
$$

where $d_{33}$ is an element of the SON susceptibility coefficient matrix $\left(d_{i l}\right)$, and $\theta$ is the angle of incidence of the beam on the sample 


\subsection{Numerical Estimate of Induced Nonlinearity}

In the MATLAB model, the nonlinearity profiles are simulated such that the resulting Maker fringe patterns resemble those obtained experimentally (shown in section 3.1). In this way, the properties of the actual nonlinearity profiles, i.e. width and depth, are estimated.

\subsubsection{Maker Fringe Pattern with Modulation Fringes}

Figure 4.1a shows a simulated Maker fringe pattern that resembles the experimental patterns shown previously in Figure 3.8 (reproduced in Figure 4.1b). According to the simulation, the nonlinearity profile consists of two thin nonlinear regions separated by a distance of $460 \mu \mathrm{m}$. The first region has a width of $5 \mu \mathrm{m}$ and lies at the stack of layers. The other region is located inside sample and has a width of $5 \mu \mathrm{m}$ as well. Figure 4.3a and Figure 4.3d depicts the corresponding nonlinearity profile for the samples SPup and SNdown, respectively.

One can note that according to the MATLAB hypothesis, the second nonlinear layer is $34.8 \mu \mathrm{m}$ away from the surface of the sample (total sample width: $504.8 \mu \mathrm{m}$ ). This theoretical observation may not be accurate, and in reality, the two nonlinear layers may simply be near the surfaces. Several factors may be the cause of this discrepancy. Firstly, the hypothesis does not account for the expected rise in the refractive index of the samples due to poling, as well as the introduction of dopants [46]. Rather, the general refractive index of fused silica is used, which are 1.4496 and 1.4607 for the fundamental and SHG waves, respectively [49]. Secondly, the manufacture's statement of the sample width is assumed to be accurate in the hypothesis. It would be ideal to re-measure the width of the samples, especially after the fabrication stage.

\subsubsection{Maker Fringe Pattern without Modulation Fringes}

In section 3.2.2, it was shown that the Maker fringe patterns of the multilayer samples with no modulation fringes were $\sim 65 \%$ of the ones with modulation fringes. Figure 4.2a shows such a Maker fringe pattern obtained in simulation (Figure 3.9 reproduced here in Figure 4.2b for ease of comparison). The SH peak is above $6 \times$ $10^{5}$ a.u., which is $\sim 65 \%$ of the SH peak of the modulation fringe in Figure 4.1a. This nonlinearity profile consists of a single nonlinear region of width $\sim 9 \mu \mathrm{m}$, as 
shown in Figure 4.3b and Figure 4.3c for the samples SPdown and SNup, respectively. According to the charge migration model discussed in section 1.3, the nonlinear layer would form near the anode.

Figure 4.3 shows all the simulated nonlinearity profiles of the poled multilayer structures, labeled with the width of nonlinear regions and the depth of their locations.

The MATLAB model presented in this chapter provides only a hypothesis regarding the extent of the induced nonlinearity of the poled multilayer structures. Experimental techniques like SH microscopy, etching or layer peeling needs to be used to verify these findings. It is imperative to investigate the multilayer structures further to obtain information on some of the main features of the nonlinearity profile, such as distribution, shape, size and most importantly, the actual mechanism of charge migration. 


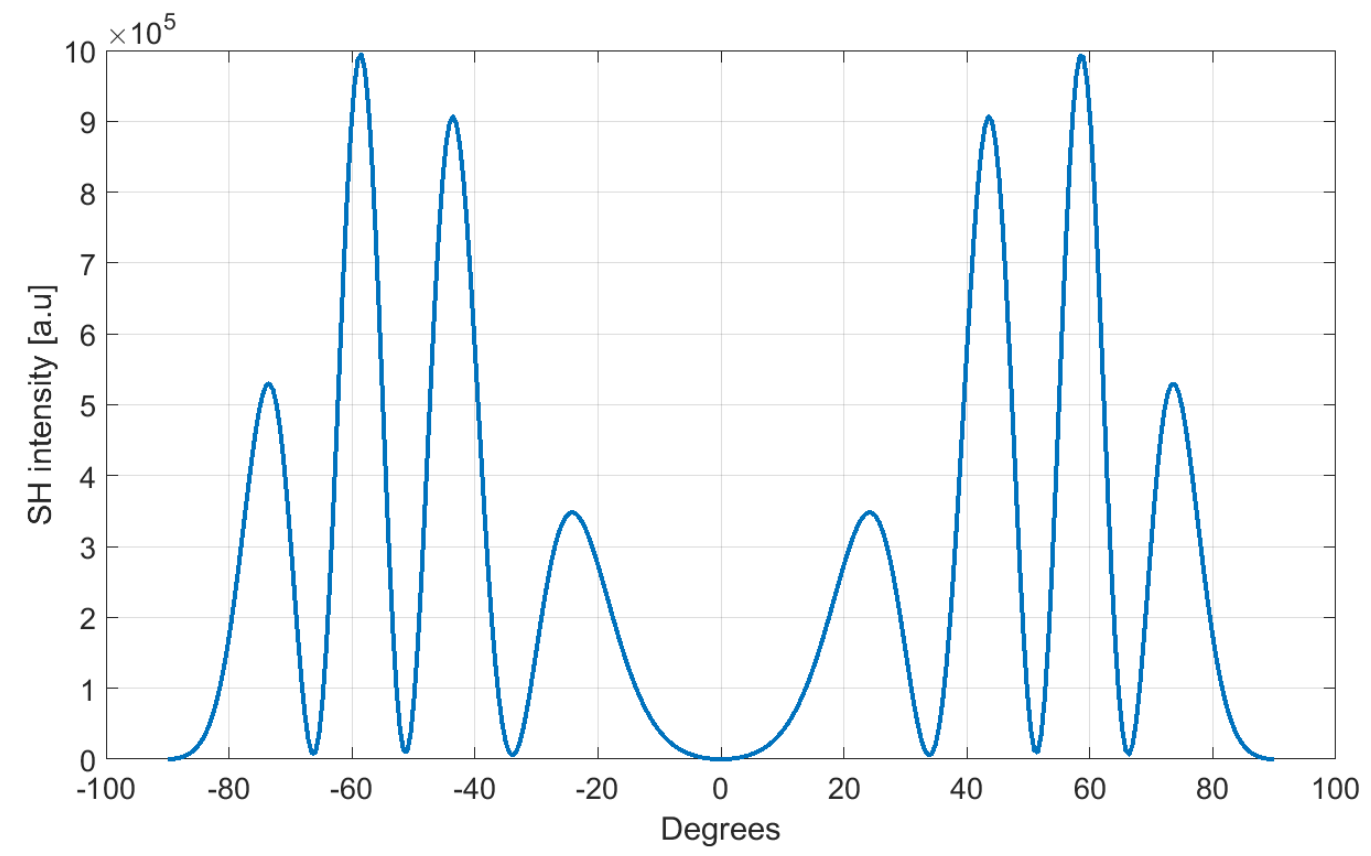

(a) MATLAB simulation for multilayer structures SPup and SNdown

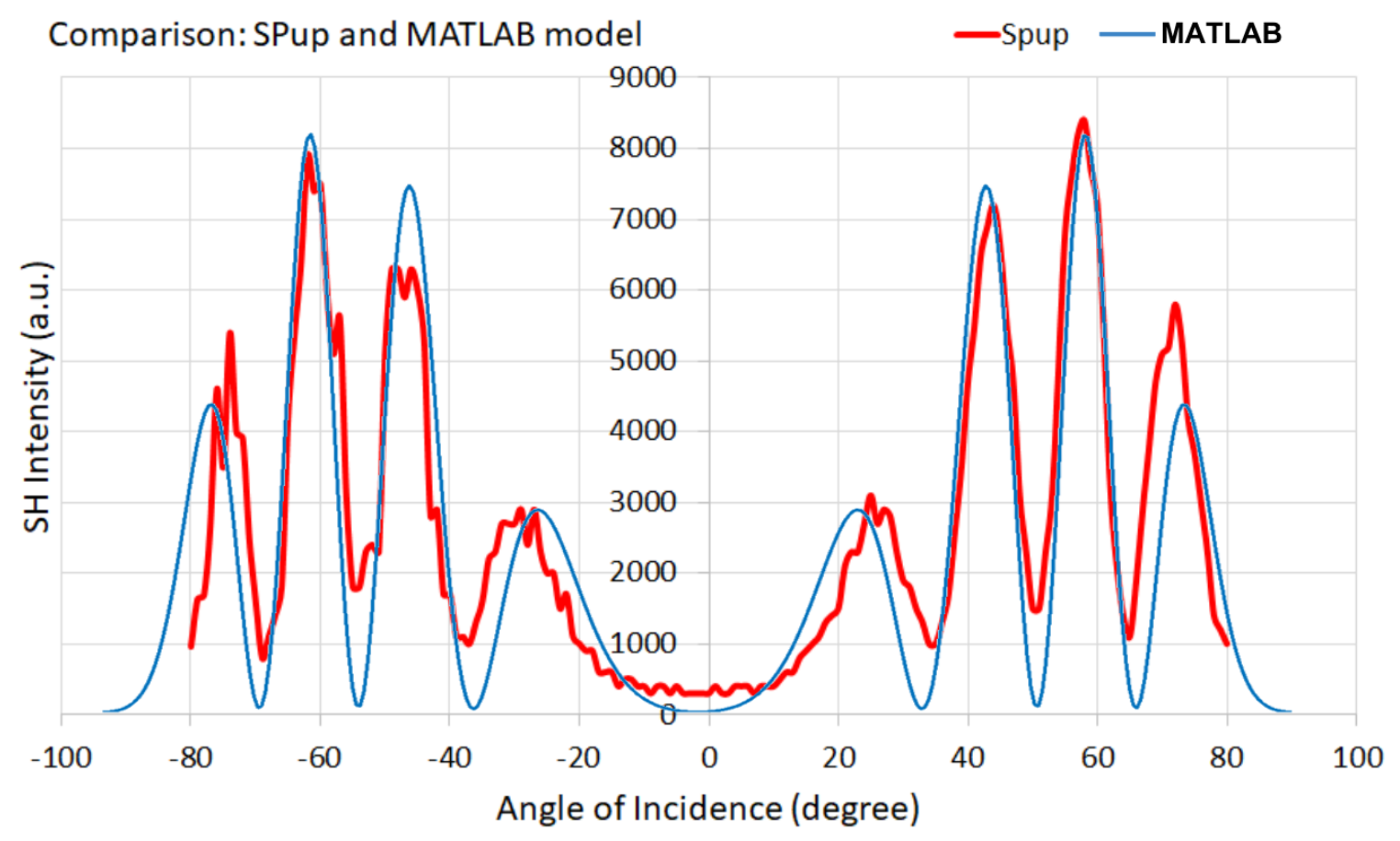

(b) Superposed MATLAB graph on the Maker fringe pattern of SPup

Figure 4.1: MATLAB simulation vs. experimental Maker fringe patterns for samples SPup and SNdown 


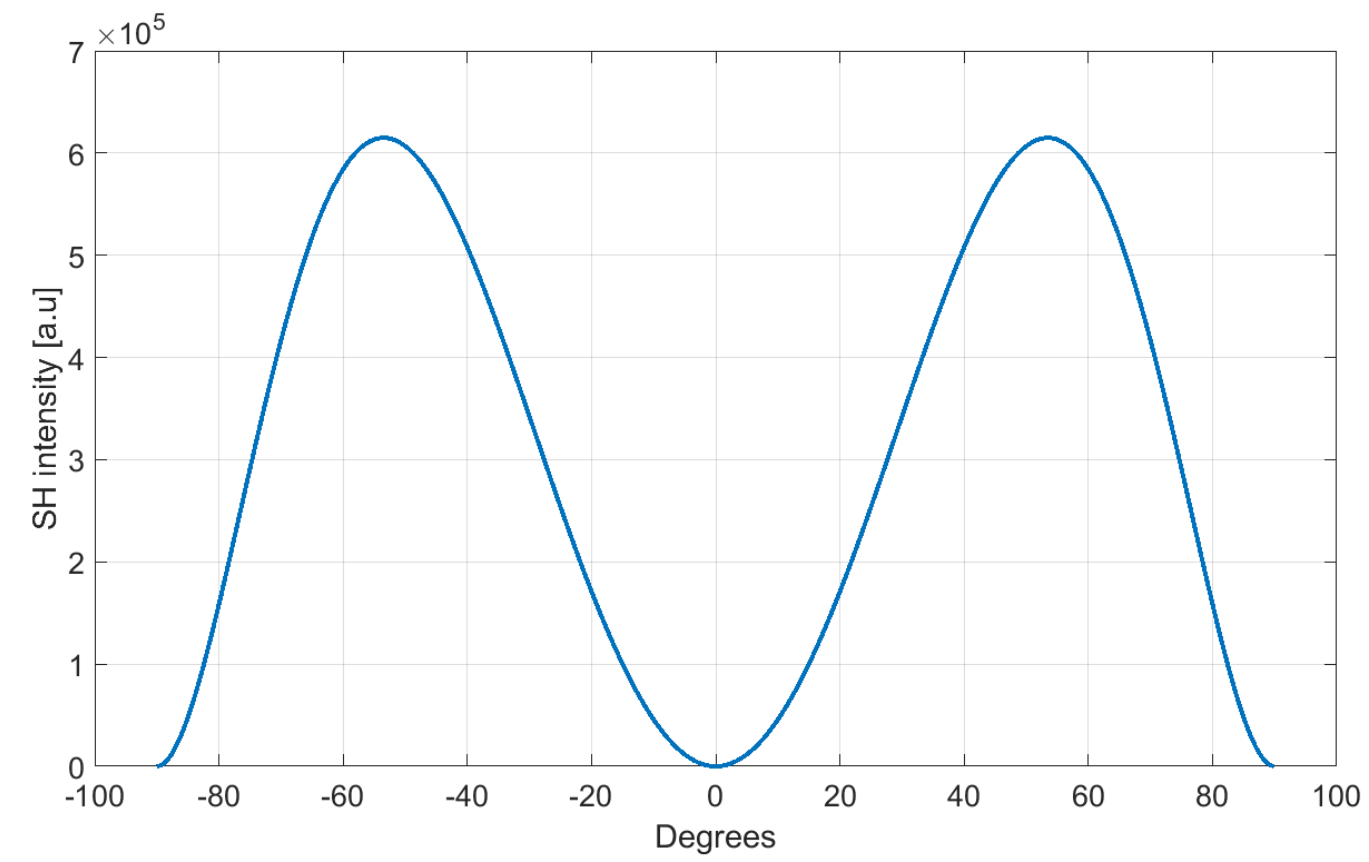

(a) MATLAB simulation for multilayer structures SPdown and SNup

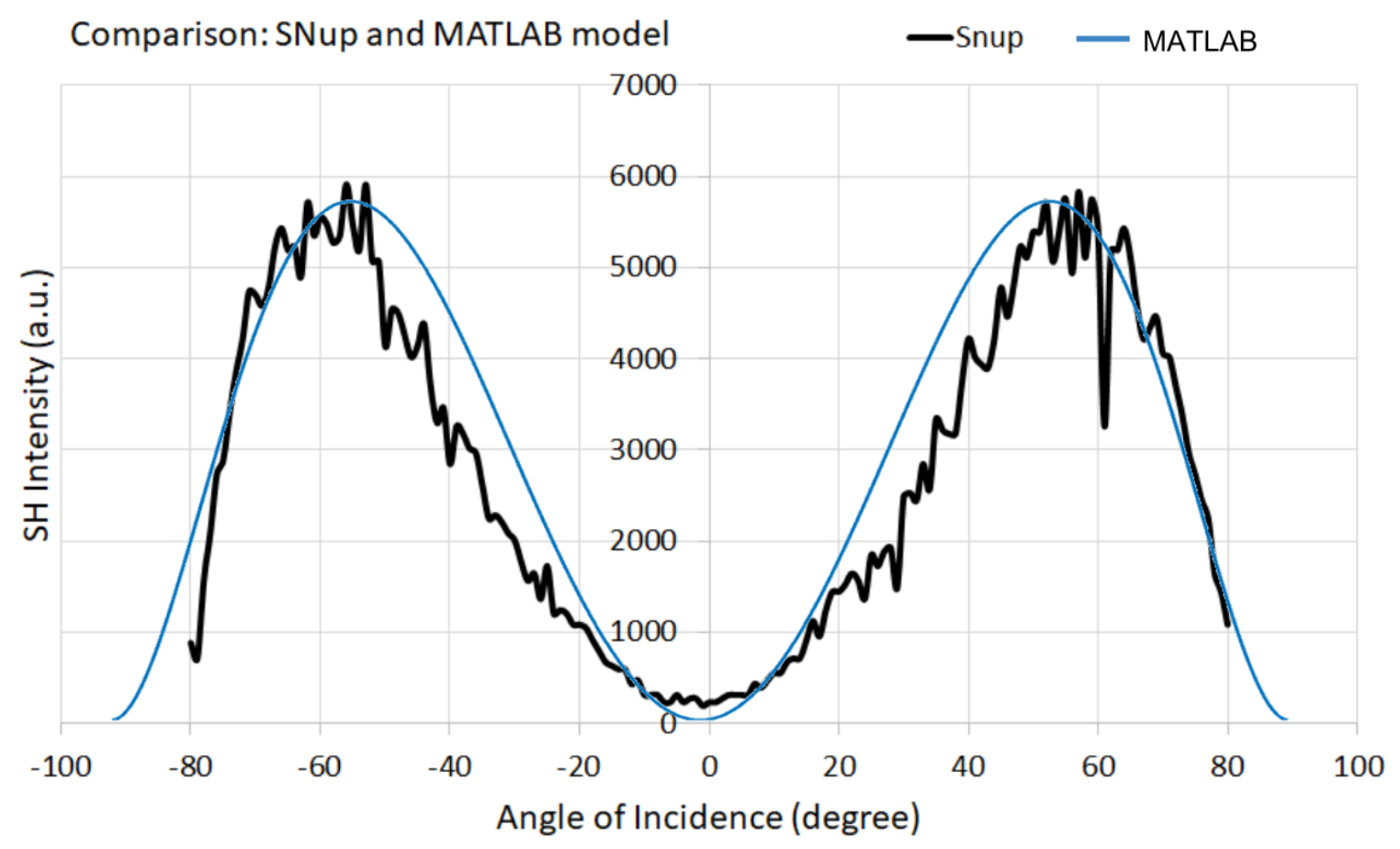

(b) Superposed MATLAB graph on the Maker fringe pattern of SNup

Figure 4.2: MATLAB simulation vs. experimental Maker fringe patterns for samples SPdown and SNup 


$$
+
$$

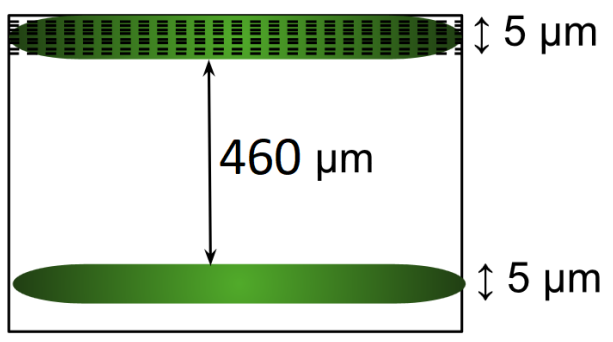

(a) Model for SPup<smiles>C=CC</smiles>

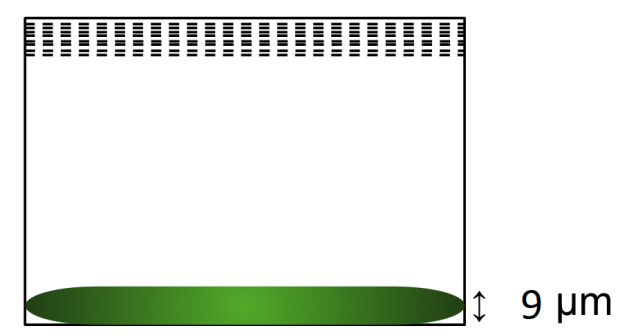

(c) Model for SNup
$+$

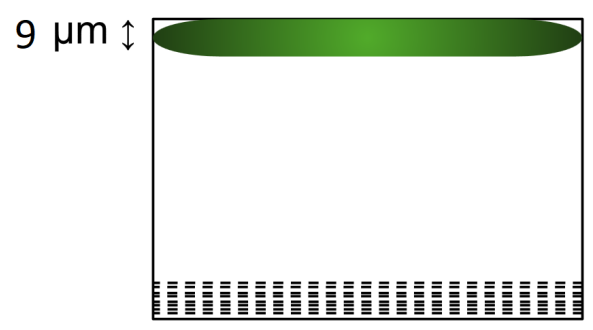

(b) Model for SPdown
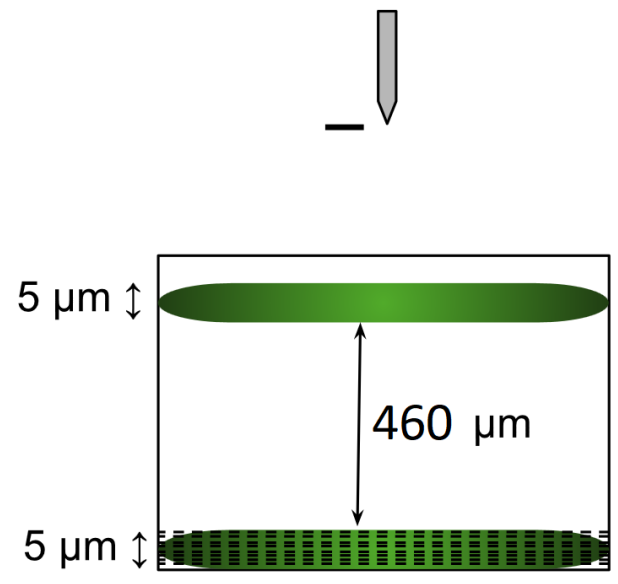

(d) Model for SNdown

Figure 4.3: Simulated charge migration model of the poled samples. The labels indicate the extent and depth of the induced nonlinearity 


\section{Chapter 5}

\section{Summary and Future Work}

This research project studied a model of sub-divided layers of alternating doped and undoped silica, deposited on thin film silica substrates. The purpose of such a multilayer structure is to enhance poling induced nonlinearity in silica glass. The layers introduce interfaces that would block charge migration of impurity alkali ions, thus allowing control over the induced nonlinearity, e.g. extent and location.

During poling, alternation between voltage polarities and sample orientations affect the induced nonlinearity in unique ways. Maker fringe measurements showed modulation fringes in the envelope, for samples that had been poled with the stack of layers facing the anode. When the layers faced the cathode, the Maker fringe patterns resembled that of poled bulk silica, but with significantly lower SH peaks.

Another set of experiments verified that a factor increase in the input pump power resulted in an increase of the SH signal power by the factor squared (with $10 \%$ discrepancy), which abides by theoretical predictions. The poling effect was stable for more than three months. Changing the type of dopant in the doped layers produced similar characterization results. However, the depth of the modulation fringes was different. This phenomenon indicates that dopant types play a role in determining the nonlinearity profile of the poled multilayer structures.

A hypothesis was presented using a MATLAB model to apprehend the experimental observations. The samples that produce modulation fringes may be possessing two thin nonlinear regions that interact to cause the destructive and constructive interferences. One of the layers could be located at the multilayer stack while the other one on the opposite side of the sample. The samples that do not show modulation fringes probably has only a thin nonlinear region. The width of this layer should be 
smaller than that of poled bulk silica.

\subsection{Recommendations of Future Work}

The findings of this research project unveiled some key features of the induced nonlinearity in multilayer silica structures. The hypothesis proposed in this thesis is subject to debate and verification. The following investigations will be valuable steps forward on this topic:

Characterization Methods The SHG microscopy method is commonly used to obtain a direct visualization of nonlinearity profiles in poled glass [13] [50]. In this technique, the laser beam is scanned across the side of the sample, so that the fundamental wave travels along the nonlinear region and normal to the direction of the poling field. The corresponding SH signal will show rise at the locations of the nonlinear regions only, thus helping to determine the exact locations and profile of the nonlinearity.

The Maker fringe characterization technique has been developed into several variations over the years. Prism- [51], sphere- [52], cylinder- [53] [54] assisted Maker fringe measurement are among the many enhancements available today. Implementing these improved techniques can help reveal attributes of the induced nonlinearity that would otherwise remain hidden. One advantage of the Maker fringe characterization method is that it is non-destructive.

Accelerated aging study Annealing of the multilayer structures for an accelerated aging study will rearrange the glass structure, stabilize the refractive index, decrease the film defects and relieve film stresses. Upon poling and characterization of the annealed samples, it would be possible to discern the true contribution of multilayer stack in breaking the symmetry of the silica glass to allow even-ordered nonlinearity.

Modifications of the multilayer structures Germanium doped multilayer structures were the primary focus of this research project. The brief study with the phosphorus doped samples indicated that dopant types affect induced nonlinearity in the samples. Therefore, it maybe worthwhile to examine the effect of different dopants types on the induced nonlinearity. 
The dopant concentration in the doped silica layers is another important parameter. It is reasonable to expect that a concentration threshold exists, below which the effect of the doped layers weakens. Optimizing for dopant concentration can be advantageous in refining the model of the multilayer structures.

Some other parameters that possibly play a crucial role in inducing nonlinearity in the multilayer structures are the individual layer width, the total width of all the layers, the total number of layers, the thickness of the silica substrate and type of silica substrate (e.g. fused silica and lead glass).

Poling methods In this research project, the samples were poled using the corona poling method. Thermal poling is another way commonly used to pole glasses. The parallel plate electrodes in thermal poling create a different electric field distribution over the sample than the the tungsten needle (in corona poling). This difference can cause different nonlinearity profiles in the poled samples. Another aspect to consider is that poling methods (mentioned in 1.1.1) were developed mainly to induce nonlinearity in structures such as bulk silica substrates and optical fiber. It is likely that the poling conditions of the existing methods are not optimum for the multilayer structures. It is imperative to research on the optimal poling conditions, such as poling voltage, duration, temperature, and electrode configuration, dedicated to multilayer silica structures. This study can also give insight into the poling effect at individual layers in the structure.

Silica glass is an indispensable material in photonic applications. For several decades, research efforts saw little advancement in inducing nonlinearity in the material so that it would be suitable for standard waveguide- and fiber-based applications. The model of multilayer structure shows the prospect of successfully implementing silica glass for practical applications involving even-ordered nonlinearities. This research project identified some fundamental aspects of the induced nonlinearity in the multilayer structures. These findings are meant to trigger new research ideas and contribute to the tool box for engineering silica glass to build active nonlinear devices. 


\section{List of References}

[1] E. Udd and W. B. Spillman Jr, Fiber optic sensors: an introduction for engineers and scientists. John Wiley \& Sons, 2011.

[2] M. Fiorentino, P. L. Voss, J. E. Sharping, and P. Kumar, "All-fiber photonpair source for quantum communications," IEEE Photonics Technology Letters, vol. 14, no. 7, pp. 983-985, 2002.

[3] I. Rau and F. Kajzar, "Second harmonic generation technique and its applications," Nonlinear Optics and Quantum Optics, vol. 38, p. 99, 2008.

[4] L. Arizmendi, "Photonic applications of lithium niobate crystals," physica status solidi (a), vol. 201, no. 2, pp. 253-283, 2004.

[5] U. Österberg and W. Margulis, "Dye laser pumped by nd: Yag laser pulses frequency doubled in a glass optical fiber," Optics letters, vol. 11, no. 8, pp. 516518,1986 .

[6] R. Myers, N. Mukherjee, and S. R. Brueck, "Large second-order nonlinearity in poled fused silica," Optics Letters, vol. 16, no. 22, pp. 1732-1734, 1991.

[7] H. An and S. Fleming, "Characterization of a second-order nonlinear layer profile in thermally poled optical fibers with second-harmonic microscopy," Optics letters, vol. 30, no. 8, pp. 866-868, 2005.

[8] A. Kudlinski, Y. Quiquempois, and G. Martinelli, "Room temperature aging of the $\chi(2)$ susceptibility induced in silica glasses by thermal poling," Applied Physics Letters, vol. 86, no. 16, p. 161909, 2005.

[9] A. Okada, K. Ishii, K. Mito, and K. Sasaki, "Phase-matched second-harmonic generation in novel corona poled glass waveguides," Applied Physics Letters, vol. 60, no. 23, pp. 2853-2855, 1992.

[10] P. Blazkiewicz, W. Xu, D. Wong, J. Canning, M. Åsland, and G. Town, "Carbon dioxide laser-assisted poling of silicate-based optical fibers," Optics letters, vol. 25, no. 4, pp. 200-202, 2000.

[11] T. Fujiwara, D. Wong, Y. Zhao, S. Fleming, S. Poole, and M. Sceats, "Electrooptic modulation in germanosilicate fibre with uv-excited poling," Electronics Letters, vol. 31, no. 7, pp. 573-575, 1995. 
[12] C. Corbari, P. G. Kazansky, S. A. Slattery, and D. N. Nikogosyan, "Ultraviolet poling of pure fused silica by high-intensity femtosecond radiation," Applied Physics Letters, vol. 86, no. 7, 2005.

[13] P. Kazansky, A. Kamal, and P. S. J. Russell, "High second-order nonlinearities induced in lead silicate glass by electron-beam irradiation," Optics letters, vol. 18, no. 9, pp. 693-695, 1993.

[14] L. J. Henry, B. V. McGrath, T. G. Alley, and J. J. Kester, "Optical nonlinearity in fused silica by proton implantation," JOSA B, vol. 13, no. 5, pp. 827-836, 1996.

[15] A. Ozcan, M. Digonnet, G. Kino, F. Ay, and A. Aydinli, "Characterization of thermally poled germanosilicate thin films," Optics Express, vol. 12, no. 20, pp. 4698-4708, 2004.

[16] A. C. Liu, M. J. Digonnet, and G. S. Kino, "Electro-optic phase modulation in a silica channel waveguide," Optics letters, vol. 19, no. 7, pp. 466-468, 1994.

[17] A. Kudlinski, G. Martinelli, and Y. Quiquempois, "Dynamics of the secondorder nonlinearity induced in suprasil glass thermally poled with continuous and alternating fields," Journal of Applied Physics, vol. 103, no. 6, p. 063109, 2008.

[18] Y. Quiquempois, N. Godbout, and S. Lacroix, "Model of charge migration during thermal poling in silica glasses: Evidence of a voltage threshold for the onset of a second-order nonlinearity," Physical Review A, vol. 65, no. 4, p. 043816, 2002.

[19] P. Kazansky and P. S. J. Russel, "Thermally poled glass: frozen-in electric field or oriented dipoles?," Optics Communications, vol. 110, no. 5-6, pp. 611-614, 1994.

[20] T. G. Alley, S. Brueck, and R. A. Myers, "Space charge dynamics in thermally poled fused silica," Journal of non-crystalline solids, vol. 242, no. 2, pp. 165-176, 1998.

[21] M. Dussauze, V. Rodriguez, A. Lipovskii, M. Petrov, C. Smith, K. Richardson, T. Cardinal, E. Fargin, and E. Kamitsos, "How does thermal poling affect the structure of soda-lime glass?," The Journal of Physical Chemistry C, vol. 114, no. 29, pp. 12754-12759, 2010.

[22] P. Kazansky, A. Smith, P. S. J. Russell, G. Yang, and G. Sessler, "Thermally poled silica glass: Laser induced pressure pulse probe of charge distribution," Applied Physics Letters, vol. 68, no. 2, pp. 269-271, 1996.

[23] D. Carlson, "Ion depletion of glass at a blocking anode: I, theory and experimental results for alkali silicate glasses," Journal of the American Ceramic Society, vol. 57, no. 7, pp. 291-294, 1974.

[24] U. K. Krieger and W. A. Lanford, "Field assisted transport of na+ ions, ca2+ ions and electrons in commercial soda-lime glass i: Experimental," Journal of Non-Crystalline Solids, vol. 102, no. 1-3, pp. 50-61, 1988. 
[25] N. Mukherjee, R. Myers, and S. Brueck, "Dynamics of second-harmonic generation in fused silica," JOSA B, vol. 11, no. 4, pp. 665-669, 1994.

[26] F. Garcia, I. Carvalho, E. Hering, W. Margulis, and B. Lesche, "Inducing a large second-order optical nonlinearity in soft glasses by poling," Applied physics letters, vol. 72, no. 25, pp. 3252-3254, 1998.

[27] T. G. Alley and S. Brueck, "Visualization of the nonlinear optical space-charge region of bulk thermally poled fused-silica glass," Optics letters, vol. 23, no. 15, pp. 1170-1172, 1998.

[28] H. G. de Chatellus, S. Montant, and E. Freysz, "Nondestructive method for characterization of the second-order nonlinear profile and charge distribution in thermally poled fused silica," Optics letters, vol. 25, no. 23, pp. 1723-1725, 2000.

[29] E. Y. Zhu, L. Qian, L. Helt, M. Liscidini, J. Sipe, C. Corbari, A. Canagasabey, M. Ibsen, and P. G. Kazansky, "Measurement of $\chi(2)$ symmetry in a poled fiber," Optics letters, vol. 35, no. 10, pp. 1530-1532, 2010.

[30] R. L. Sutherland, Handbook of nonlinear optics. CRC press, 2003.

[31] P. Maker, R. Terhune, M. Nisenoff, and C. Savage, "Effects of dispersion and focusing on the production of optical harmonics," Physical review letters, vol. 8, no. 1, p. 21, 1962.

[32] J. Jerphagnon and S. Kurtz, "Maker fringes: a detailed comparison of theory and experiment for isotropic and uniaxial crystals," Journal of Applied Physics, vol. 41, no. 4, pp. 1667-1681, 1970.

[33] R. W. Boyd, Nonlinear optics. Academic press, 2003.

[34] B. E. Saleh, M. C. Teich, and B. R. Masters, "Fundamentals of photonics," Journal of Biomedical Optics, vol. 13, no. 4, p. 049901, 2008.

[35] J. Arentoft, M. Kristensen, K. Pedersen, S. Bozhevolnyi, and P. Shi, "Large second-harmonic generation in thermally poled silica waveguides," in Optical Fiber Communication Conference and Exhibit, 2001. OFC 2001, vol. 1, pp. MC6MC6, IEEE, 2001.

[36] Y. Luo, A. Biswas, A. Frauenglass, and S. Brueck, "Large second-harmonic signal in thermally poled lead glass-silica waveguides," Applied physics letters, vol. 84, no. 24, pp. 4935-4937, 2004.

[37] H. An and S. Fleming, "Hindering effect of the core-cladding interface on the progression of the second-order nonlinearity layer in thermally poled optical fibers," Applied Physics Letters, vol. 87, no. 10, p. 101108, 2005.

[38] H. An and S. Fleming, "Time evolution of the second-order nonlinearity layer in thermally poled optical fiber," Applied physics letters, vol. 89, no. 23, p. 231105, 2006 . 
[39] D. Faccio, A. Busacca, D. Harwood, G. Bonfrate, V. Pruneri, and P. Kazansky, "Effect of core-cladding interface on thermal poling of germano-silicate optical waveguides," Optics communications, vol. 196, no. 1, pp. 187-190, 2001.

[40] H. An and S. Fleming, "Characterisation of thermally poled multilayered silicate thin films," in Opto-Electronics and Communications Conference, 2008 and the 2008 Australian Conference on Optical Fibre Technology. OECC/ACOFT 2008. Joint conference of the, pp. 1-2, IEEE, 2008.

[41] K. Yadav, C. Smelser, S. Jacob, C. Blanchetiere, C. Callender, and J. Albert, "Simultaneous corona poling of multiple glass layers for enhanced effective secondorder optical nonlinearities," Applied Physics Letters, vol. 99, no. 3, p. 031109, 2011.

[42] K. Yadav, C. Callender, C. Smelser, C. Ledderhof, C. Blanchetiere, S. Jacob, and J. Albert, "Giant enhancement of the second harmonic generation efficiency in poled multilayered silica glass structures," Optics express, vol. 19, no. 27, pp. 26975-26983, 2011.

[43] W. Angerer, N. Yang, A. Yodh, M. Khan, and C. Sun, "Ultrafast secondharmonic generation spectroscopy of gan thin films on sapphire," Physical Review $B$, vol. 59, no. 4, p. 2932, 1999.

[44] N. P. Bansal and R. H. Doremus, Handbook of glass properties. Elsevier, 2013.

[45] D. L. Griscom, "Trapped-electron centers in pure and doped glassy silica: A review and synthesis," Journal of Non-Crystalline Solids, vol. 357, no. 8, pp. 19451962, 2011.

[46] F. C. Garcia, L. Vogelaar, and R. Kashyap, "Poling of a channel waveguide," Optics express, vol. 11, no. 23, pp. 3041-3047, 2003.

[47] J. Eldridge and D. Kerr, "Sodium ion drift through phosphosilicate glass-sio2 films," Journal of the Electrochemical Society, vol. 118, no. 6, pp. 986-991, 1971.

[48] P. Nasr, "A theoretical study of maker fringe measurements in poled multi-layer silica structures focusing on the impact of layer quantity and spacing," Master's thesis, Carleton University Ottawa, 2015.

[49] I. Malitson, "Interspecimen comparison of the refractive index of fused silica," Josa, vol. 55, no. 10, pp. 1205-1209, 1965.

[50] H. An, S. Fleming, and G. Cox, "Visualization of second-order nonlinear layer in thermally poled fused silica glass," Applied physics letters, vol. 85, no. 24, pp. 5819-5821, 2004.

[51] D. Pureur, A. Liu, M. Digonnet, and G. Kino, "Absolute measurement of the second-order nonlinearity profile in poled silica," Optics letters, vol. 23, no. 8, pp. 588-590, 1998. 
[52] Y. Quiquempois, G. Martinelli, F. Valentin, P. Bernage, P. Nilay, and M. Douay, "Improved method for measuring second-order non-linearity profile in poled silica," in Bragg Gratings, Photosensitivity, and Poling in Glass Waveguides, p. DB9, Optical Society of America, 1999.

[53] A. Ozcan, M. Digonnet, and G. Kino, "Cylinder-assisted maker-fringe technique," Electronics Letters, vol. 39, no. 25, p. 1, 2003.

[54] A. Ozcan, M. Digonnet, and G. Kino, "Iterative processing of second-order optical nonlinearity depth profiles," Optics express, vol. 12, no. 15, pp. 3367-3376, 2004 . 